\title{
Summary of Propagation Cases of the Second AIAA Sonic Boom Prediction Workshop
}

\author{
Sriram K. Rallabhandi* and Alexandra Loubeau ${ }^{\dagger}$ \\ NASA Langley Research Center, Hampton, Virginia, 23681
}

\begin{abstract}
A summary is provided for the propagation portion of the second AIAA Sonic Boom Workshop held January 8, 2017 in conjunction with the AIAA SciTech 2017 conference. Near-field pressure waveforms for two cases were supplied and ground signatures at multiple azimuthal angles as well as their corresponding loudness metrics were requested from 10 participants, representing 3 countries. Each case had some required runs, as well as some optional runs. The required cases included atmospheric profiles with measured data including winds, using Radiosonde balloon data at multiple geographically spread locations, while the optional cases included temperature and pressure profiles from the US Standard atmosphere. The humidity profiles provided for the optional cases were taken from ANSI guidance, as the authors were unaware of an accepted standard at the time the cases were released to the participants. Participants provided ground signatures along with the requested data, including some loudness metrics using their best practices, which included lossy as well as lossless schemes. All the participants' submissions, for each case, are compared and discussed. Noise or loudness measures are calculated and detailed comparisons and statistical analyses are performed and presented. It has been observed that the variation in the loudness measures and spread between participants' submissions increased as the computation proceeded from under-track locations towards the lateral cut-off. Lessons learned during this workshop are discussed and recommendations are made for potential improvements and possible subsequent workshops as we collectively attempt to refine our analysis methods.
\end{abstract}

\section{Introduction and Motivation}

The Second American Institute of Aeronautics and Astronautics (AIAA) Sonic Boom Prediction Workshop (SBPW-2) was held on January 7-8, 2017 in Grapevine, Texas. Unlike the first workshop held in 2014, the first day was confined to near-field Computational Fluid Dynamics (CFD) predictions while the second day concentrated on atmospheric propagation predictions of ground signatures. This paper summarizes the propagation portion of the workshop. There were approximately 50 attendees from 7 nations and 10 participants in the propagation part of the workshop.

Many studies in the past 60 years have analyzed the numerical aspects of modeling and predicting sonic boom produced by an aircraft flying at supersonic speeds. Without delving into the specifics, the process involves computation of the flow-field close to the aircraft using CFD, extracting the pressure disturbance at an off-body location (called near-field), usually a few body-lengths below the aircraft, and finally using two-dimensional acoustic wave propagation techniques based on geometrical ray acoustic principles to predict the sonic boom at the ground. In this workshop, the near-field location is set at three body-lengths with the assumption that this distance is sufficiently far away from the aircraft so the 3D effects are fully resolved. These near-field pressure waveforms, extracted from CFD solutions, were supplied to the participants. The participants were requested to use their best practices and experience to predict ground signatures and their corresponding loudness values and ground intersection locations at several specified azimuthal angles, including lateral cut-offs under realistic atmospheric conditions with winds.

${ }^{*}$ Aerospace Engineer, Aeronautics Systems Analysis Branch, Systems Analysis and Concepts Directorate, MS 442, AIAA Associate Fellow

${ }^{\dagger}$ Research Aerospace Engineer, Structural Acoustics Branch, Research Directorate, MS 463 
The primary objectives of this workshop were a) To aid in the development of a supersonic aircraft noise certification standard, b) Verify analysis techniques within multiple codes across international teams, c) Understand modeling gaps and d) Improve awareness of sonic boom physics at realistic atmospheric conditions particularly at lateral cut-offs. The underlying motivations for this workshop were to a) Impartially compare propagated signatures from multiple teams/codes under standard and non-standard atmospheric conditions, b) Understand the state of current boom prediction methods across the international sonic boom community and c) Explore the effect of the atmosphere on the evolution of shaped sonic booms.

Since the goal of the sonic boom community is to understand how these sonic booms are perceived by humans and minimize their impact, statistical analysis of the loudness or noise metrics corresponding to the submissions is included in this summary. This succinctly quantifies the uncertainty of different propagation algorithms employed by international participants. The data and statistical analyses included in this summary are intended to contribute toward the discussion pertaining to overland supersonic flight by building a consensus in terms of analysis methods and modeling gaps and providing information to aid in developing a certification standard.

\section{I.A. Cases for propagation}

Before the propagation cases were down-selected, a survey was conducted to finalize the nature and number of cases to be included in the workshop. A diverse group of sonic boom experts from industry, academia and research organizations from across the world were contacted to complete the survey, resulting in eighteen distinct inputs. Based on the responses from the survey, it was decided to include two cases with some required and multiple optional runs. This decision was primarily made to test the propagation algorithms and loudness computation techniques without causing undue pressure on the participants to submit data corresponding to different atmospheric conditions for the same underlying aircraft concept or near-field pressure waveform. The main focus of comparison in this workshop was limited to atmospheric propagation a) at under-track and off-track azimuthal angles including near lateral cut-offs and b) under measured atmospheric profiles including winds as compared to a standard atmosphere. The two aircraft concepts considered are briefly described below.

\section{I.A.1. $\quad L M 1021$}

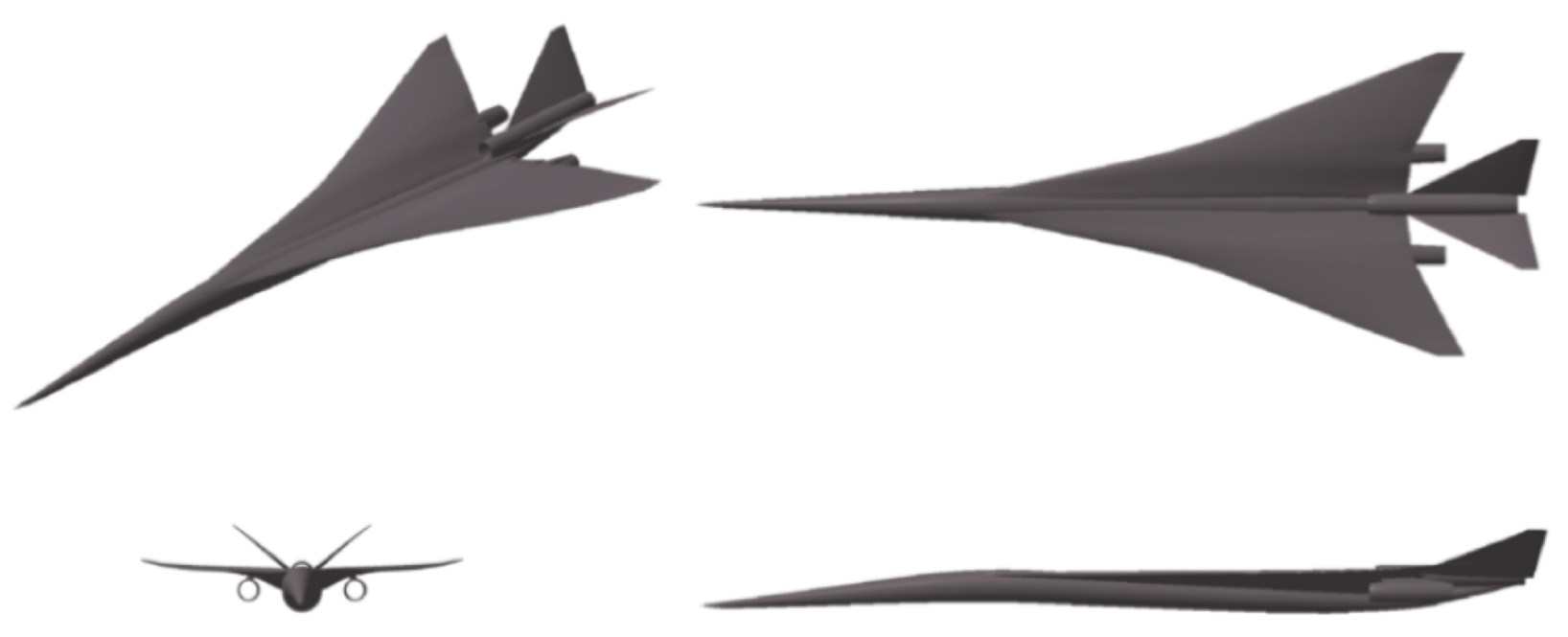

Figure 1. Perspective and three-view of LM1021 model.

In order to select cases for the propagation workshop, the first thought was to use the same concepts as were being used in the near-field CFD portion of the workshop. However, to test the propagation algorithms, the near-field pressures would have had to be supplied, and this would have defeated the purpose of the CFD portion of the workshop as it would have indicated the nature of the waveform, which was supposed to be obtained from the different participants without prior knowledge of expected results. To overcome this problem, the first case chosen was the optional case from the first Sonic Boom Prediction Workshop 


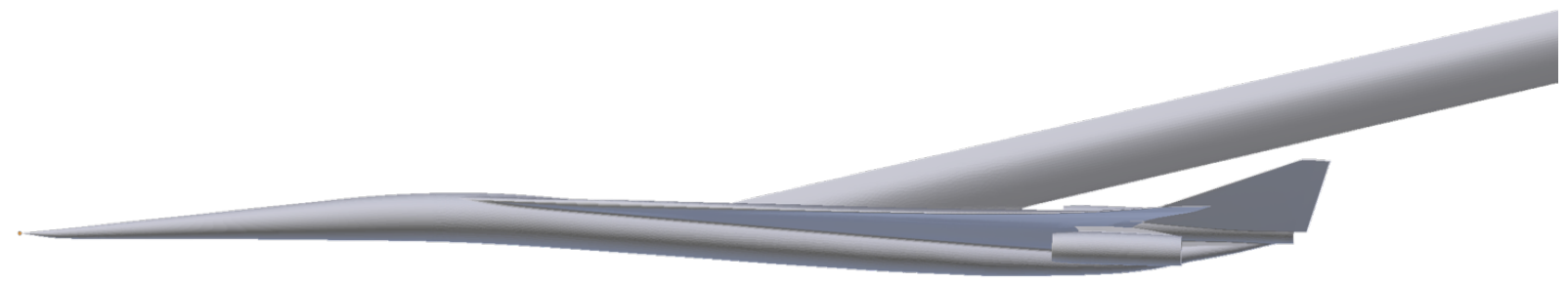

Figure 2. LM1021 wind-tunnel model with sting.

(SBPW1): ${ }^{1}$ LM1021 (See Fig. 1). With this choice, not only is the propagation based on an actual vehicle concept producing a non-N-wave signature on the ground, but also there is wind tunnel data as well as extensive documentation related to it stemming from the NASA High Speed (HS) Project's sponsored wind tunnel testing ${ }^{2}$ to validate low sonic boom designs. Although the wind tunnel model with sting (Fig. 2) used for CFD prediction in the first workshop worked well for near-field comparisons, using the waveform as-is during propagation to the ground resulted in the sting contribution dominating the effects from the actual aircraft. Therefore, the sting effect was numerically removed for the azimuthal angles shown in Fig. 3(a) and the resulting near-field waveforms used in the workshop are shown in Fig. 3(b).

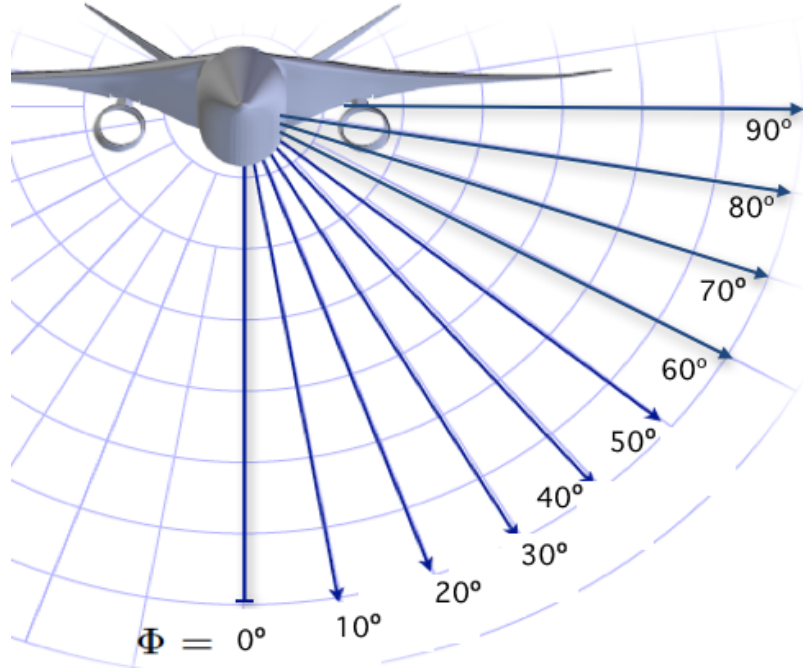

(a) LM1021 wind-tunnel model with sting.

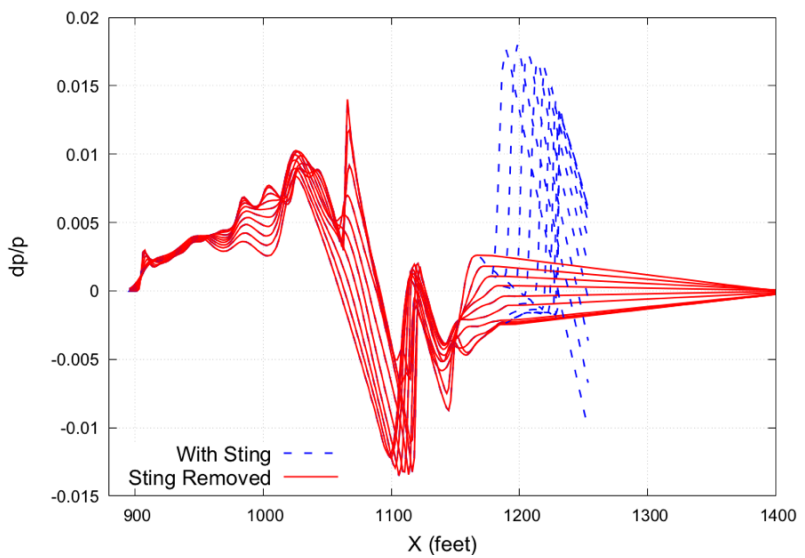

(b) LM1021 near-field pressures with and without sting.

Figure 3. LM1021 wind-tunnel correction.

\section{I.A.2. Axi-symmetric body of revolution (AXIBODY)}

For the second case, the desire was to use a low-boom concept. However, since there were no designs available from volunteers, the authors decided to generate a case inspired by a low-boom case, NASA's low-boom demonstrator Concept 25D, ${ }^{3}$ which was used in the CFD portion of the workshop. Since the near-field pressure waveform from 25D could not be used as-is because it would violate the near-field workshop blind test, an axi-symmetric body of revolution was inverse-designed using Cart3D ${ }^{4-6}$ framework to match 25D's near-field pressure waveform at an off-body distance of three body lengths below the vehicle. It should be noted that the final pressure waveform does not exactly match that from $25 \mathrm{D}$, as it was unnecessary to design for a perfect match and the objective was primarily to generate a case for the propagation workshop. Figure 4 depicts the pressure contours, adapted Cartesian mesh, and the near-field pressure waveform of the resulting body of revolution. 


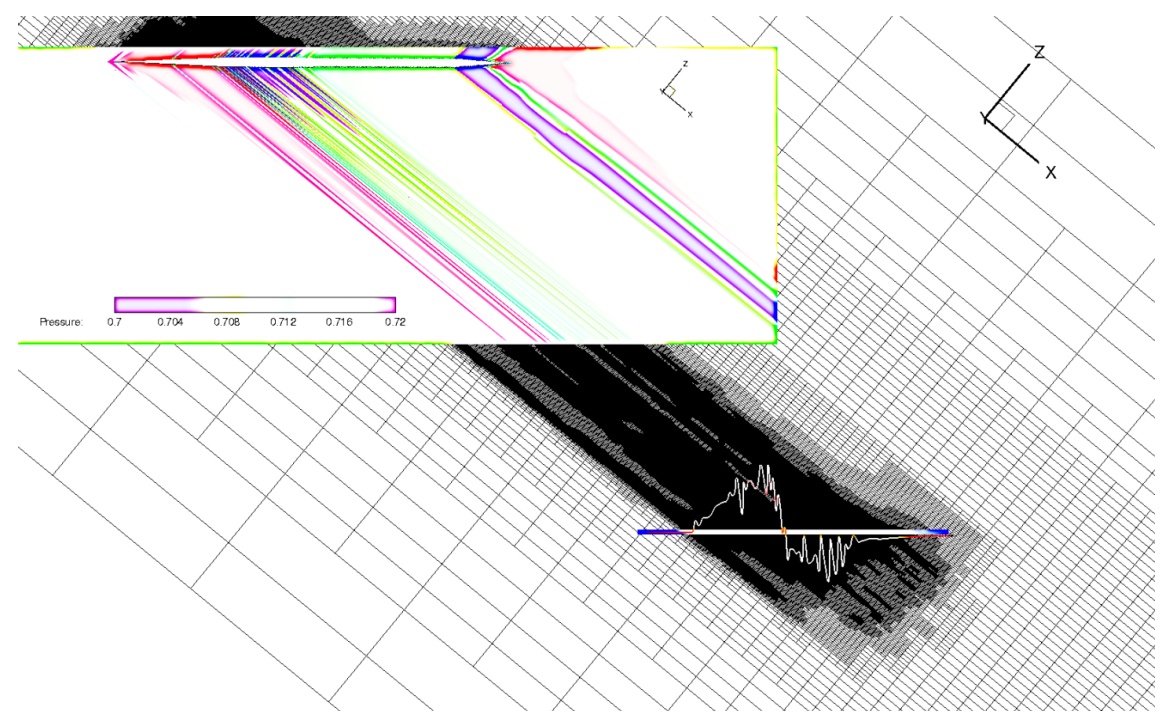

Figure 4. Axi-symmetric body, Cartesian mesh, near-field waveform and CFD flow-field for AXIBODY case.

\section{I.B. Selection of Atmospheric Conditions}

In addition to using multiple aircraft concepts for testing propagation algorithms, one of the objectives was also to test them under actual atmospheric conditions rather than using standard conditions as has been the norm in previous such comparisons. To this end, the authors looked at multiple sources and selected the Integrated Global Radiosonde Archive (IGRA) ${ }^{7}$ that consists of radiosonde balloon observations including temperature, pressure, dew point (a proxy for relative humidity), and winds at several active sites that are geographically separated. To narrow the scope of the problem, three sites in the United States were chosen in this study: a) Edwards Air Force Base, CA, b) Green Bay, WI, and c) Wallops, VA. These are not meant to be representative locations, but chosen randomly for convenience and to limit the computational runs in selecting atmospheric profiles to be supplied to the workshop participants.

For the LM1021 concept, all valid measured profiles at the above specified locations during a winter month (February, 2013) were selected for propagation to the ground using sBOOM. ${ }^{8}$ A similar exercise was carried out for the axi-symmetric body, except this time a summer month (August, 2012) was chosen. Figures 5(a) and 5(b) show the different cases run versus the Perceived Level (PL) in decibels (dB) corresponding to LM1021 and AXIBODY respectively. The highlighted circular markers represent the profiles chosen corresponding to the lowest and highest PL for each case. For both cases, the effect of atmosphere is significant, considering that loudness is measured on a logarithmic scale. Another important observation is that the variation due to the atmosphere is larger $(12 \mathrm{~dB})$ for the quieter AXIBODY compared to the louder LM1021 concept ( $8 \mathrm{~dB})$, indicating that the atmospheric effects and associated uncertainties become increasingly important as the underlying design produces shaped booms that keep reducing in terms of the PL metric.

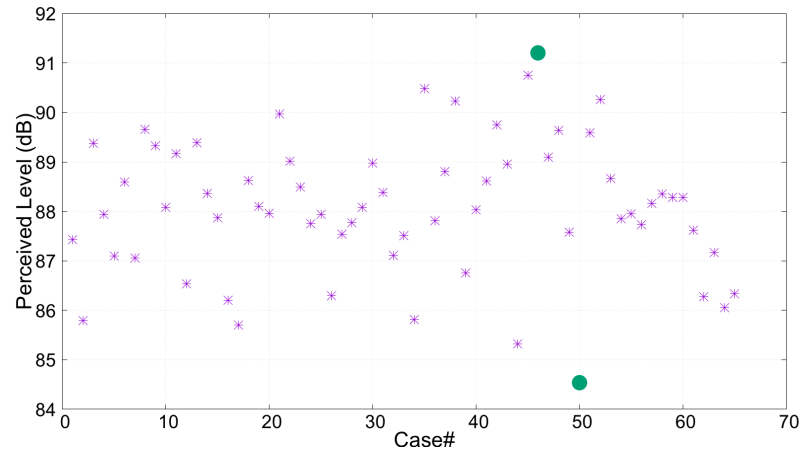

(a) Cases run in selecting LM1021 atmospheric profiles.

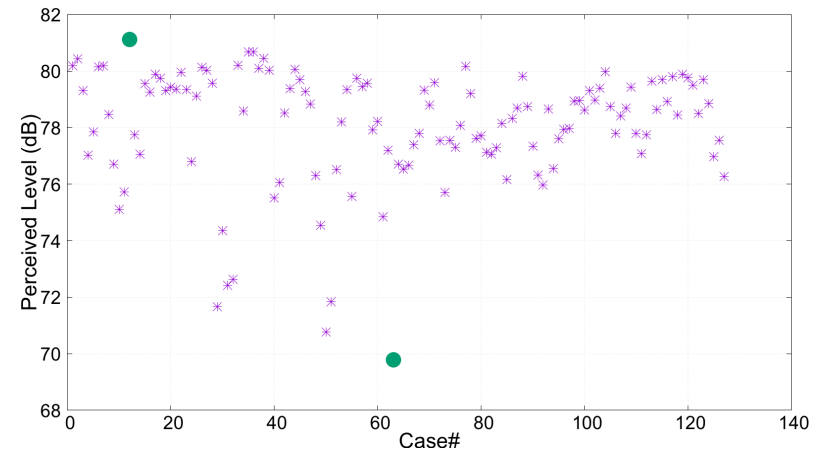

(b) Cases run in selecting AXIBODY atmospheric profiles.

Figure 5. Selection of Atmospheric Profiles. 
For the workshop, the atmospheric profiles corresponding to the lowest and highest PL, for each concept, are chosen. The regular practice is to run propagation at standard atmospheric reference conditions with no winds (temperature and pressure from Ref. ${ }^{9}$ ). The relative humidity for this case was chosen from ANSI S1.26, Annex C guidance. ${ }^{10}$ In addition, the ICAO Annex 16 Volume $1^{11}$ international standards and recommended practices for aircraft noise certification dictates reference conditions of $70 \%$ relative humidity at all altitudes with no wind. This $70 \%$ relative humidity was paired with the standard atmosphere temperature and pressure to create another atmospheric profile case. To study the impact of all these atmospheres on propagated ground signatures, all are included as either required or optional cases. A comparison of these is given in Figs. 6(a) and 6(b), where [T,WX,WY,RH] represent temperature, X-wind, Y-wind and relative humidity respectively. The numeric index values $([1,2,3,4])$ after each symbol represent the measured profiles' tuple [profile1, profile2, profile3, profile4] respectively. The details of the measured atmospheric profiles are listed in Table 1. While the selection of the active sites for measured atmospheric profiles is neither exhaustive nor representative, this is the first exercise in using non-standard profiles.

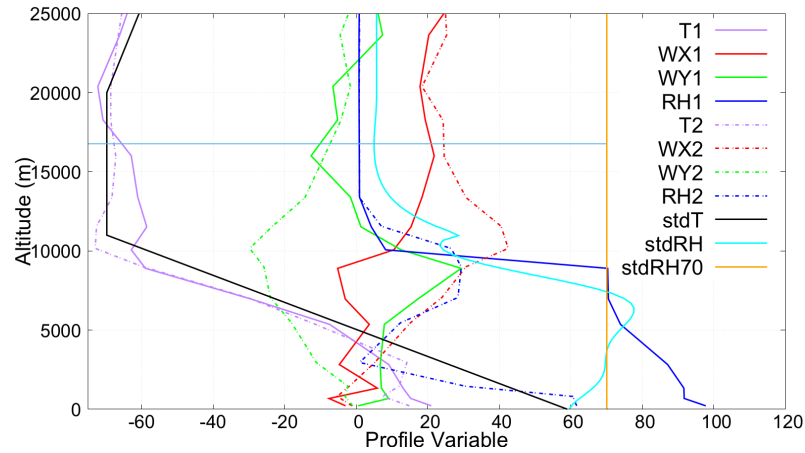

(a) Atmospheric profiles chosen for LM1021 cases.

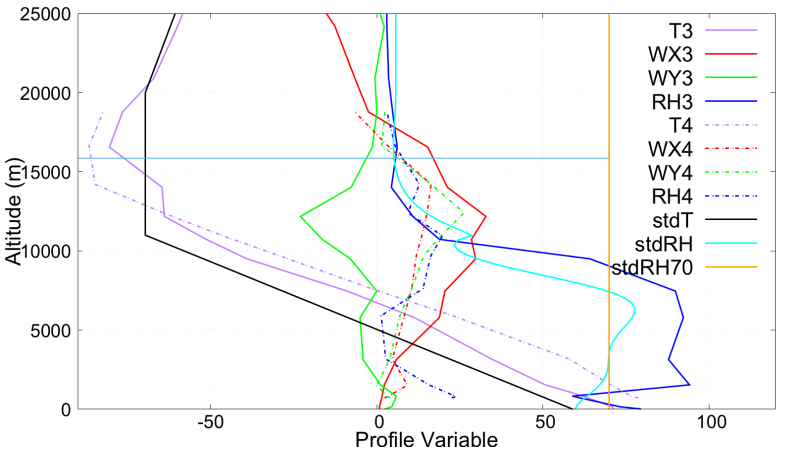

(b) Atmospheric profiles chosen for AXIBODY cases.

Figure 6. Chosen atmospheric profiles.

Table 1. Measured atmospheric profile index

\begin{tabular}{ccc}
\hline \hline Name & Case & Characteristic \\
\hline Profile1 (T1,RH1,WX1,WY1) & LM1021 & Highest PL among the cases considered \\
Profile2 (T2,RH2,WX2,WY2) & LM1021 & Lowest PL among the cases considered \\
Profile3 (T3,RH3,WX3,WY3) & AXIBODY & Highest PL among the cases considered \\
Profile4 (T4,RH4,WX4,WY4) & AXIBODY & Lowest PL among the cases considered \\
\hline \hline
\end{tabular}

\section{Evaluation Methods}

The participants submitted the required data such as ground signatures and corresponding optional data such as loudness/noise metrics. Even though comparing ground signatures can shed light on the results of multiple participants, the loudness measures offer advantages such as a) ease of comparison as they are scalars and therefore more amenable to the application of simpler statistical techniques, and b) being indicative of the community response/human perception. The data reduction needed was performed by the authors, not by the participants, in an attempt to apply these methods as uniformly as possible to all the submissions.

Loudness perception and annoyance are inherently subjective measures of a sonic boom experience. Many noise descriptors have been evaluated as loudness predictors in human experiments, for example see Leatherwood et al. ${ }^{12}$ The Mark VII Perceived Level (PL) of Stevens ${ }^{13}$ is adopted as a measure to compare submissions because it is correlated with loudness and annoyance in multiple experiments. ${ }^{12,14}$ The A-weighted Sound Exposure Level (ASEL) is also used because of its correlation ${ }^{12}$ with PL for outdoor signatures and analytical calculation procedure that can be used to plot the integrated loudness time history of the ground signature. The method of Shepherd and Sullivan ${ }^{15}$ is used to calculate PL and ASEL from ground signatures. 
Additional metrics are calculated to investigate the distribution of submission data. The six metrics considered have been shown to correlate well with multiple laboratory datasets of human annoyance to sonic booms in both outdoor and indoor environments; ${ }^{14}$ the metrics include PL, ASEL, BSEL, DSEL, ESEL, and ISBAP (Indoor Sonic Boom Annoyance Predictor ${ }^{14}$ ).

\section{Participants}

Overall, there were 10 participants, with one participant submitting two solutions using different methods. Out of the 11 submissions, 10 were based on lossy mechanisms, of which 7 were based on distinct implementations, while one submission was based on lossless weak-shock theory assumptions. Table 2 lists the details of each submission.

Table 2. Submission Index

\begin{tabular}{|c|c|c|}
\hline Participant Index & Propagation Type & Submissions \\
\hline $\mathrm{P} 1$ & Lossy Burgers & $\begin{array}{l}\text { Required \& optional cases } \\
\text { \& loudness metrics }\end{array}$ \\
\hline $\mathrm{P} 2$ & Lossy Burgers & $\begin{array}{l}\text { Required \& optional cases } \\
\text { \& loudness metrics }\end{array}$ \\
\hline P3 & Lossy Burgers & Required \& optional cases \\
\hline $\mathrm{P} 4$ & Lossy Burgers & $\begin{array}{c}\text { Required \& optional cases for all } \\
\text { supplied roll angles \& loudness metrics }\end{array}$ \\
\hline $\mathrm{P} 5$ & Lossy Burgers & $\begin{array}{l}\text { Required \& optional cases } \\
\text { \& loudness metrics }\end{array}$ \\
\hline P6 & $\begin{array}{c}\text { Multipole correction \& Lossy } \\
\text { Burgers }\end{array}$ & $\begin{array}{c}\text { Required cases } \\
\& \text { loudness metrics }\end{array}$ \\
\hline $\mathrm{P} 7$ & Lossy Burgers & $\begin{array}{c}\text { Required \& optional cases } \\
\text { \& loudness metrics }\end{array}$ \\
\hline $\mathrm{P} 8$ & Lossy Burgers & $\begin{array}{c}\text { Required \& optional cases for all } \\
\text { supplied roll angles \& loudness metrics }\end{array}$ \\
\hline P9 & Lossy Burgers & $\begin{array}{c}\text { Required cases } \\
\& \text { loudness metrics }\end{array}$ \\
\hline $\mathrm{P} 10$ & Lossy Burgers & $\begin{array}{c}\text { Required \& some optional cases } \\
\text { \& loudness metrics }\end{array}$ \\
\hline P11 & $\begin{array}{l}\text { Weak-shock (lossless) theory } \\
\text { with Taylor shock structure }\end{array}$ & $\begin{array}{c}\text { Required \& some optional cases } \\
\text { \& loudness metrics }\end{array}$ \\
\hline
\end{tabular}

\section{LM1021 Analysis}

This section provides details on the submissions received for the LM1021 concept. Figure 7 depicts the ground signatures of all submissions from the participants for all the atmospheric profiles directly beneath the flight track of the aircraft. The submissions are compared for the pressure magnitudes as well as duration. More detailed comparisons and statistics are presented later in the paper. For the standard profiles, all the submissions had almost the same duration, and except one submission from participant 4 (P4), all the other signatures are visually very similar. Specifying a constant relative humidity of $70 \%$ does not seem to disrupt the correlation between the submissions. However, for both non-standard profiles that have non-zero winds, larger variations in boom duration as well as shock magnitudes and locations are observed between submissions. This shows that the inclusion of winds in various numerical implementations leads to differences in the results. 


\section{IV.A. Ground Signatures and loudness metrics}

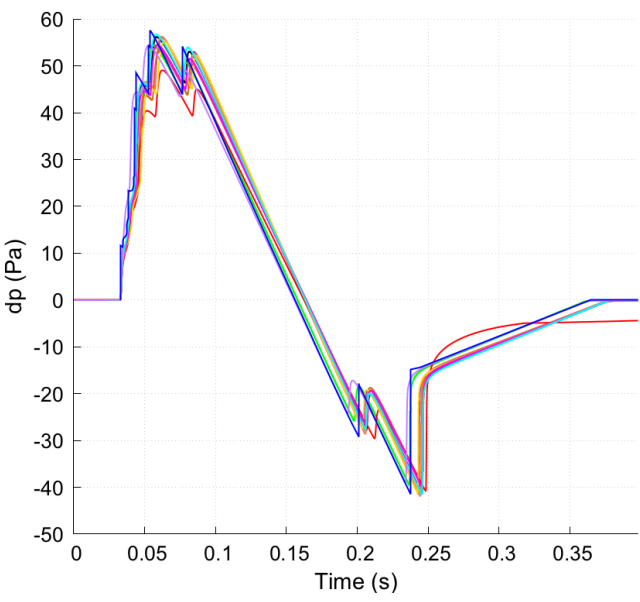

(a) Profile1 (Required).

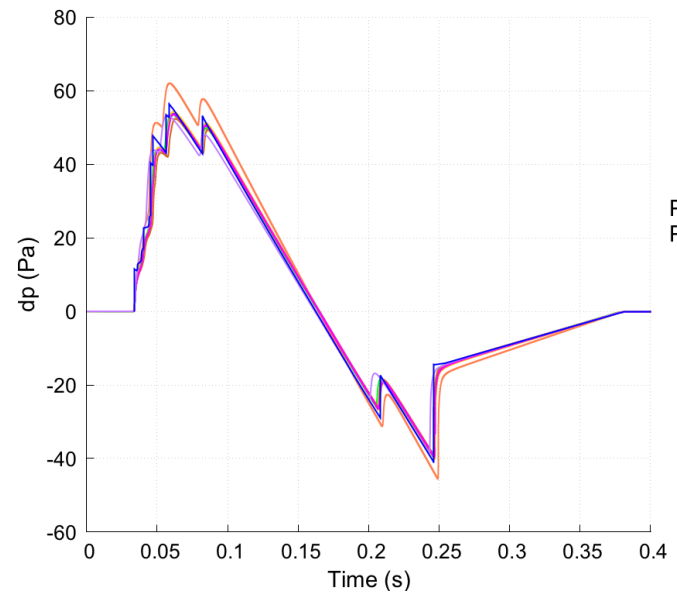

(c) Standard Profile (Optional).
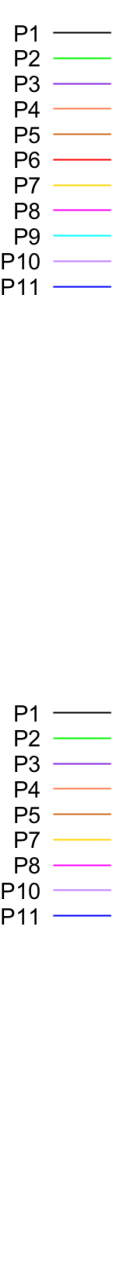

Figure 7. Ground Signatures for LM1021 at Roll Angle $=0^{\circ}$

As the analysis moves to off-track azimuthal angles, the discrepancies between the submissions seems to increase as seen in Figs. 8 and 9. For the standard profiles, specifying a constant relative humidity doesn't seem to effect the ground signatures from the same participant, but the differences between participants is larger compared to the under-track case, both in terms of signature duration and shock locations. These differences are amplified for the non-standard profiles (See figs. 8(a), 8(b), 9(a) and 9(b)). Since the standard profiles are modeled with no winds, the sonic boom carpet is laterally symmetric. However, for the cases with non-zero winds, the ground signatures and the associated boom metrics are asymmetric as seen by comparing ground signatures in figs. 8(a) and 8(b) with those from figs. 9(a) and 9(b).

Another difference seen is the predicted rise times and shock structure between different submissions. Fig. 10 shows a detailed view of the front and aft shock system corresponding to Profile1 for each of the submissions for under-track and $-30^{\circ}$ off-track location. The differences between the shock rise times are clearly apparent from this comparison. Since loudness metrics depend on the frequency spectrum of the ground signature, such differences can be expected to have a reasonably big impact.

The next set of plots depict the PL in decibels, across the carpet, for each of the atmospheric profiles considered. Fig. 11 depicts the loudness values submitted by participants while Fig. 12 depicts the loudness values calculated from the ground signature submissions. This exercise was meant not only to calibrate the current loudness calculation practices used by participants, but also evaluate the submissions uniformly based on the loudness calculation procedure ${ }^{15}$ available to the authors. Since the submission of loudness metrics was optional, not all participants submitted this information and those that submitted may not have submitted 


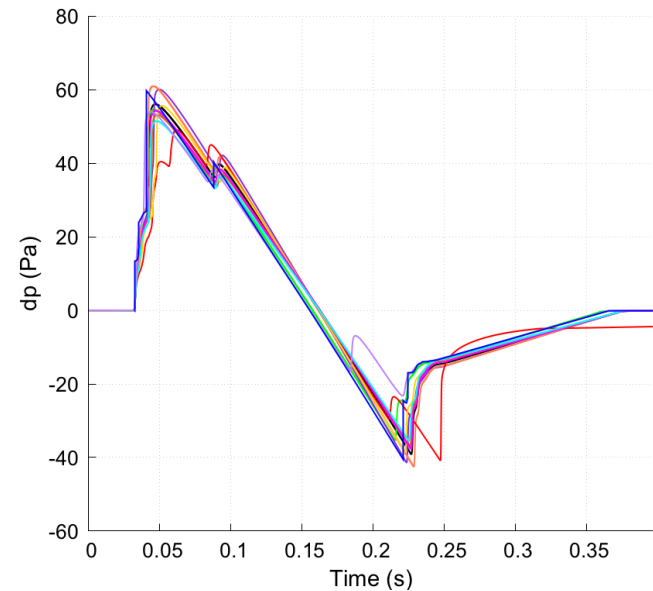

(a) Profile1 (Required).

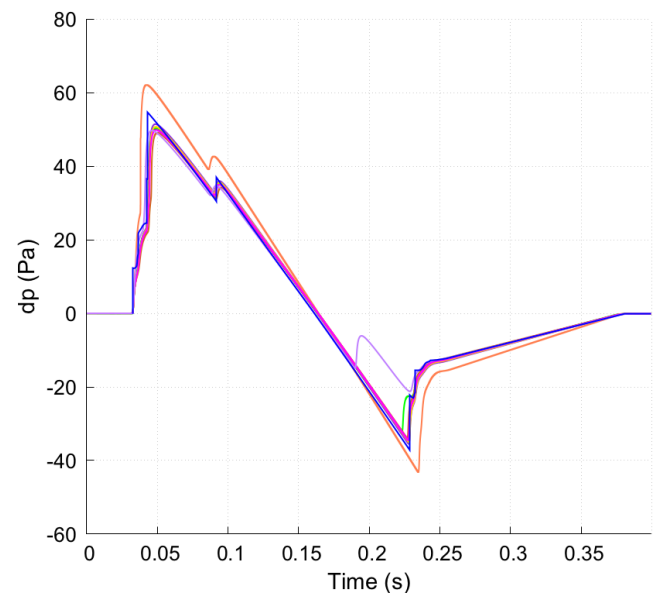

(c) Standard Profile (Optional).

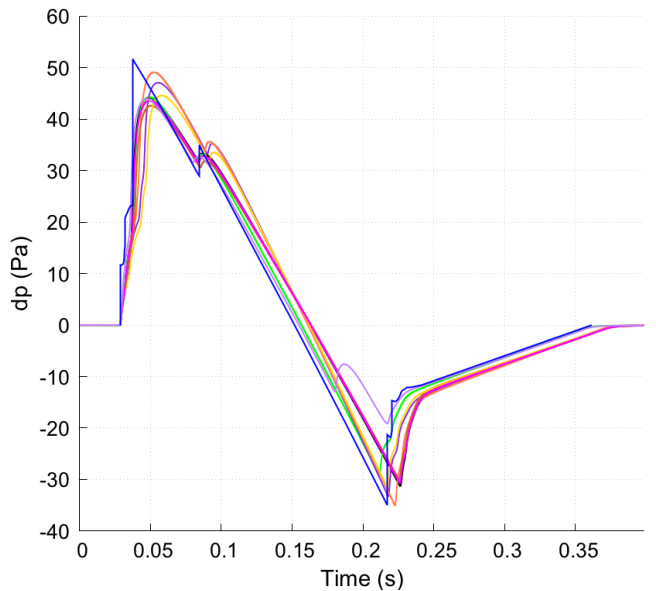

(b) Profile2 (Optional).

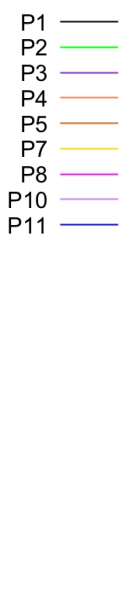

Figure 8. Ground Signatures for LM1021 at Roll Angle $=-30^{\circ}$

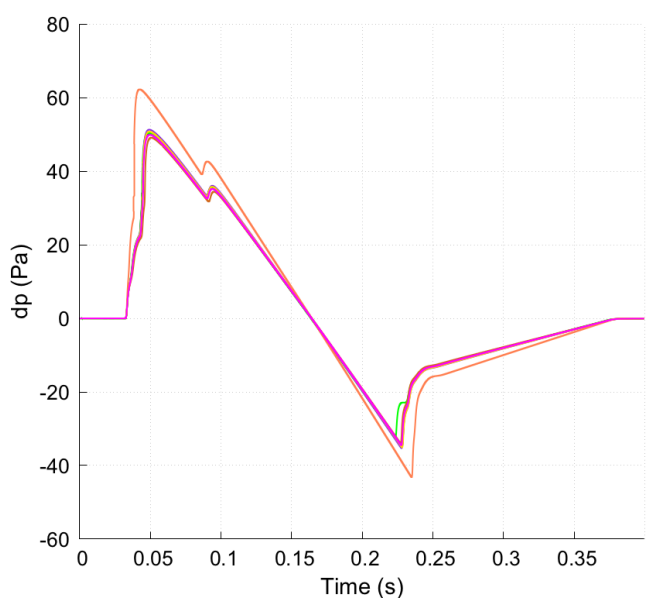

(d) Standard Profile w/ RH=70\% (Optional).

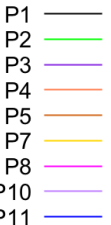

P2

P4

$\mathrm{P7}$

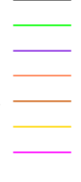

these at all azimuthal angles where the near-field pressure was supplied. There are certain submissions, such as P9 from Fig. 11(a), that seem to be the outlier in terms of loudness. However, referring back to Fig. 7 (a), the ground signature corresponding to this submission agrees quite well with the other submissions. Therefore, the loudness calculation scheme for that participant may be the cause for the discrepancy. Using a common model for loudness computations allows to separate the loudness calculation aspect from the atmospheric propagation and the corresponding results are plotted in Fig. 12. Now, the loudness values for P9 align well with the other participants. Submission from P11 resulted in much higher loudness because it does not include any losses during propagation and no additional assumptions were made during loudness calculation to correct for the shock rise times. The main observations from these figures are:

1. The loudness values are closely clustered near and under the flight track, while the spread increases as the analysis moves to lateral cut-off locations

2. The profiles are laterally symmetric for zero-wind standard profiles, and asymmetric for the profiles with winds, as expected

3. The submitted lateral cut-off angles are closely clustered, but the variation in the loudness metrics is the highest among all the azimuthal angles. The primary reason for such a large discrepancy is that ray grazing angles are fairly low near the carpet edges and even small discrepancies in the cut-off angles result in additional losses as the signal propagates over large distances at low altitudes, leading to larger differences 


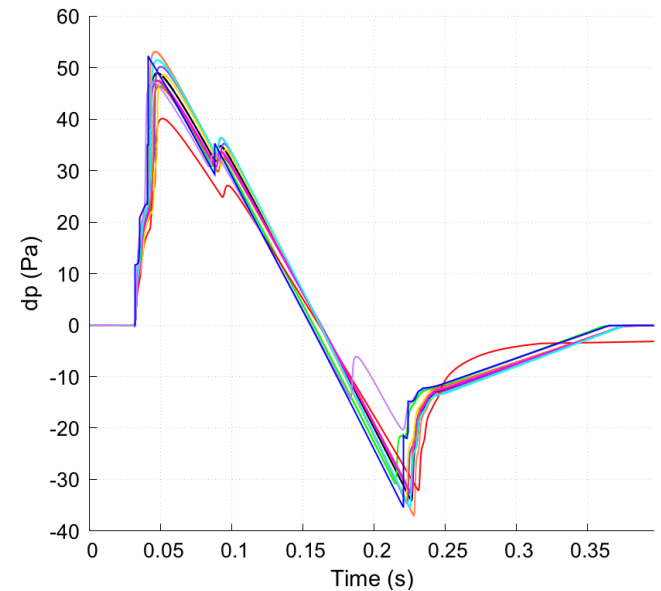

(a) Profile1 (Required).

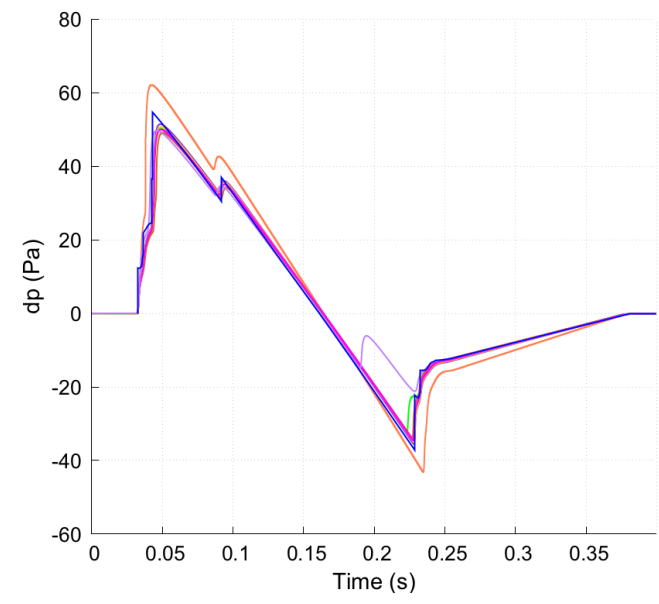

(c) Standard Profile (Optional).

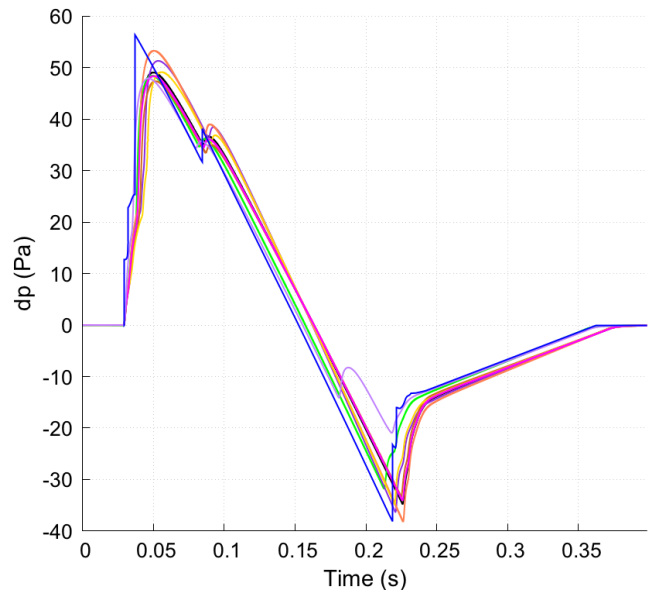

(b) Profile2 (Optional).

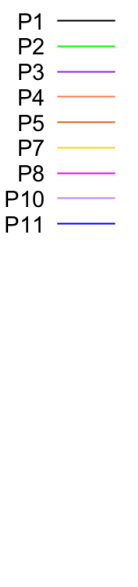

Figure 9. Ground Signatures for LM1021 at Roll Angle $=30^{\circ}$

4. Except for a couple of participants (P9 and P11), the calculated loudness values are fairly close to the submitted values. The calculated P11 loudness value does not correct the signature for a shock rise time and as expected the loudness values resulting from a loss-less propagation are expected to be higher. From that perspective, the only outlier as far as loudness computation is concerned is P9.

Figure 13 shows the PL convergence history as a function of the sampling frequency used during propagation. All submissions seem to suggest a certain minimum sampling frequency is needed to achieve convergence. This minimum is lower for some submissions compared to others, but data seems to suggest a value of greater than $50 \mathrm{kHz}$ for this configuration for under-track propagations. For off-track propagations (See figs. 14 and 15), the convergence data submitted, particularly for $\mathrm{P} 6$ and $\mathrm{P} 8$, is insufficient to conclude whether convergence has been achieved even at sampling frequencies beyond $200 \mathrm{kHz}$. Data submitted for P9 is also plotted, while acknowledging that the loudness values computed by this participant have been the outlier as shown in previous figures. The calculated convergence histories were not plotted because the participants were not required to submit their ground signatures corresponding to intermediate sampling frequencies.

The extent of the boom carpet as predicted by different participants is analyzed next using the range versus the PL plots for both the longitudinal and lateral ranges. Figure 16 depicts these for all the submissions that have the range data. For the measured profiles, the longitudinal (X) plots contain two branches, each corresponding to the port and star-board side of the aircraft flight track and the lateral asymmetry is visible on the PL versus Y plots as depicted in figs. 16(a) and 16(b). On the X plots, the lowest value of X corresponds to the under-track boom. For the zero wind standard profiles, owing to lateral symmetry of the boom carpet, these two branches lie on top of each other as shown in figs. 16(c) and 16(d). All these plots are vertically 


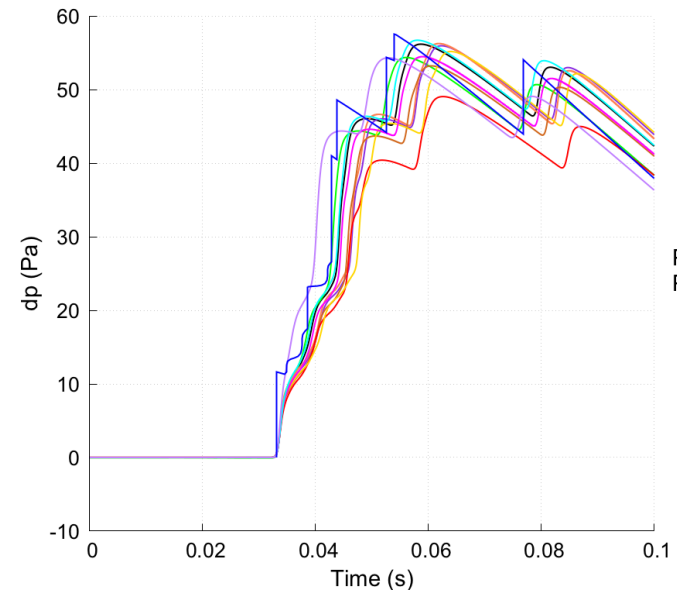

(a) Front shock system for Roll Angle $=0^{\circ}$.

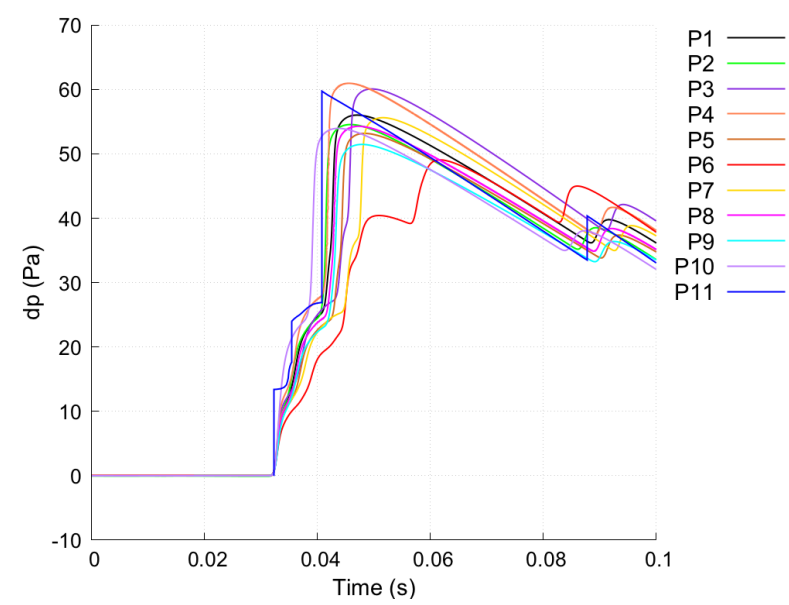

(c) Front shock system for Roll Angle $=-30^{\circ}$.

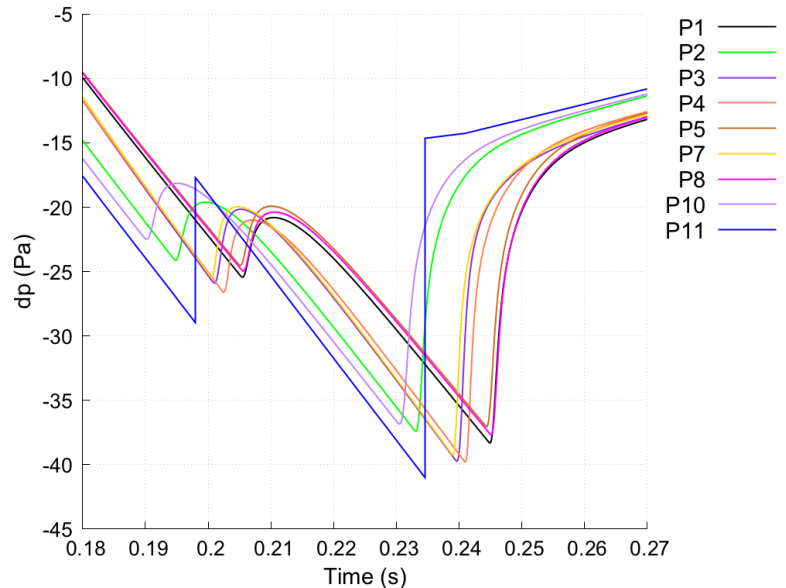

(b) Aft shock system for Roll Angle $=0^{\circ}$.

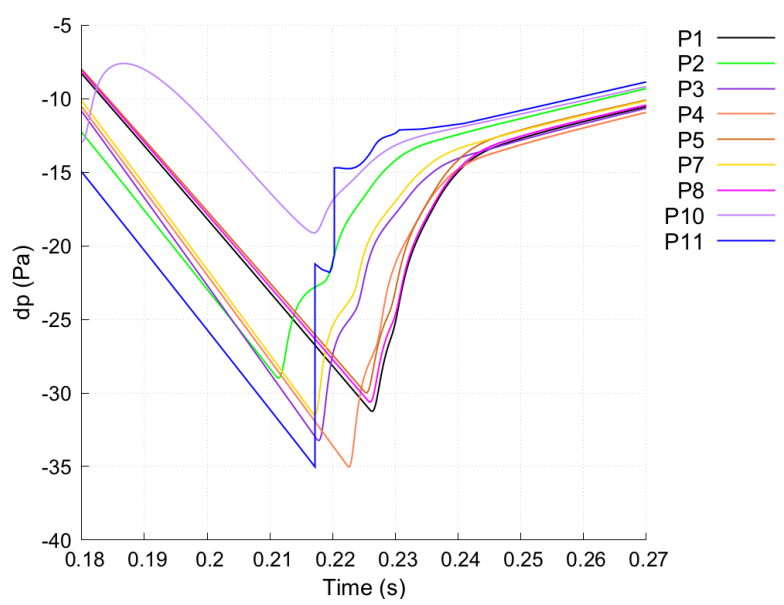

(d) Aft shock system for Roll Angle $=-30^{\circ}$.

Figure 10. Details of the front and aft shock systems of the ground signatures for LM1021

shifted with virtually no lateral shift. This shows that the ray paths are similar between the participants, the differences lie in implementation of propagation mechanisms and loudness calculation. In order to view the differences of just the propagation mechanisms without the uncertainty associated with loudness calculation, Fig. 17 compares the loudness versus ranges using a uniform loudness computation. The differences between submissions are seen to be primarily the result of underlying lossy propagation implementations, not the loudness calculation or the ray paths.

\section{IV.B. Spectral Analysis}

The spectral plots, especially those that dissect the calculation of PL reveal important information regarding the differences seen using this loudness measure. Figs. 18 and 19 depict the loudness spectra and Sound Pressure Level (SPL) spectra respectively corresponding to under-track boom signatures. The loudness spectra indicate the $1 / 3^{r d}$-octave frequency bands which are most important to the calculation of PL, which approximates the sensitivity of human hearing, while SPL spectra indicate the sound energy in different $1 / 3^{r d}$-octave frequency bands. From Fig. 18(a), P6 is a clear outlier, and as expected the computed PL is smaller than other participants' submissions (See Fig. 12(a)). As we move to optional cases, P4 becomes the outlier with predicted higher contributions at most frequencies. For the standard profile, P10 seems to have lesser contributions at higher frequencies, suggesting that perhaps the shocks are dissipated or smoother compared to other submissions. Similar trends are also seen in the SPL plots.

As the analysis moves to off-track locations, the spread between submissions widens as seen in Figs. 20 and 21. The differences are more pronounced at lower frequencies in the range [20-500] Hz, which suggests 


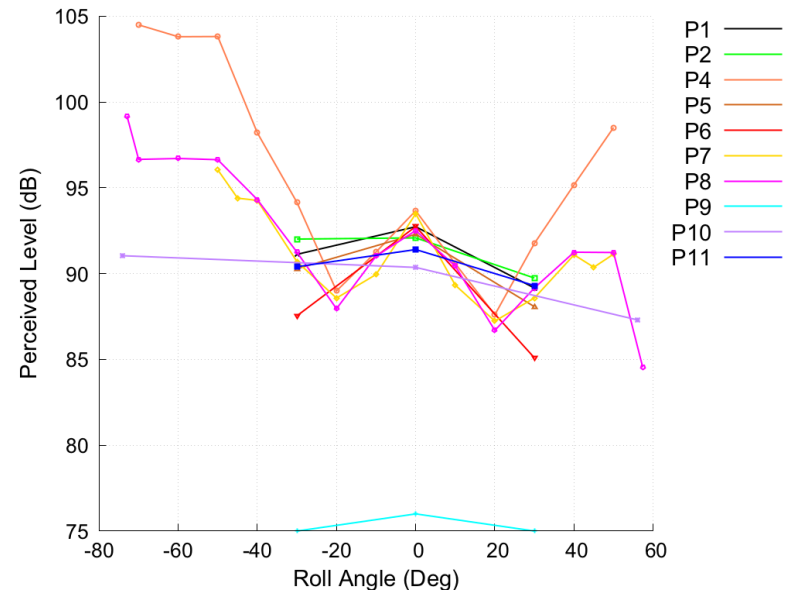

(a) Profile1 (Required).

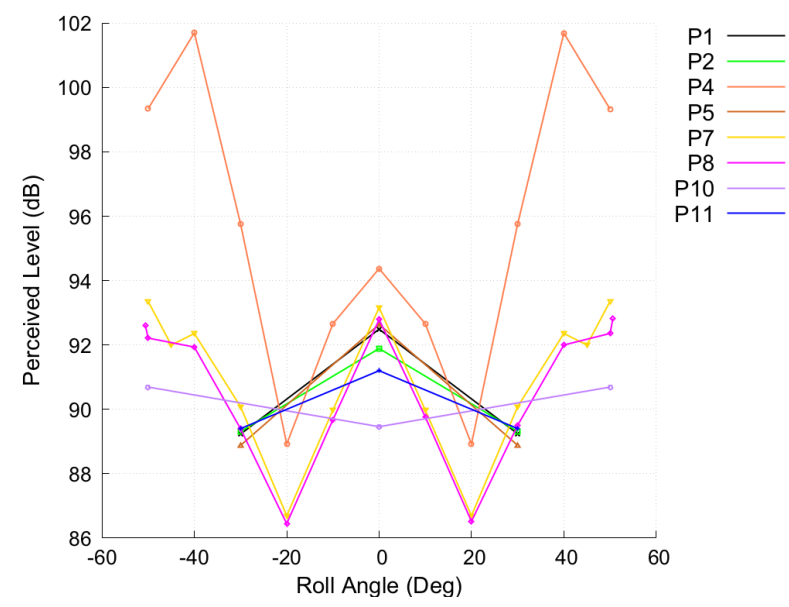

(c) Standard Profile (Optional).

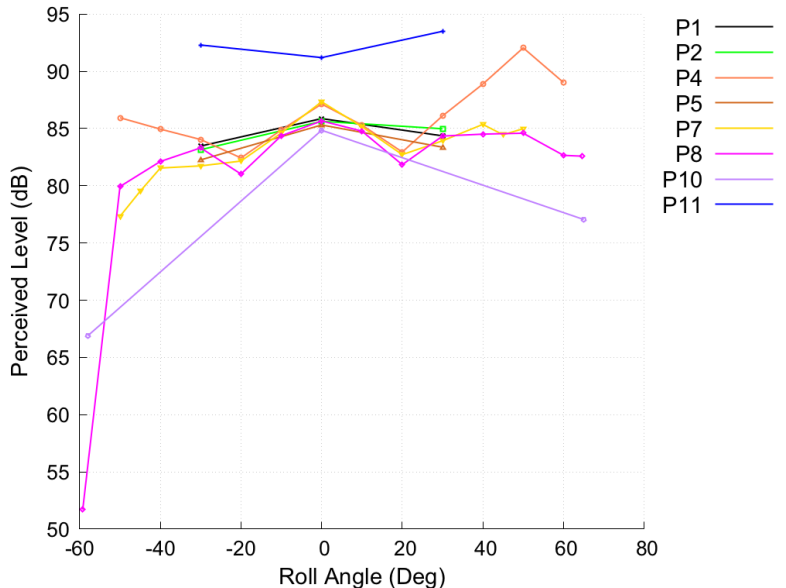

(b) Profile2 (Optional).

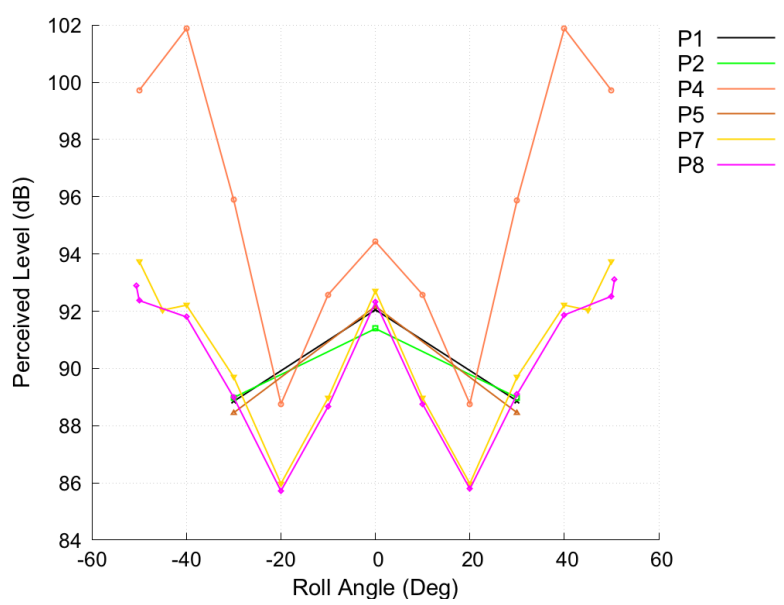

(d) Standard Profile w/ RH=70\% (Optional).

Figure 11. Submitted carpet loudness values for LM1021

that there are changes in the duration of the boom signal as well as macroscopic features or shape of the ground signature. The results for positive roll angles are similar to these plots, and are not shown here for the sake of brevity.

\section{IV.C. Statistical Analysis}

Whenever there is data assimilation from multiple schemes and participants, a visual way to depict the spread as either error bands or standard deviation is extremely helpful to gain insight into the differences. Fig. 22 shows the mean and one standard deviation of the spread for all the four atmospheric profiles at the under-track location. For these comparisons, the lossless propagation case (P11) is omitted as inclusion of that would amplify the differences. For both standard profiles, the spread across participants is small. The standard profile with fixed relative humidity exhibits the lowest spread, while allowing the humidity to vary introduces discrepancies, especially in the aft near the shocks. This shows that the inclusion of humidity is the cause for some differences between different implementations. Both the measured profiles have larger spreads compared to the standard profiles. This suggests that winds have a certain role in the discrepancies between the submissions.

As we move off-track (See figs. 23 and 24), the standard deviation bounds increase compared to the under-track locations. And the other trends, namely that humidity and winds affect the predicted signatures differently in different implementations, are confirmed. As expected, the standard deviations are laterally asymmetric for the measured profiles with non-zero winds. 


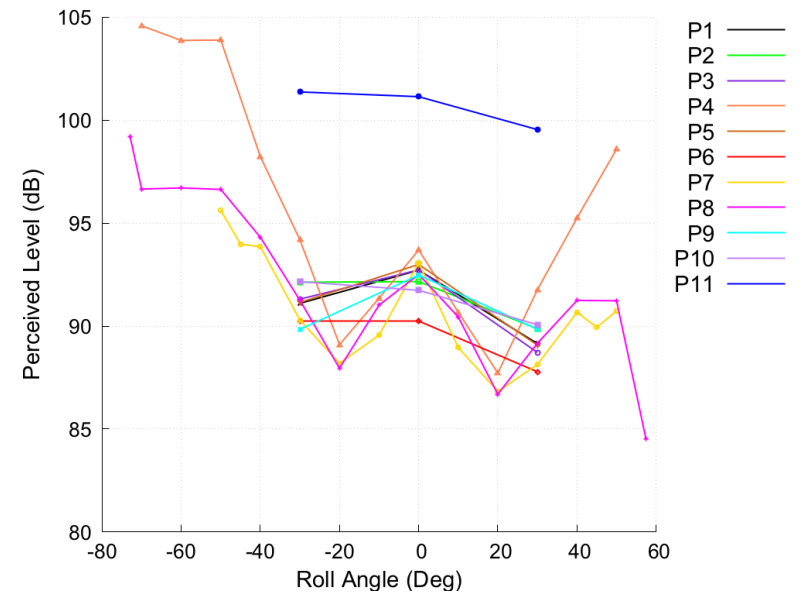

(a) Profile1 (Required).

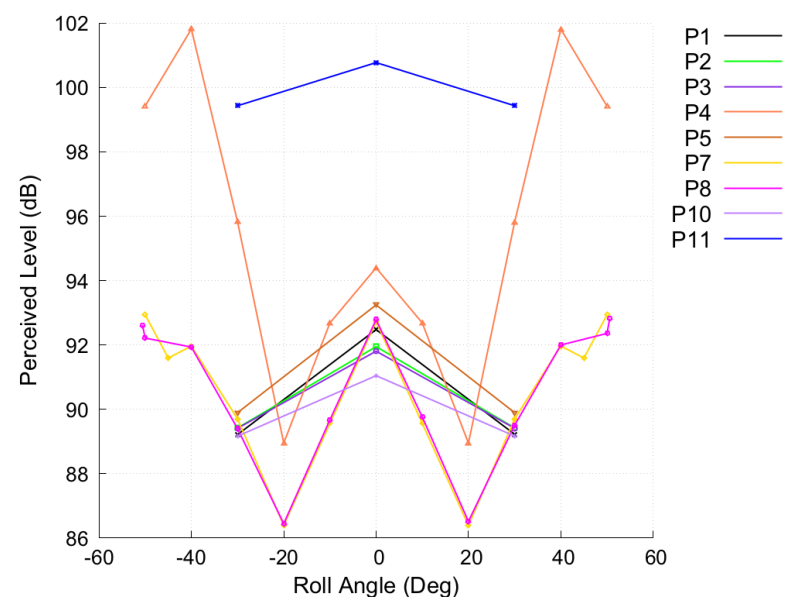

(c) Standard Profile (Optional).

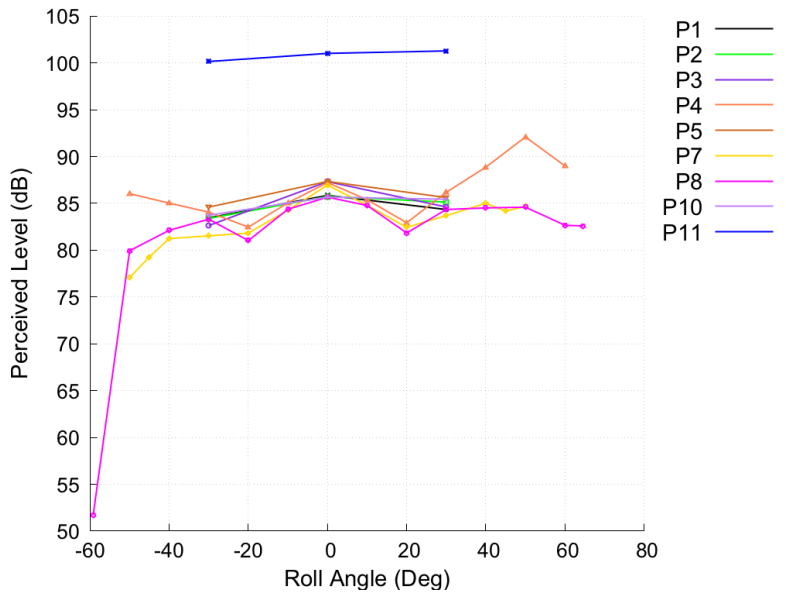

(b) Profile2 (Optional).

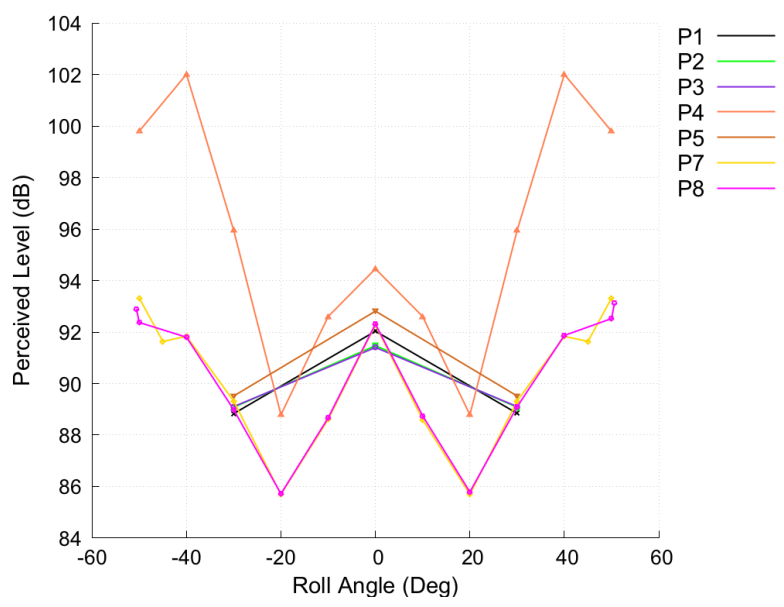

(d) Standard Profile w/ RH=70\% (Optional).

Figure 12. Calculated carpet loudness values for LM1021

The data are further examined through the use of violin plots, which show the distribution of data in addition to summary statistics such as a box plot indicating the median and the interquartile range. Violin plots for six noise metrics (PL, ASEL, BSEL, DSEL, ESEL, and ISBAP) are shown in Fig. 25; data from all submissions for all four atmospheric profiles at the angles of $-30,0$, and 30 degrees are included. The size of the distribution varies with each metric, with the range encompassing approximately $15 \mathrm{~dB}$ for both PL and ASEL. The extent and shape of these distributions indicates that the atmospheric profiles have a large effect on most of the metrics. The distribution is smallest for the DSEL metric, which may indicate that this metric is less sensitive to changes in the waveform due to atmospheric profile differences.

The data are then subdivided by atmospheric profile as shown in Fig. 26. The distributions encompass smaller ranges of less than $10 \mathrm{~dB}$, and it is shown that Profile 2 results in the lowest median values for all metrics. The distributions also appear more compact for Profile 2. Fig. 27 shows the PL metric data separated by angle, and it is here where the differences across participants can be assessed. The results are tightly grouped in most cases, with one outlier visible in the off-track standard atmosphere cases. The off-track values for Profile 1 are a little more spread out, but with the exception of outliers, the majority of the data shows good agreement across participants. 


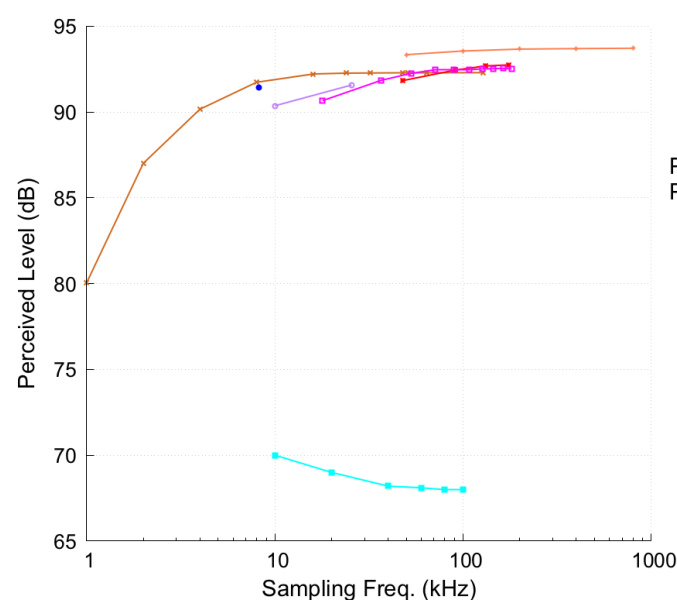

(a) Profile1 (Required).

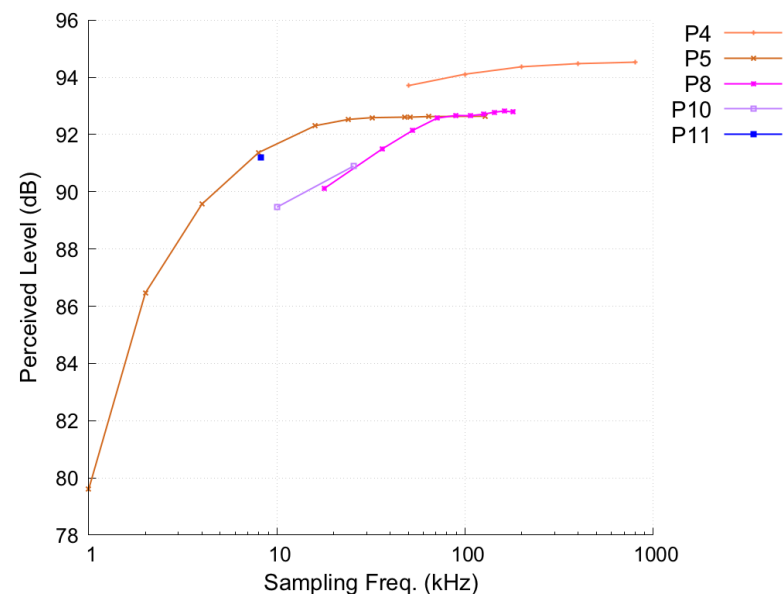

(c) Standard Profile (Optional).

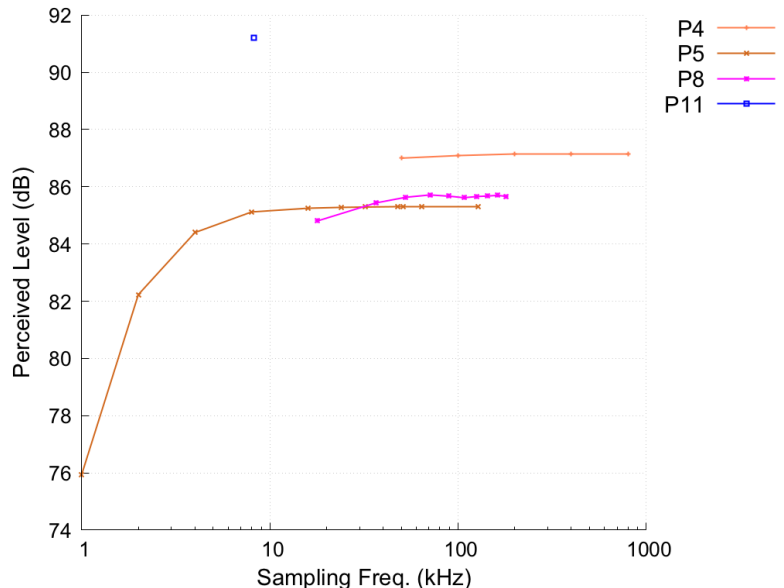

(b) Profile2 (Optional).

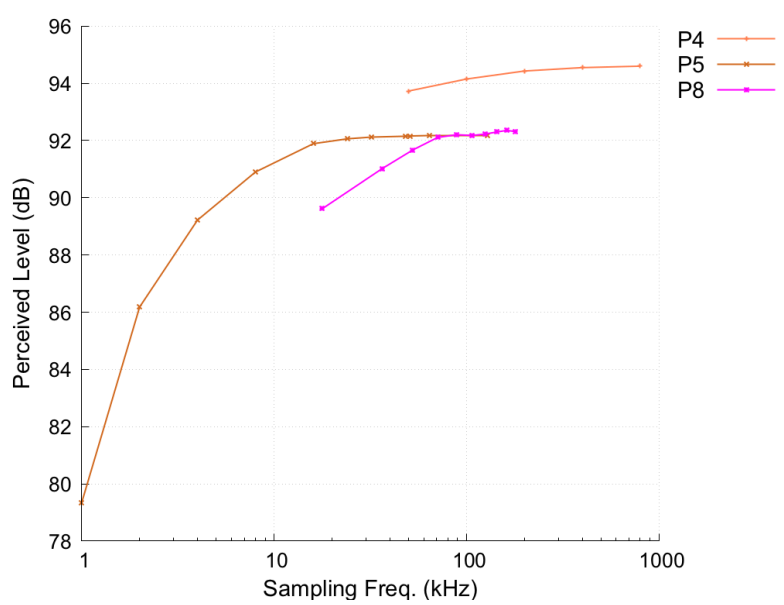

(d) Standard Profile w/ RH=70\% (Optional).

Figure 13. Loudness convergence for LM1021 at Roll Angle $=0^{\circ}$

\section{Axi-symmetric body analysis}

\section{V.A. Ground signatures and loudness metrics}

Figure 28 shows the ground signatures of all submissions from the participants for all atmospheric profiles directly beneath the flight track. Similar to Fig. 7, submission from participant P4 seems to be the outlier for standard profiles. For the measured profiles, the shock locations and signature durations have a larger variation compared to results from standard profiles.

For the off-track cases in figs. 29 and 30, discounting the P4 submission, the discrepancies between submissions increases compared to under-track location. For the case of Profile4, all participants predicted a cut-off before the boom reaches the ground at an azimuthal angle of $-45^{\circ}$, hence the blank figure corresponding to that profile in Fig. 29. The off-track angles are close to lateral cut-off and large variations are observed in the submissions, more so in the case of measured profiles with winds. The largest variation for azimuthal angle of $45^{\circ}$ is for Profile4, which is supposed to generate the lowest loudness in terms of PL. The signature duration and rise time variations also increase from standard profiles to measured profiles. These trends are similar to submissions for LM1021.

The submitted and calculated carpet loudness plots are depicted in figs. 31 and 32 respectively. As in the previous case, $\mathrm{P} 4$ and $\mathrm{P} 9$ are the outliers on the submission data, while $\mathrm{P} 4$ is the lone outlier when loudness values are computed consistently using a single loudness metric calculation scheme. Participant P7 submissions are higher, in terms of PL, than the other submissions, except P4. However, once loudness metrics are calculated uniformly, this discrepancy is eliminated. For the standard profile, the spread at 


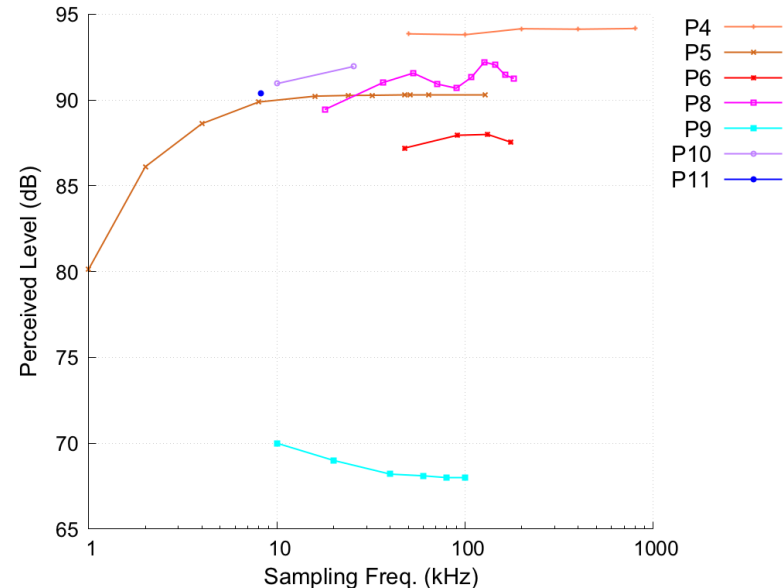

(a) Profile1 (Required).

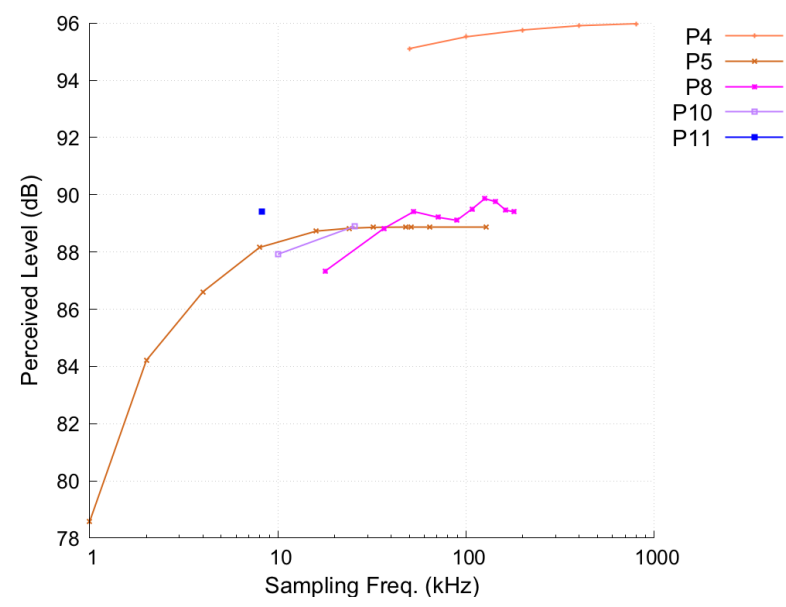

(c) Standard Profile (Optional).

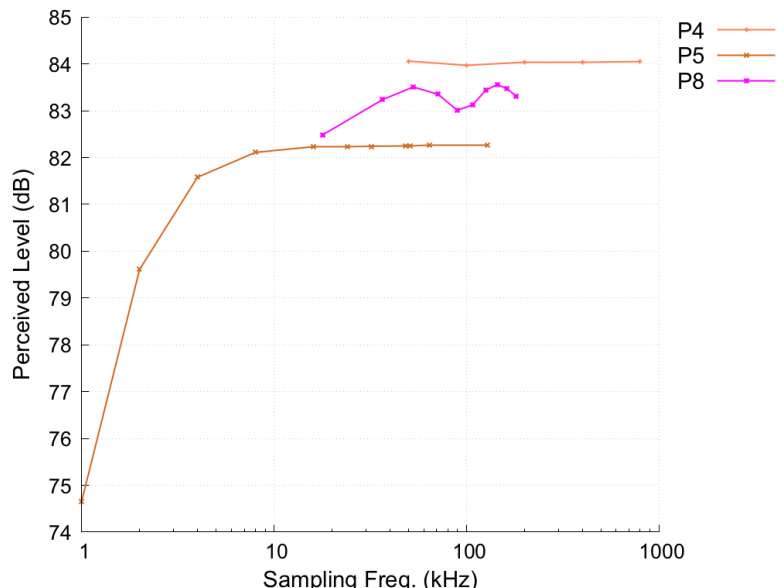

(b) Profile2 (Optional).

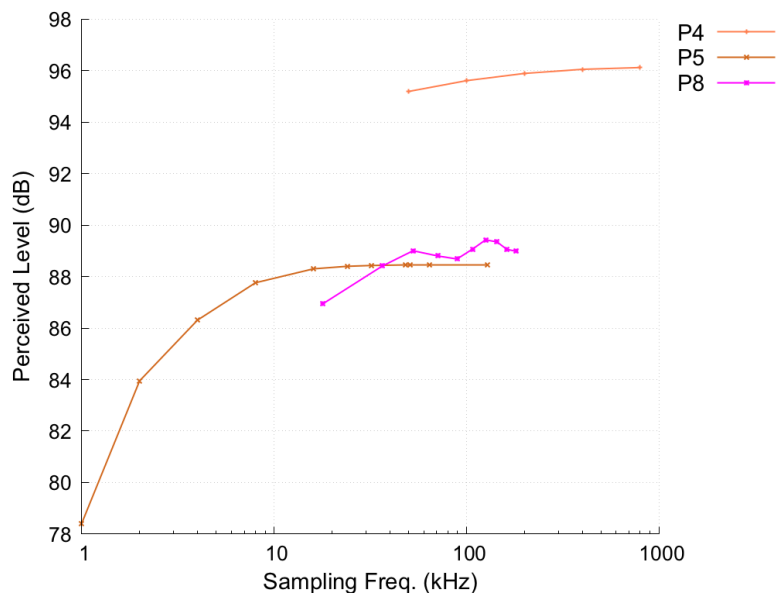

(d) Standard Profile w/ RH=70\% (Optional).

Figure 14. Loudness convergence for LM1021 at Roll Angle $=-30^{\circ}$

off-track azimuthal angles is higher for submitted values than for uniformly computed values, suggesting that there are inconsistencies in loudness calculation procedure between participants. The calculated loudness values display a higher spread at $-45^{\circ}$ (except for profile4) and $45^{\circ}$ compared to the submissions, especially for measured Profile3. This is interesting and unexpected as it was thought the variation would reduce by eliminating a source of uncertainty. It is the authors' belief that this result is fortuitous or the result of erroneous data. For the case of profile4, all submissions predict a cut-off at the requested off-track angle of $-45^{\circ}$. In fact, the lateral cut-off angle reported by different submissions is around $-44^{\circ}$, as can be verified from Fig. 31.

The loudness convergence plots for different azimuthal angles are shown in figs. 33 to 35. Unlike LM1021, loudness convergence is achieved at lower sampling

\section{V.B. Spectral Analysis}

Figs. 36 and 37 depict the loudness spectra and Sound Pressure Level (SPL) spectra respectively corresponding to under-track boom signatures. As before, the loudness spectra indicate the $1 / 3^{r d}$-octave frequency bands which are most important to the calculation of PL, which approximates the sensitivity of human hearing, while SPL spectra indicate the sound energy in different $1 / 3^{r d}$-octave frequency bands. Compared to LM1021 spectral analysis (See Fig. 18), the contributions in terms of sones are much smaller in this case, as this is a low-boom concept. From Fig. 36(a), P6 is a clear outlier, and as expected the computed PL is smaller than other participants' submissions (See Fig. 32(a)). As we move to optional cases, P4 becomes the outlier 


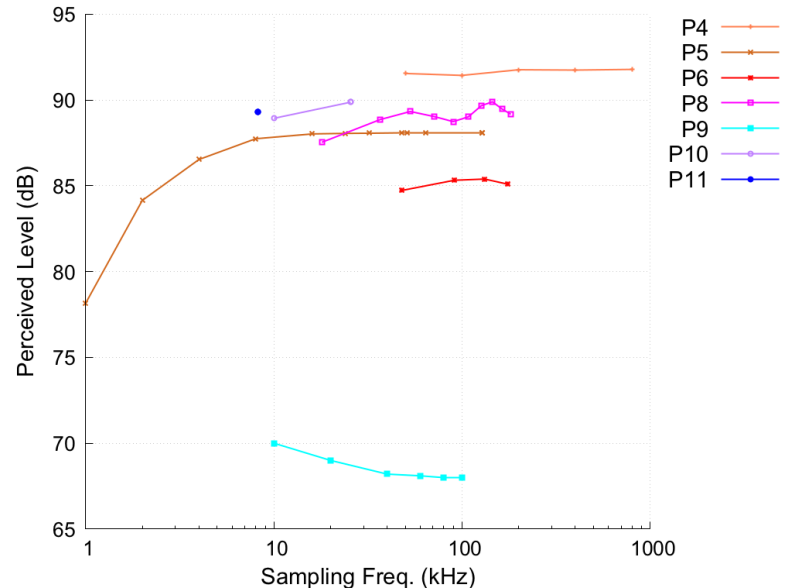

(a) Profile1 (Required).

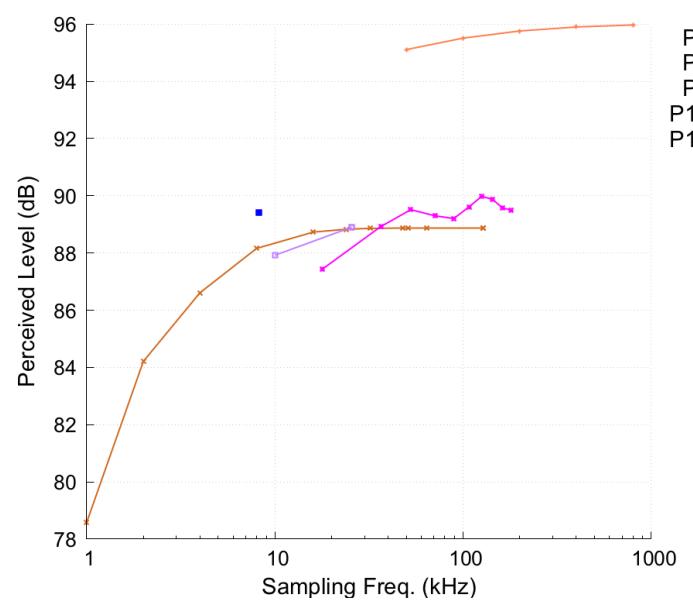

(c) Standard Profile (Optional).

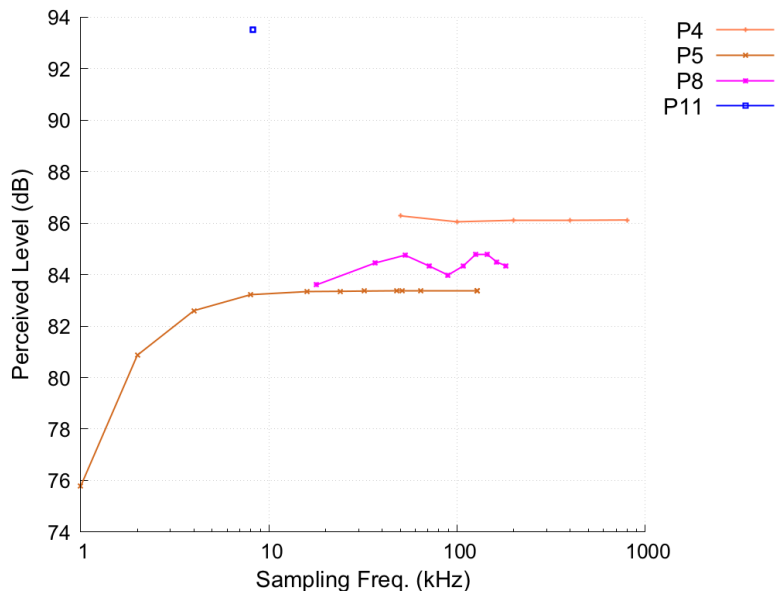

(b) Profile2 (Optional).

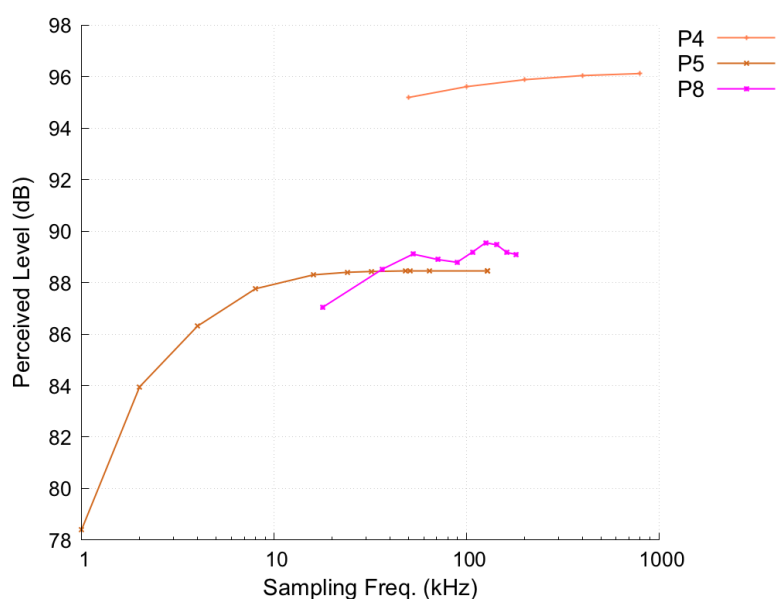

(d) Standard Profile w/ RH=70\% (Optional).

Figure 15. Loudness convergence for LM1021 at Roll Angle $=30^{\circ}$

with predicted higher contributions at most frequencies. For the standard profile, P10 seems to have lesser contributions at higher frequencies, suggesting that perhaps the shocks are dissipated or smoother compared to other submissions. Similar trends are also seen in the SPL plots.

As the analysis moves to off-track locations, the spread between submissions widens as seen in Figs. 38 and 39. The differences are more pronounced for measured profiles showing the impact of differences in the modeling of atmospheric winds. Unlike the LM1021 case, the differences are at higher frequencies, which suggests that the submissions are different at resolving shocks and other high frequency content. The results for positive roll angles (See figs. 40 and 41) are similar to these plots, except for Profile4, where the contributions from different frequencies are much smaller compared to other profiles.

\section{V.C. Statistical Analysis}

Fig. 42 shows the mean and one standard deviation of the spread for all the four atmospheric profiles at the under-track location. As for the LM1021 case, the lossless propagation case (P11) is omitted as inclusion of that would amplify the differences. Unlike the LM1021 case, the standard deviation is larger at the front shock system for the standard profiles, and rear shock system for measured Profile3. In this case, the humidity being constant at $70 \%$ or varying according to ANSI guidance ${ }^{10}$ does not seem to make a difference. Interestingly, the measured Profile3, reduces the standard deviation in the front shock system, while increasing in the aft shock system. As before, winds have a certain role in the discrepancies between the submissions. 


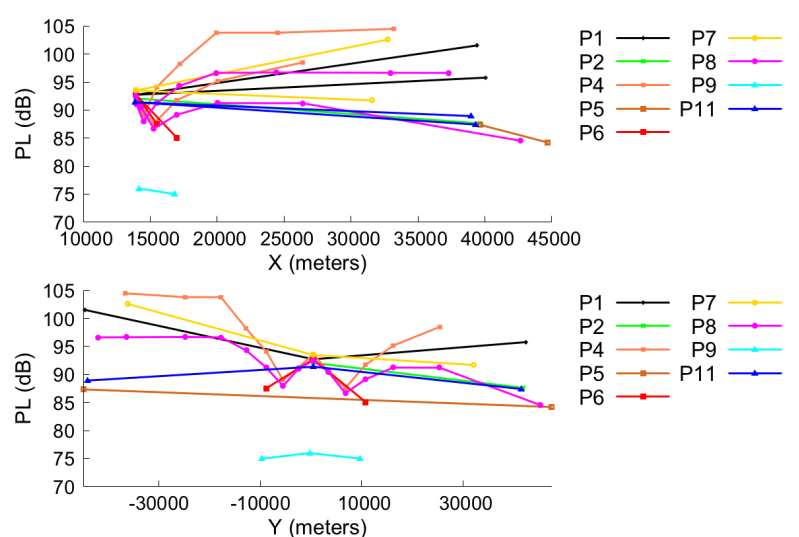

(a) Profile1 (Required).
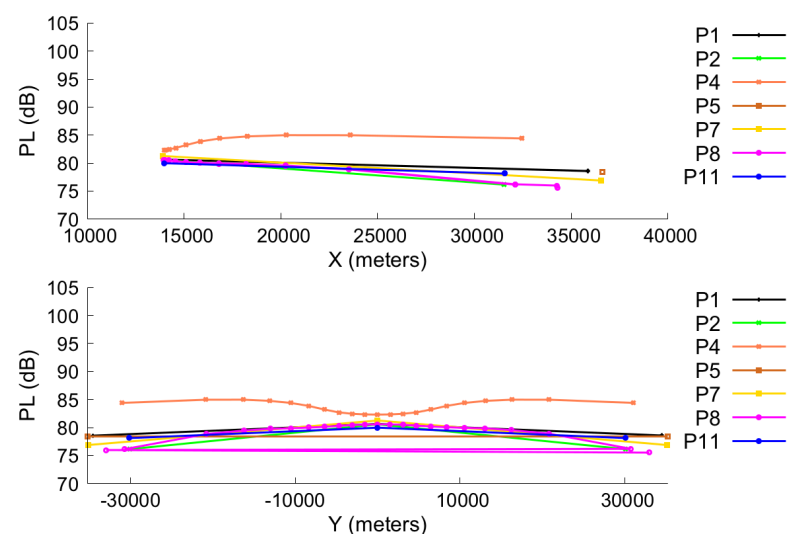

(c) Standard Profile (Optional).
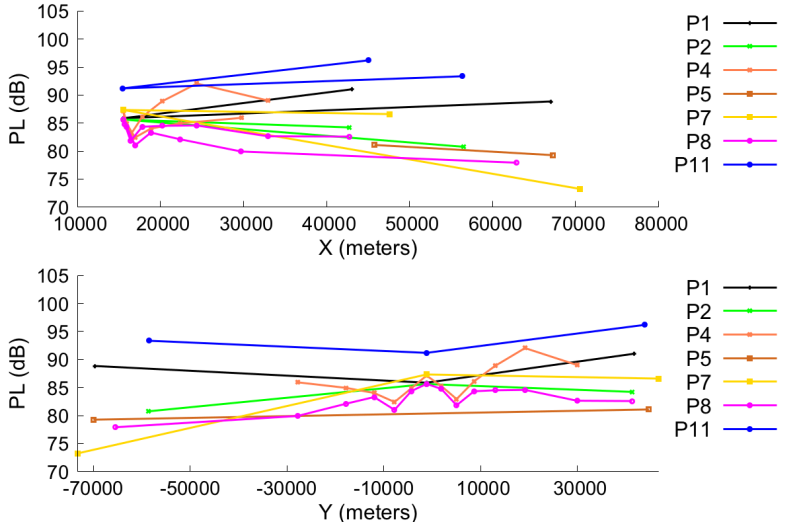

(b) Profile2 (Optional).
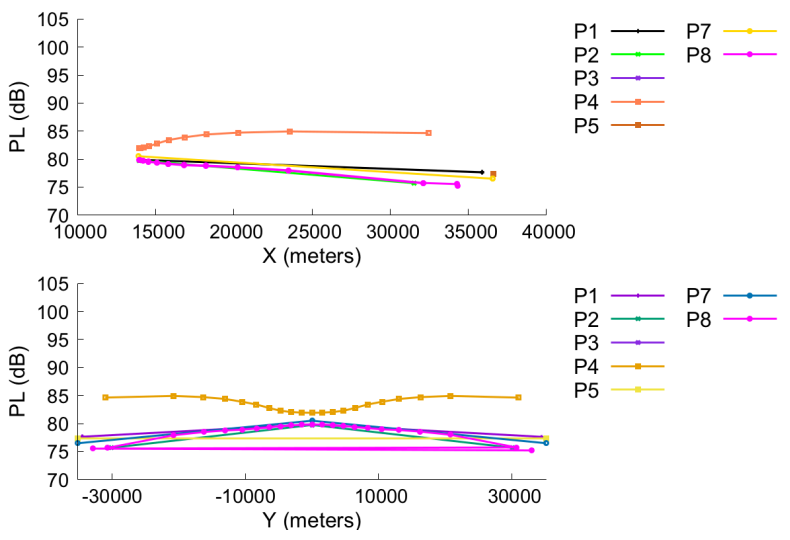

(d) Standard Profile w/ RH=70\% (Optional).

Figure 16. Submitted Loudness-Ranges for LM1021

As we move off-track (See figs. 43 and 44), the standard deviation bounds increase compared to the under-track locations. As expected from the under-track results, the standard deviations from standard profiles are slightly higher compared to the measured profiles.

As with the LM1021 data, the AXIBODY data are further examined through the use of violin plots. Violin plots for six noise metrics are shown in Fig. 45; data from all submissions for all four atmospheric profiles at the angles of $-45,0$, and 45 degrees are included. The size of the distribution varies with each metric, but all have a larger spread with AXIBODY than they do with LM1021. Long tails, with possible multiple modes, are observed in the distributions, especially for PL, ASEL, and ISBAP. The extent and shape of these distributions indicates that the atmospheric profiles have a very large effect on most of the metrics. The distribution is once again smallest for the DSEL metric, which may indicate that this metric is less sensitive to changes in the waveform due to atmospheric profile differences.

The data are then subdivided by atmospheric profile as shown in Fig. 46. The distributions encompass smaller ranges of less than $10 \mathrm{~dB}$, except for Profile 4 . Profile 4 results in the lowest median values for all metrics, but the spread is 10-20 dB; PL, ASEL, and ISBAP have the larges range, with DSEL showing the smallest spread. Fig. 47 shows the PL metric data separated by angle, which helps explain the spread in values for Profile 4. For Profile 4, no results were reported for -45 degrees because the cutoff was predicted at a smaller angle in all cases. The PL at 45 degrees is much lower than undertrack, which is not the case for the other atmospheric profiles. The results are tightly grouped in most other cases, with one outlier visible in the off-track calculations. One participant predicted there would not be a boom for Profile 3 at 45 degrees, so the value is set to zero. When the data are separated in this fashion, the majority of the data shows relatively good agreement across participants.

Lastly, predictions using a standard atmosphere with a humidity profile according to ANSI S1.26 are compared to predictions using a standard atmosphere with a constant $70 \%$ relative humidity. Fig. 48 shows 


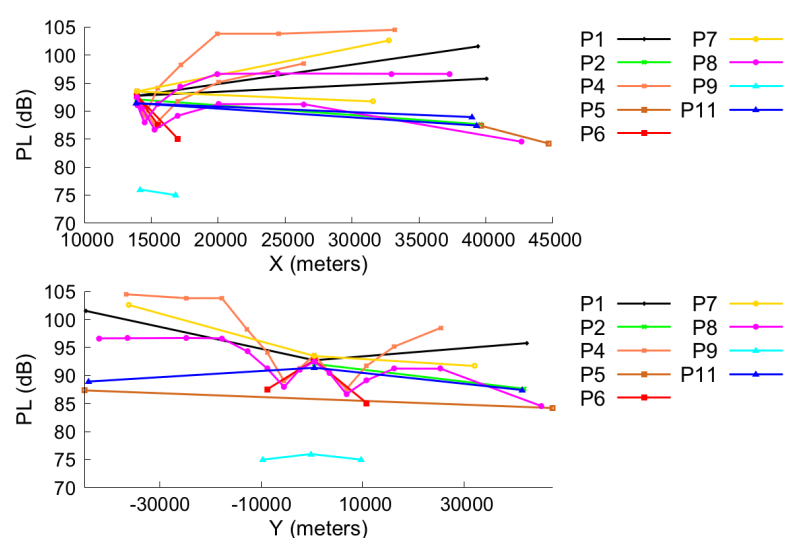

(a) Profile1: Submitted loudness.

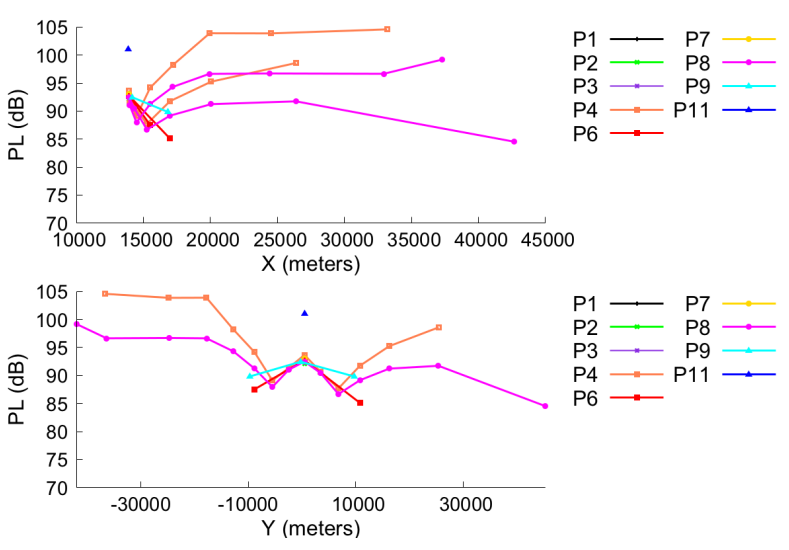

(b) Profile1: Calculated loudness.

Figure 17. Loudness-Ranges for LM1021

statistics for the difference for the six metrics, including both aircraft cases at three angles each. The median difference ranges from $0.2 \mathrm{~dB}$ for DSEL to 0.6 dB for ASEL. DSEL gives the smallest spread, while PL and ASEL have large spreads, reaching above $1 \mathrm{~dB}$ difference in ASEL. These results show that the choice of humidity profile can have a measurable effect on the ground signature loudness, and this effect varies with choice of noise metric. DSEL is the least sensitive to atmospheric condition changes, while PL and ASEL are the most sensitive.

\section{Lessons Learned and Recommendations}

Based on the submissions and subsequent analysis pulling the data together, several lessons were learned. The following is a list of those along with recommendations for future exercises on this subject.

1. Different propagation algorithms implement winds in different ways. Without a standard wind convention, most participants ran their propagation analysis with a lateral wind direction that was the reverse of what was intended. To reduce workload in re-calculating with the correct wind direction after the workshop, the positive and negative azimuthal angles were flipped from the results of the few folks who originally implemented the winds as intended. Future workshops should standardize the definition of winds so that such a discrepancy does not arise.

2. The initial atmospheric pressure profile specification for standard profiles was extremely coarse. Since some implementations allowed temperature and pressure profiles to be input independent of each other and others linearly interpolated the atmospheric profiles, including pressure, this resulted in large errors during the conversion from the input near-field in non-dimensional terms into a dimensional waveform for propagation. During the initial analysis this discrepancy was caught, and a much refined pressure profile, based on the hydrostatic equation, was distributed to the participants. In future workshops, it may be better to supply atmospheric profiles with sufficient granularity to avoid such inconsistencies.

3. During the data analysis phase, it was realized that some participants submitted ranges only corresponding to lateral cut-off azimuthal angles and not for the required angles, while supplying ground signatures corresponding only to the requested angles, and not at lateral cut-off roll angles. In order to plot consistent and uniformly computed loudness metrics versus range sketches, future workshop submissions should require participants to submit ranges and loudness metrics for all azimuthal angles including lateral cut-offs.

4. For loudness convergence, participants submitted their calculated loudness values at different sampling frequencies. However, it was realized that some participants' loudness calculations may be different compared to others. Since the participants were not required to submit ground signatures corresponding to intermediate sampling frequencies, the convergence plots could not be updated with a uniform loudness computation scheme. Future workshops should request ground signatures at intermediate sampling frequencies from the participants. 


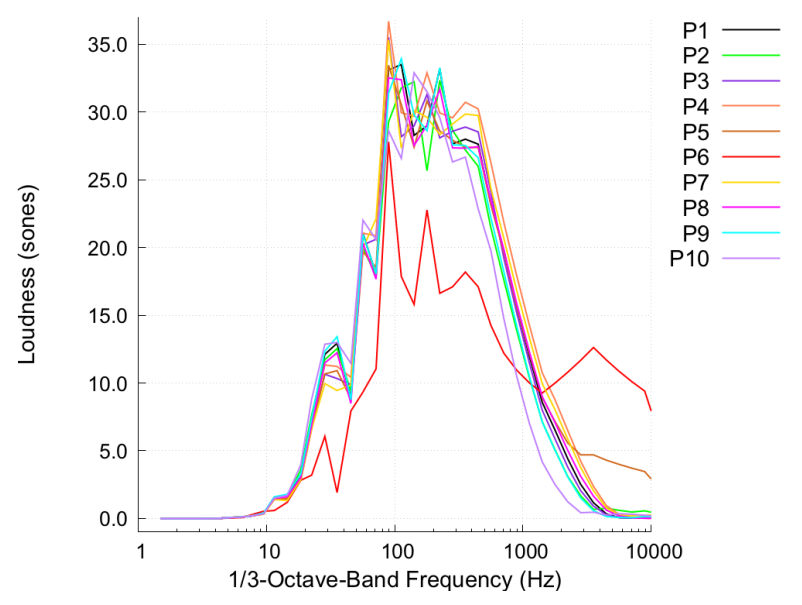

(a) Profile1 (Required).

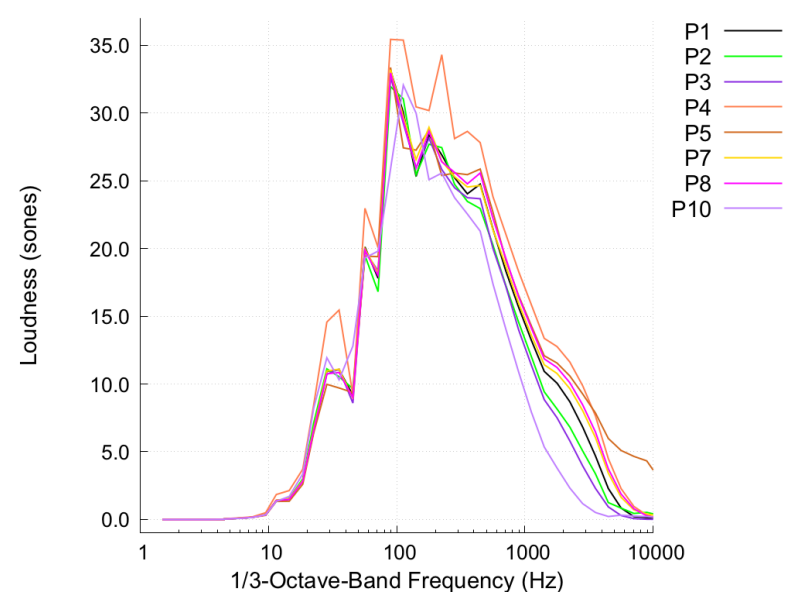

(c) Standard Profile (Optional).

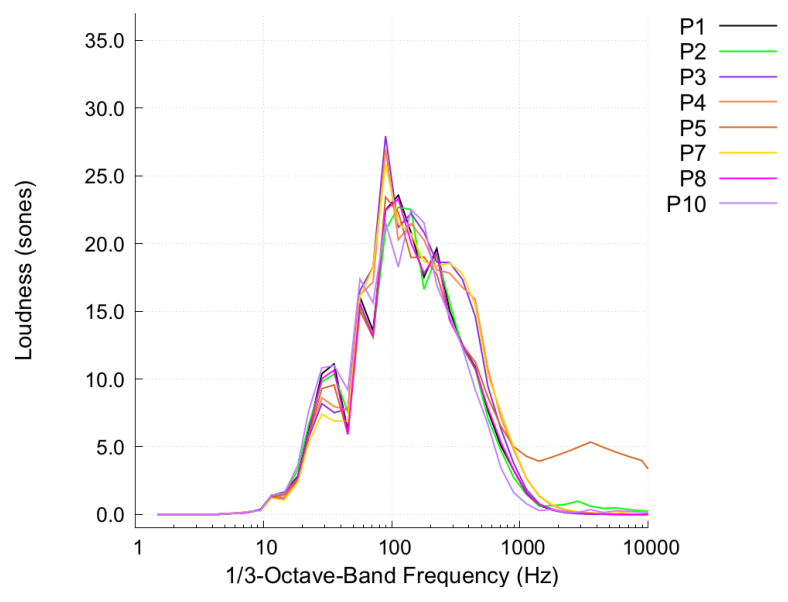

(b) Profile2 (Optional).

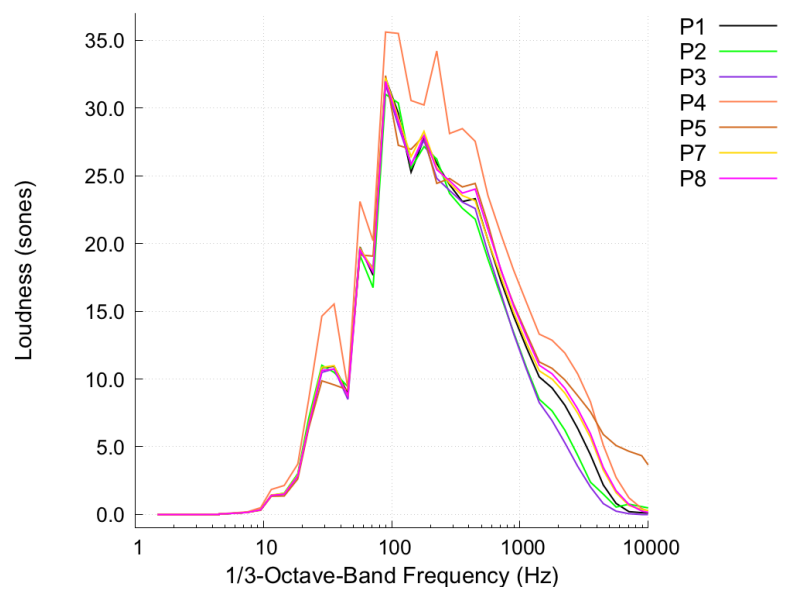

(d) Standard Profile w/ RH=70\% (Optional).

Figure 18. Frequency spectra of Ground Signatures for LM1021 at Roll Angle $=0^{\circ}$

\section{Next Steps}

Given the differences that were observed between participants' submissions, particularly at off-track azimuthal angles, the first order of business is to break into a smaller core group (two or three people maximum) to verify that distinct and independent lossy implementations produce results that are within a small percentage of each other in terms of ray paths, ground signatures and loudness metrics at multiple azimuthal angles including near lateral cut-offs. Without sufficient agreement within this smaller group, there is no reason to conduct and explore comparisons with multiple different implementations as the sources of disagreement are too many even without adding multiple numerical models into the mix. Once it can be ascertained that the results are in close proximity to each other, it makes sense to open up the analysis to a wider list of participants as part of the next workshop. At that time, additional complexities such as advanced terrain, focusing physics with maneuvering trajectories, spherical earth 3D propagation models, booms in the shadow region etc. may be compared. From the authors' perspective, the cases considered in this workshop represent sufficient complexity.

In addition, it could be helpful to present baseline loudness calculation cases that would be available to all participants before the next workshop. Using these cases, participants would be able to check their loudness results and make adjustments prior to the workshop. This would help align loudness calculation results to eliminate one source of variability in workshop results. 


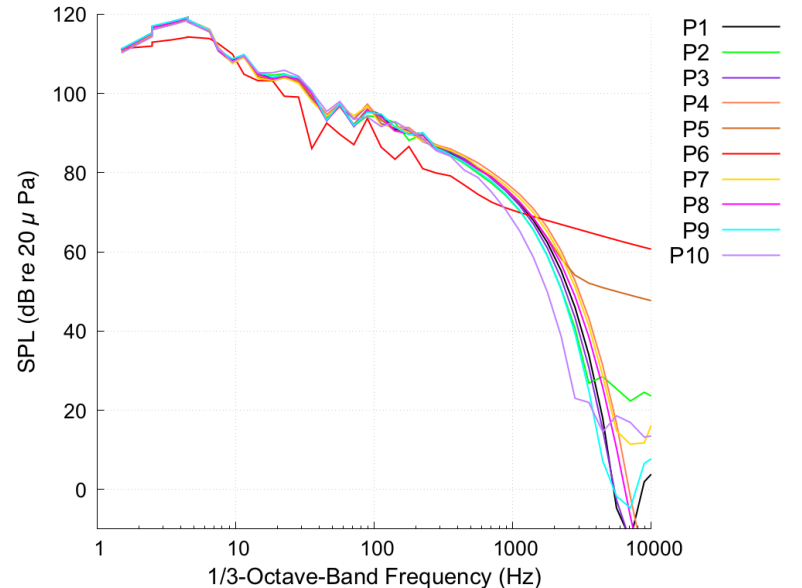

(a) Profile1 (Required).

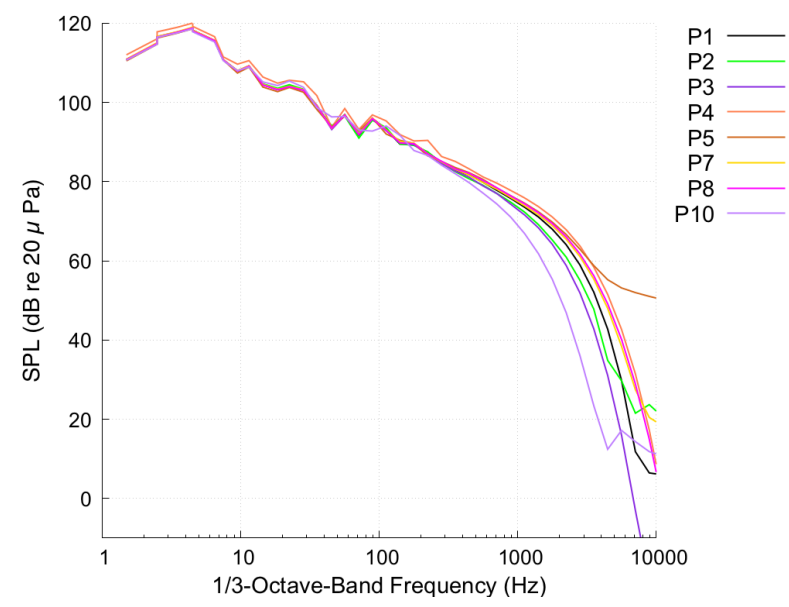

(c) Standard Profile (Optional).

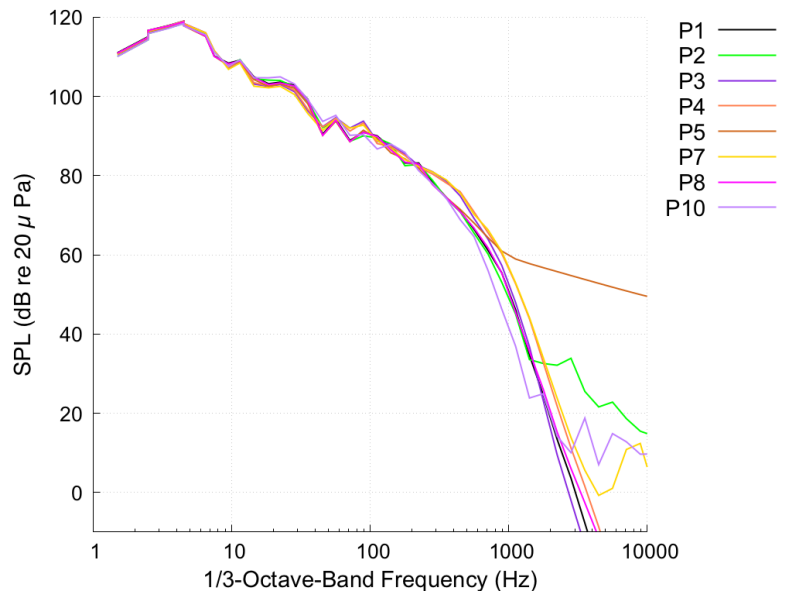

(b) Profile2 (Optional).

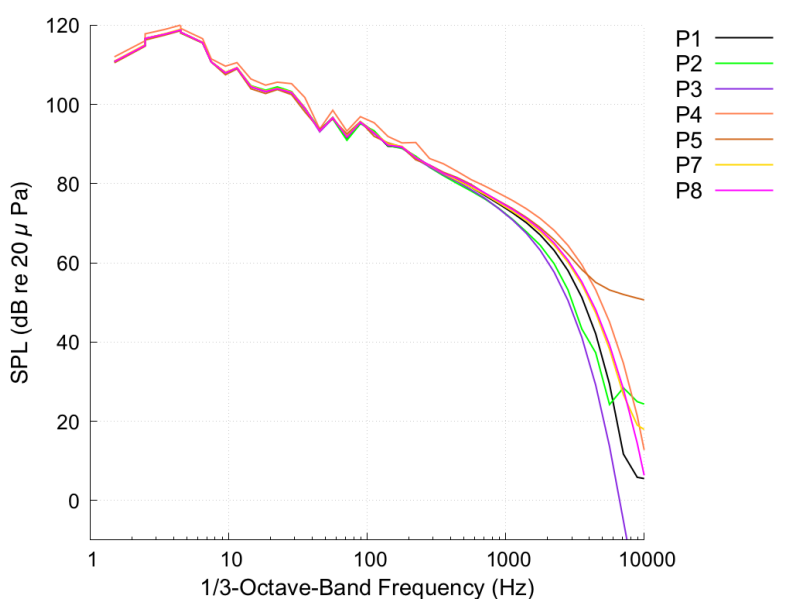

(d) Standard Profile w/ RH=70\% (Optional).

Figure 19. Frequency spectra of Ground Signatures for LM1021 at Roll Angle $=0^{\circ}$

\section{Conclusions}

The propagation portion of the Second AIAA Sonic Boom Prediction Workshop was a success in terms of evaluating and comparing results from multiple sonic boom propagation models for multiple aircraft concepts under measured and standard atmospheric conditions. The differences between results are documented and the associated statistics are presented. Since participants were free to use their best practices and methods, delving further to extract and eliminate the source of differences was unfeasible and beyond the scope of this effort. However, a new effort with a reduced scope and renewed focus is needed to track down the differences between different implementations.

Regardless of differences between participant submissions, all results confirm that the ground sonic boom waveforms, locations, and loudness values depend greatly on the atmospheric profile used in the prediction. Results with measured profiles differ significantly from those with a standard atmosphere profile, particularly at off-track locations. The effect of changing the standard relative humidity profile to be a fixed $70 \%$ was small, but measurable. The extent of variability between results differed according to which noise metric was analyzed, with PL resulting in one of the larger variabilities and DSEL resulting in a tighter grouping of results. This observation, however, does not confirm a level of correlation with human perception, which is studied elsewhere. This workshop and analysis provides information to industry, research organizations, and regulators about sonic boom predictions under realistic atmospheric conditions and highlights the areas where further work may be necessary to bring results into better agreement. Continuation of this effort is proposed to inform the development of a noise certification standard for civil supersonic aircraft. 


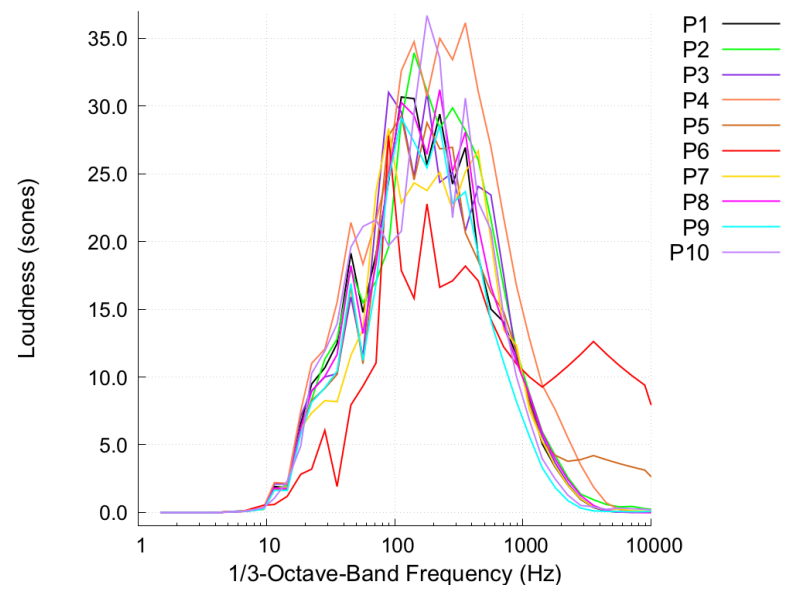

(a) Profile1 (Required).

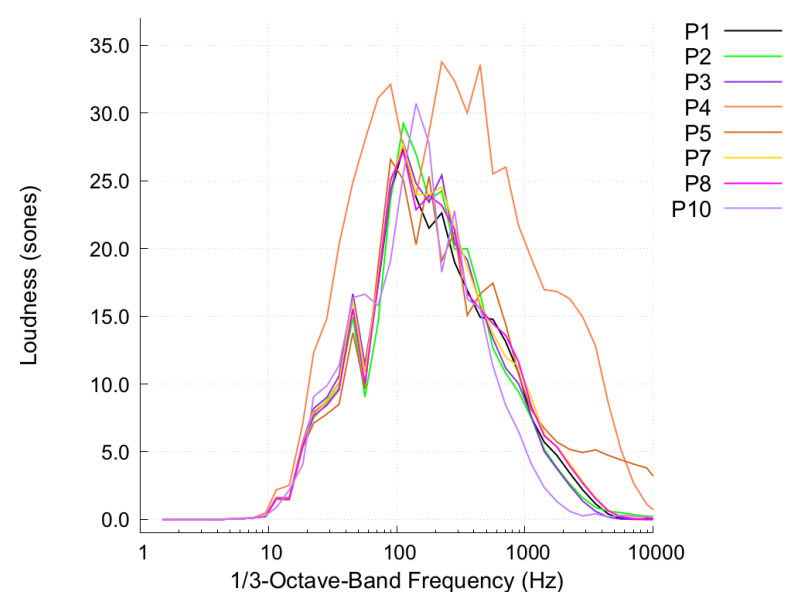

(c) Standard Profile (Optional).

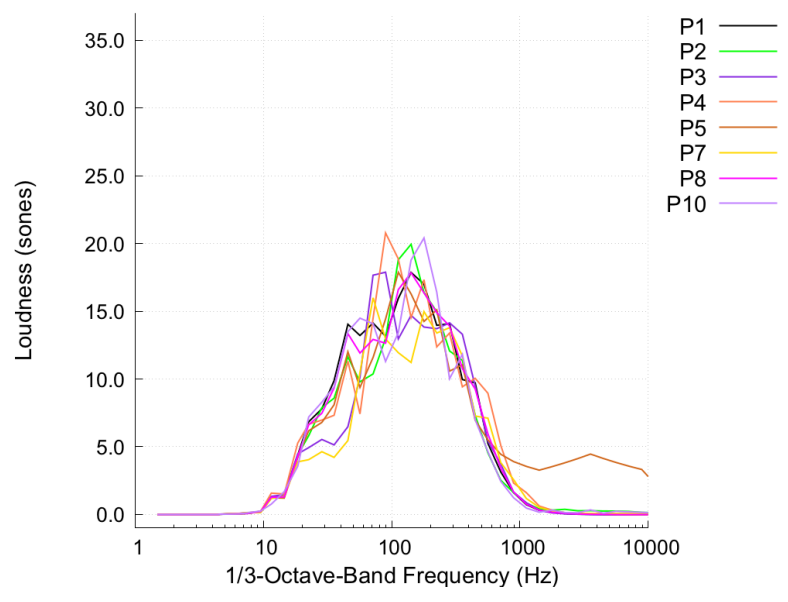

(b) Profile2 (Optional).

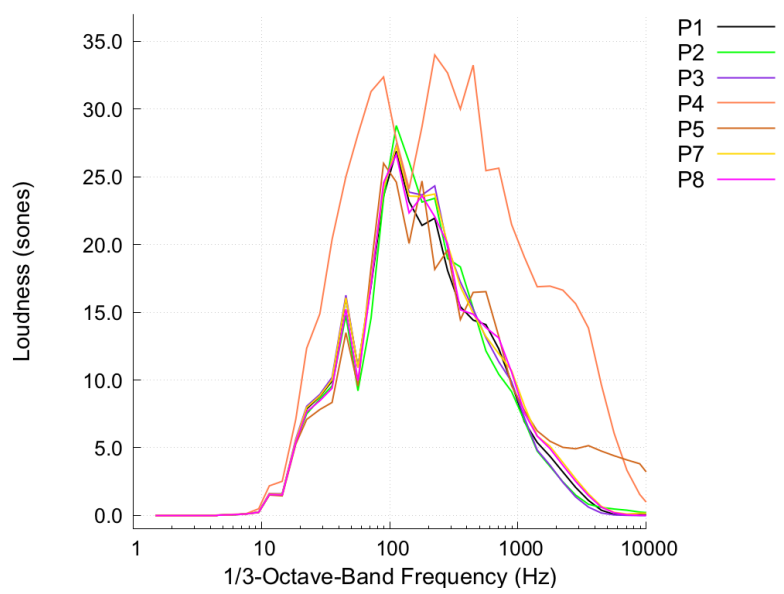

(d) Standard Profile w/ RH=70\% (Optional).

Figure 20. Frequency spectra of Ground Signatures for LM1021 at Roll Angle $=-30^{\circ}$

\section{Acknowledgments}

This work was supported by the NASA Advanced Air Vehicles Program, Commercial Supersonic Technology (CST) Project and by the NASA Project entitled "Sonic Boom Propagation Tools and Methods for Low Sonic Boom Design," under NASA contract number NNL08AA00B, task number NNL12AA55T.

\section{References}

${ }^{1}$ Waithe, K. A., "Introduction of First Low Boom Prediction Workshop," AIAA Paper 2013-650, 2013.

${ }^{2}$ Morgenstern, J., Norstrud, N., Sokhey, J., Martens, S., and Alonso, J. J., "Advanced Concept Studies for Supersonic Commercial Transports Entering Service in the 2018 to 2020 Period," NASA CR-2013-217820, Langley Research Center, Feb. 2013.

${ }^{3}$ Wintzer, M., Ordaz, I., and Fenbert, J. W., "Under-Track CFD-Based Shape Optimization for a Low-Boom Demonstrator Concept," AIAA Paper 2015-2260, 2015.

${ }^{4}$ Aftosmis, M. J., Berger, M. J., and Melton, J. E., "Robust and Effcient Cartesian Mesh Generation for Component-Based Geometry," AIAA Journal, Vol. 36, No. 6, 1998, pp. 952-960.

${ }^{5}$ Aftosmis, M. J. and Berger, M. J., "Multilevel Error Estimation and Adaptive h-Refinement for Cartesian Meshes with Embedded Boundaries," AIAA Paper 2002-863, 2002.

${ }^{6}$ Nemec, M., Aftosmis, M. J., Murman, S. M., and Pulliam, T. H., “Adjoint Formulation for an Embedded-Boundary Cartesian Method," AIAA Paper 2005-877, 2005.

${ }^{7}$ Durre, I., Vose, R. S., and Wuertz, D. B., "Overview of the Integrated Global Radiosonde Archive," Journal of Climate, Vol. 19, 2006, pp. 53-68. 


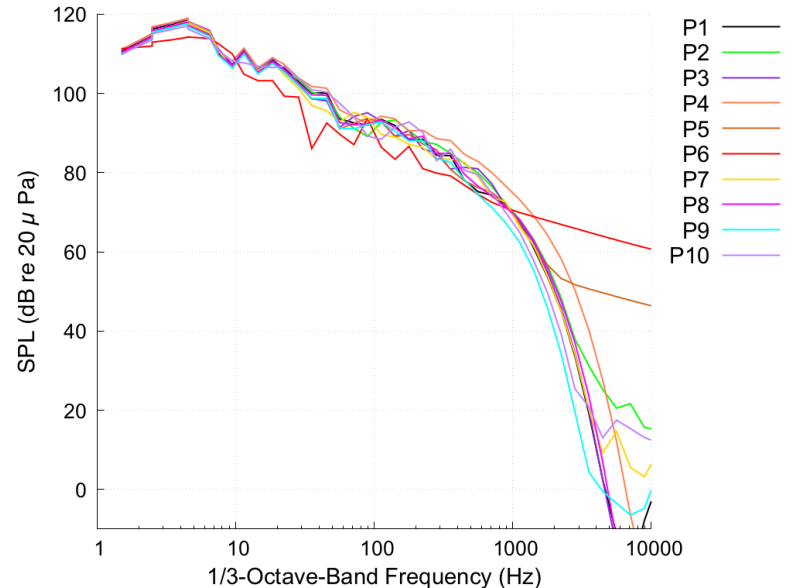

(a) Profile1 (Required).

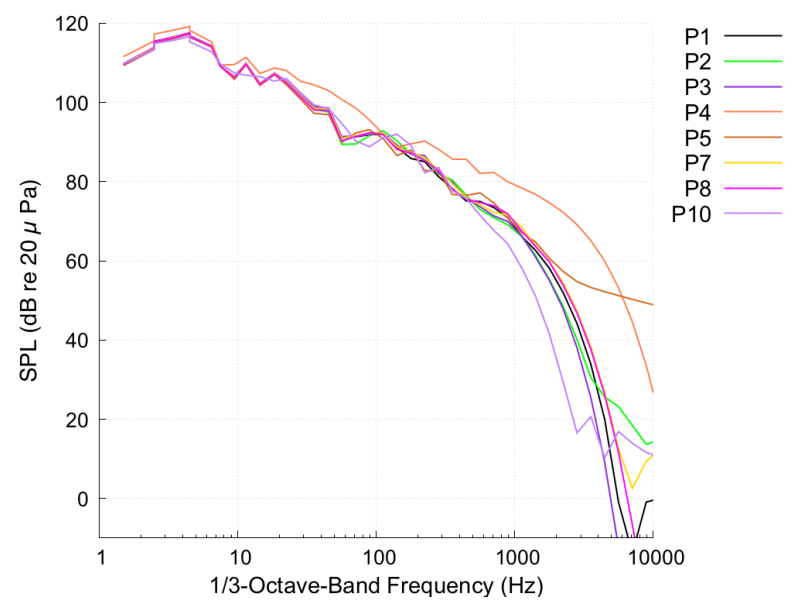

(c) Standard Profile (Optional).

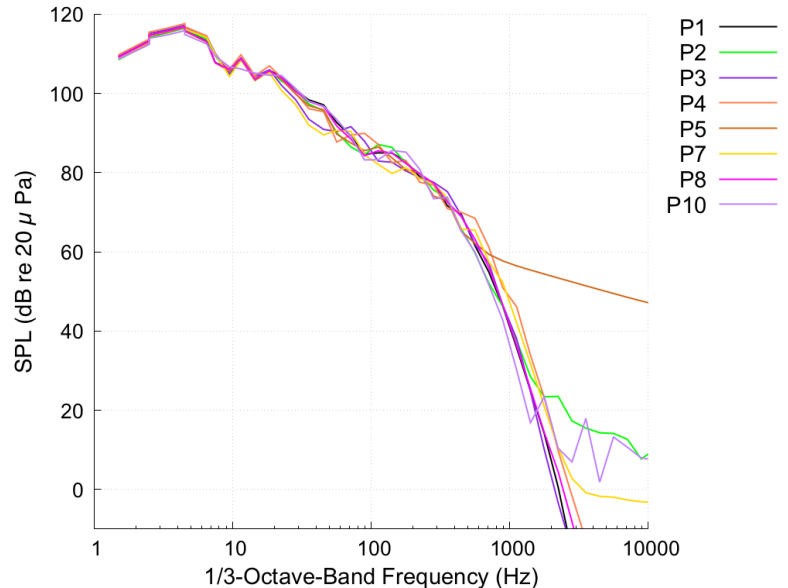

(b) Profile2 (Optional).

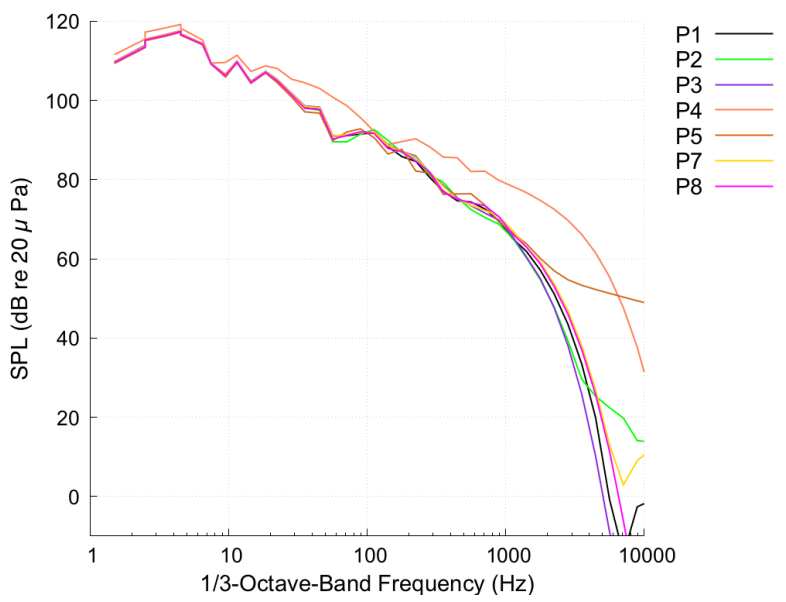

(d) Standard Profile w/ RH=70\% (Optional).

Figure 21. Frequency spectra of Ground Signatures for LM1021 at Roll Angle $=-30^{\circ}$

${ }^{8}$ Rallabhandi, S. K., "Advanced Sonic Boom Prediction Using Augmented Burger's Equation," Journal of Aircraft, Vol. 48, No. 4, 2011, pp. 1245-1253.

${ }^{9}$ U.S. Standard Atmosphere, 1976, U.S. Government Printing Office, 1976.

${ }^{10}$ Committee S1, Acoustics, "Method for Calculation of the Absorption of Sound by the Atmosphere, Annex C," ANSI Standard S1.26-1995, American National Standards Institute, New York, NY, September 1995.

${ }^{11}$ International Civil Aviation Organization, International Standards and Recommended Practices, Annex 16 to the Convention on International Civil Aviation, Environmental Protection, Volume I, Aircraft Noise, 2014, Seventh Edition.

${ }^{12}$ Leatherwood, J. D., Sullivan, B. M., Shepherd, K. P., McCurdy, D. A., and Brown, S. A., "Summary of Recent NASA Studies of Human Response to Sonic Booms," The Journal of the Acoustical Society of America, Vol. 111, No. 1, Jan. 2002, pp. 586-598.

${ }^{13}$ Stevens, S., "Perceived level of noise by Mark VII and decibels(E)," Journal of the acoustical society of America, Vol. 51, No. 2, 1972, pp. 575-601.

${ }^{14}$ Loubeau, A., Naka, Y., Cook, B. G., Sparrow, V. W., and Morgenstern, J. M., "A New Evaluation of Noise Metrics for Sonic Booms Using Existing Data," 2nd International Sonic Boom Forum, 20th International Symposium on Nonlinear Acoustics, July 2015.

${ }^{15}$ Shepherd, K. P. and Sullivan, B. M., "A Loudness Calculation Procedure Applied to Shaped Sonic Booms," NASA TP-3134, Langley Research Center, Nov. 1991. 


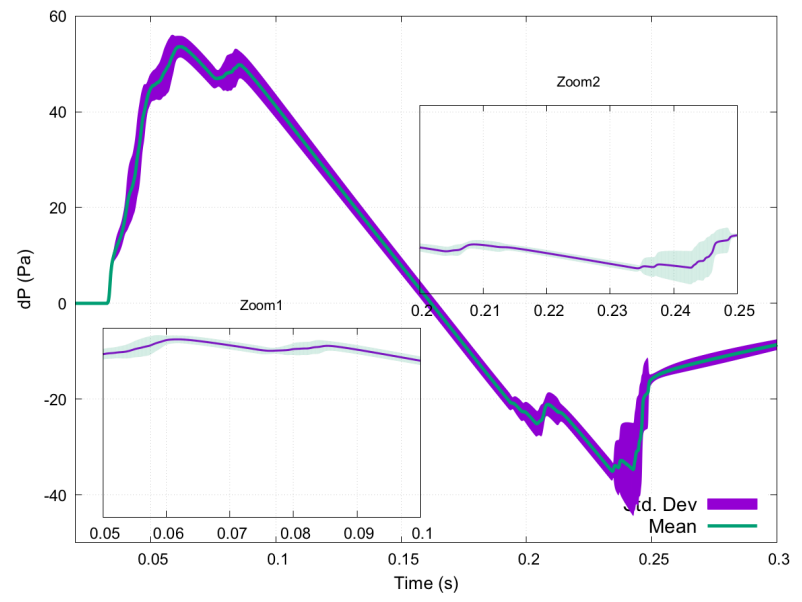

(a) Profile1 (Required).

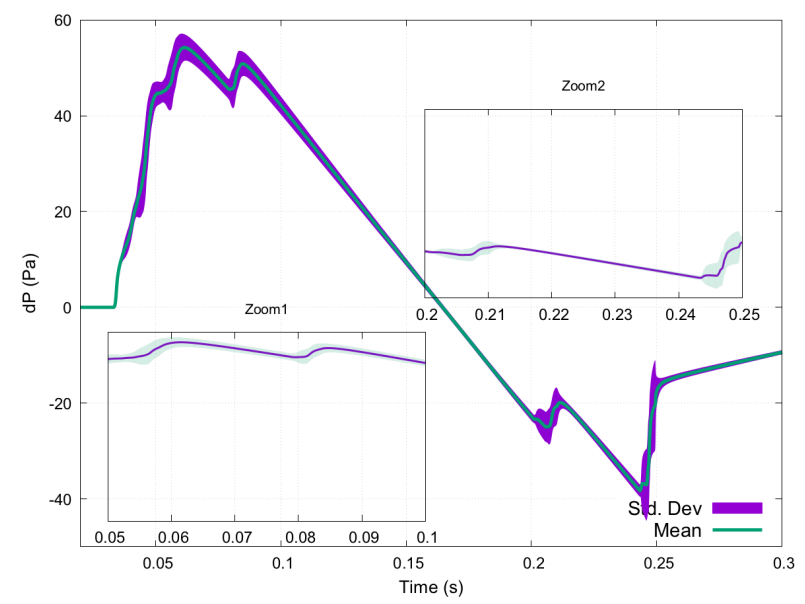

(c) Standard Profile (Optional).

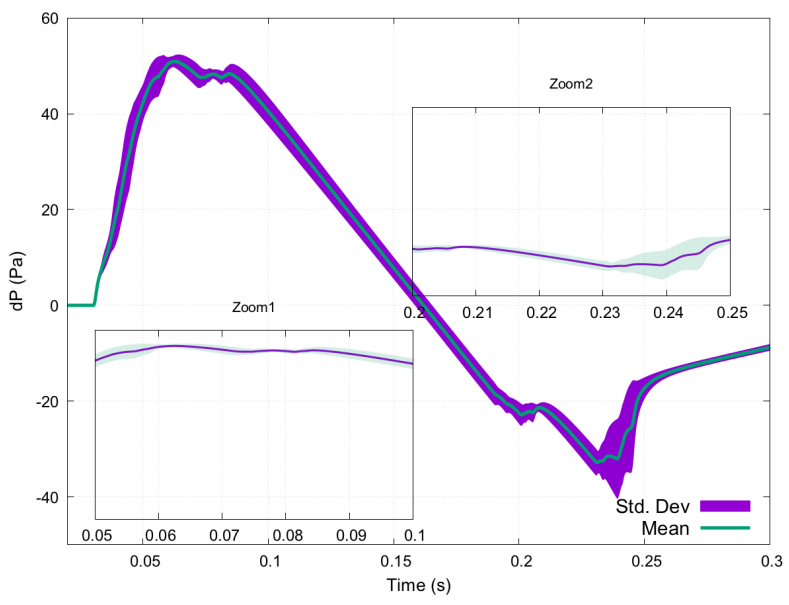

(b) Profile2 (Optional).

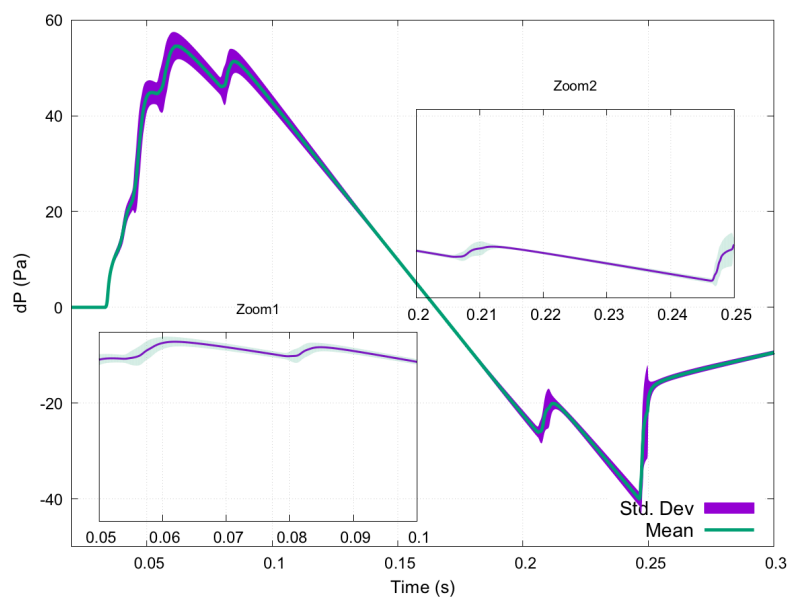

(d) Standard Profile w/ RH=70\% (Optional).

Figure 22. Mean and Standard deviation of Ground Signatures for LM1021 at Roll Angle $=0^{\circ}$ 


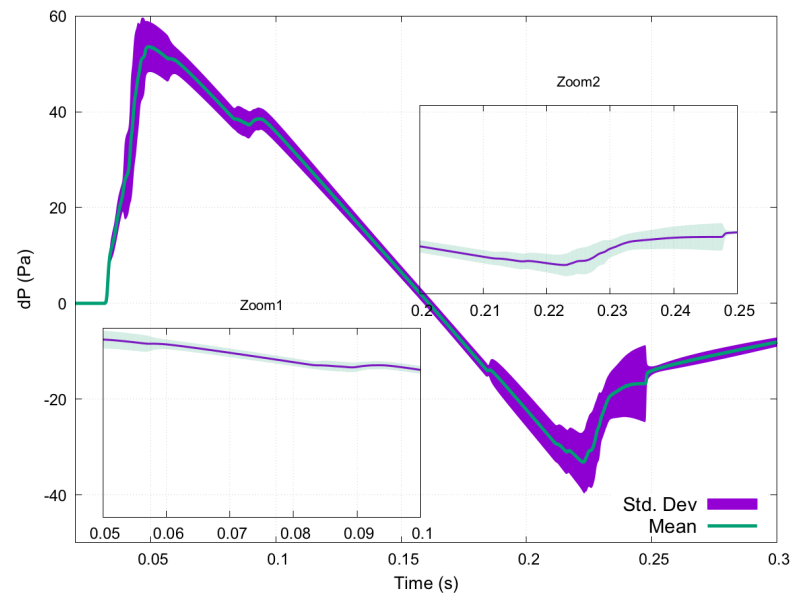

(a) Profile1 (Required).

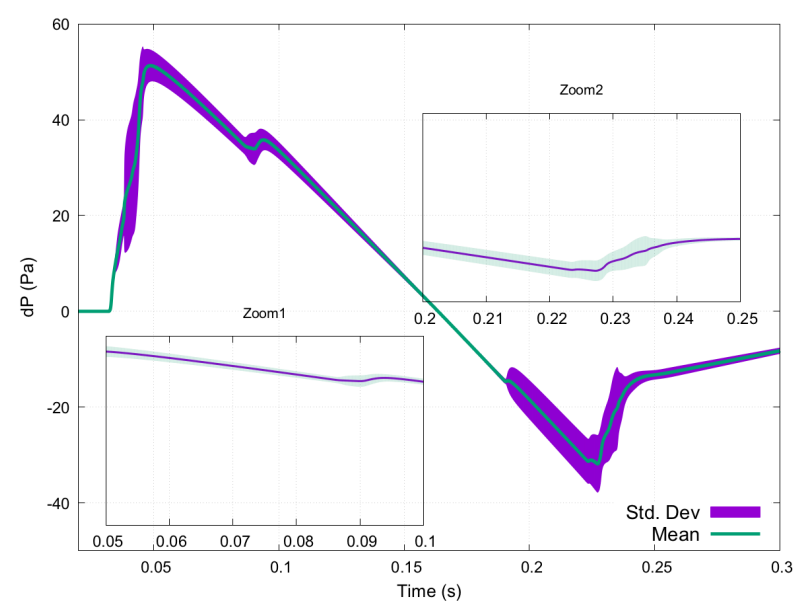

(c) Standard Profile (Optional).

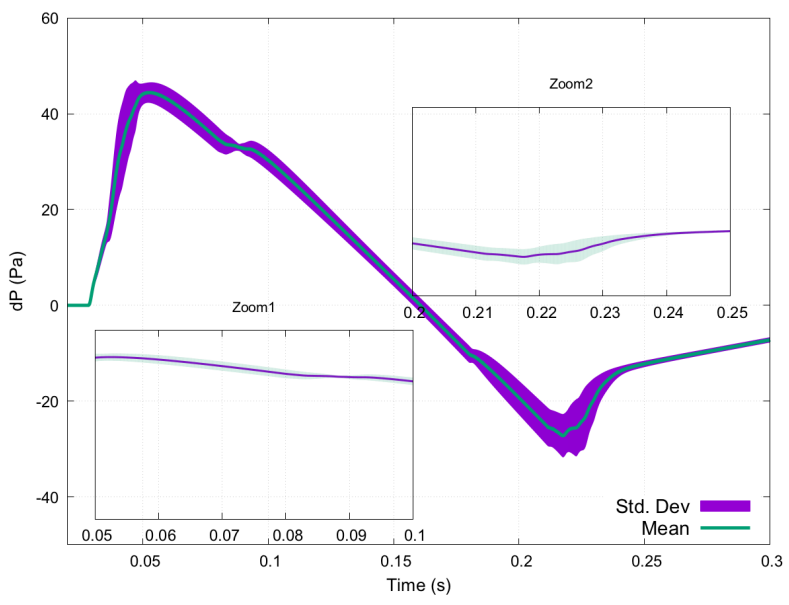

(b) Profile2 (Optional).

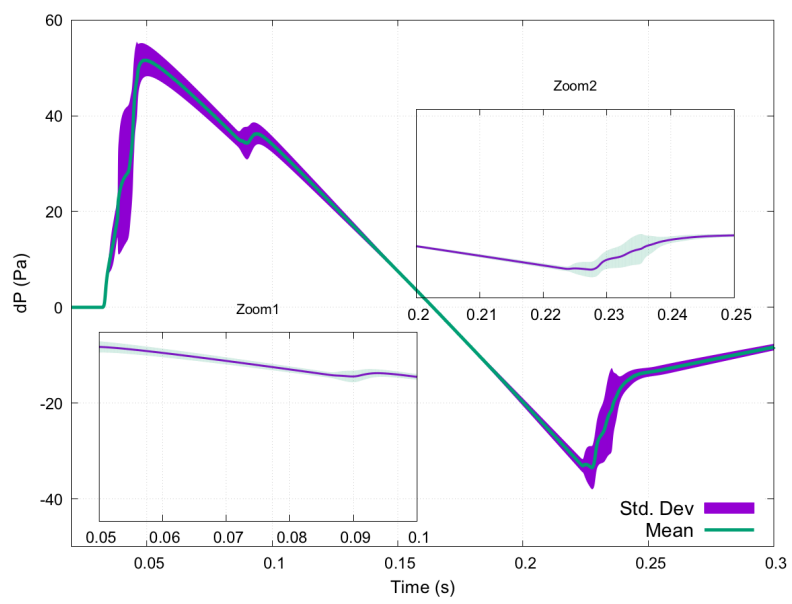

(d) Standard Profile w/ RH=70\% (Optional).

Figure 23. Mean and Standard deviation of Ground Signatures for LM1021 at Roll Angle $=-30^{\circ}$ 


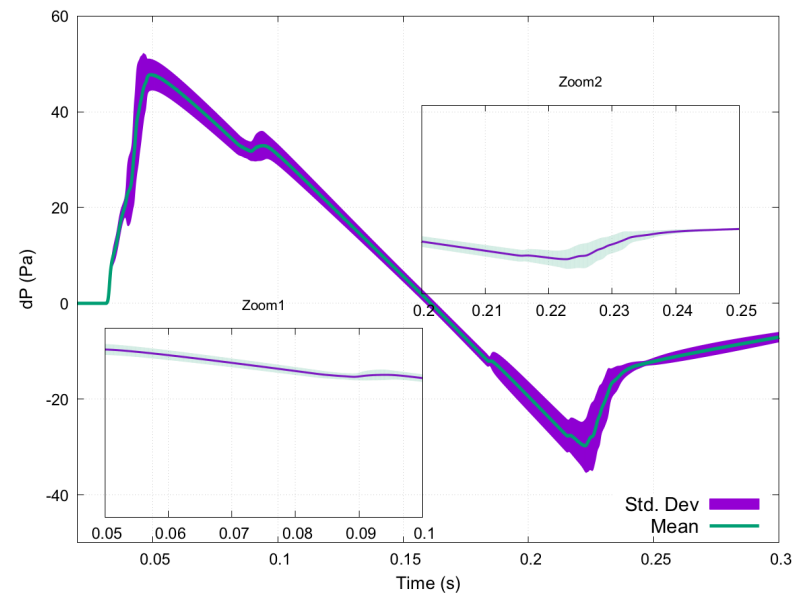

(a) Profile1 (Required).

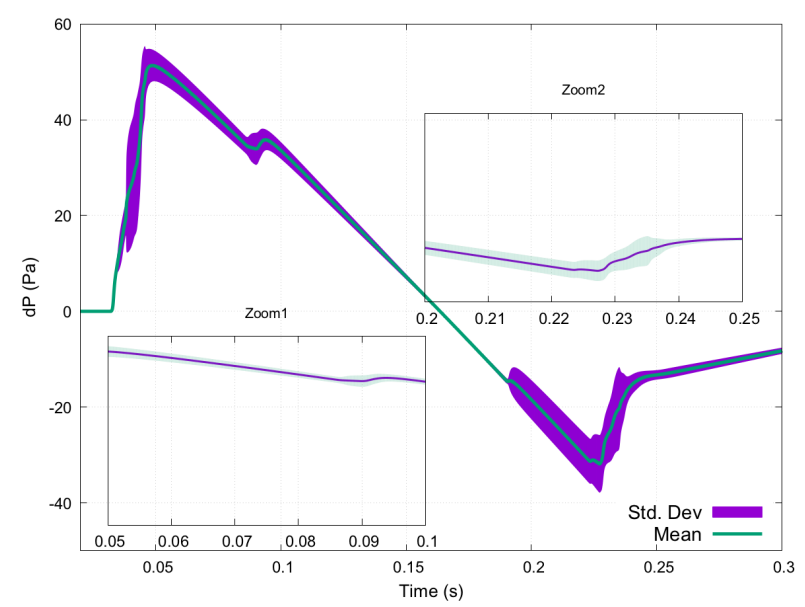

(c) Standard Profile (Optional).

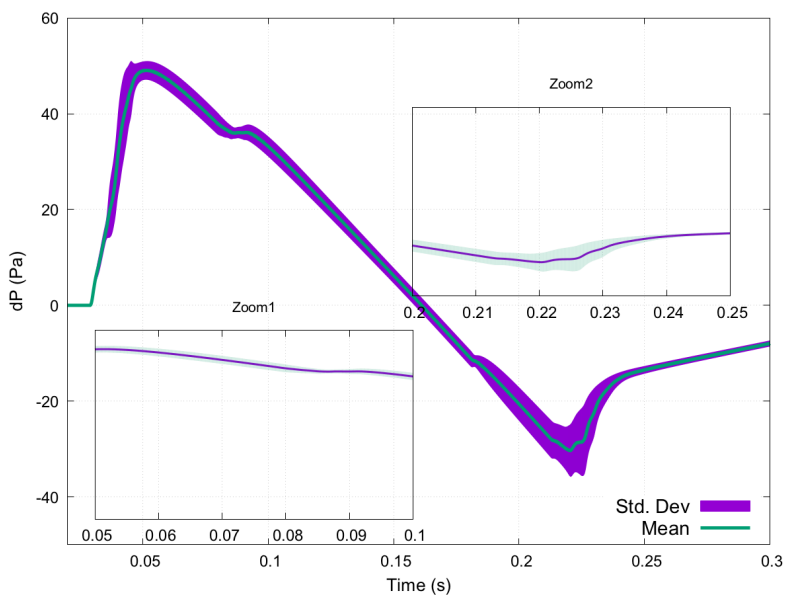

(b) Profile2 (Optional).

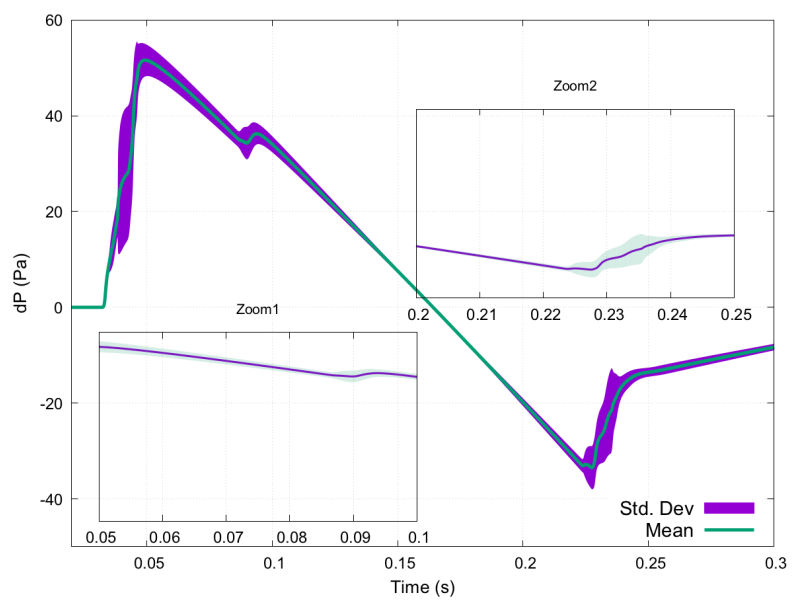

(d) Standard Profile w/ RH=70\% (Optional).

Figure 24. Mean and Standard deviation of Ground Signatures for LM1021 at Roll Angle $=30^{\circ}$ 


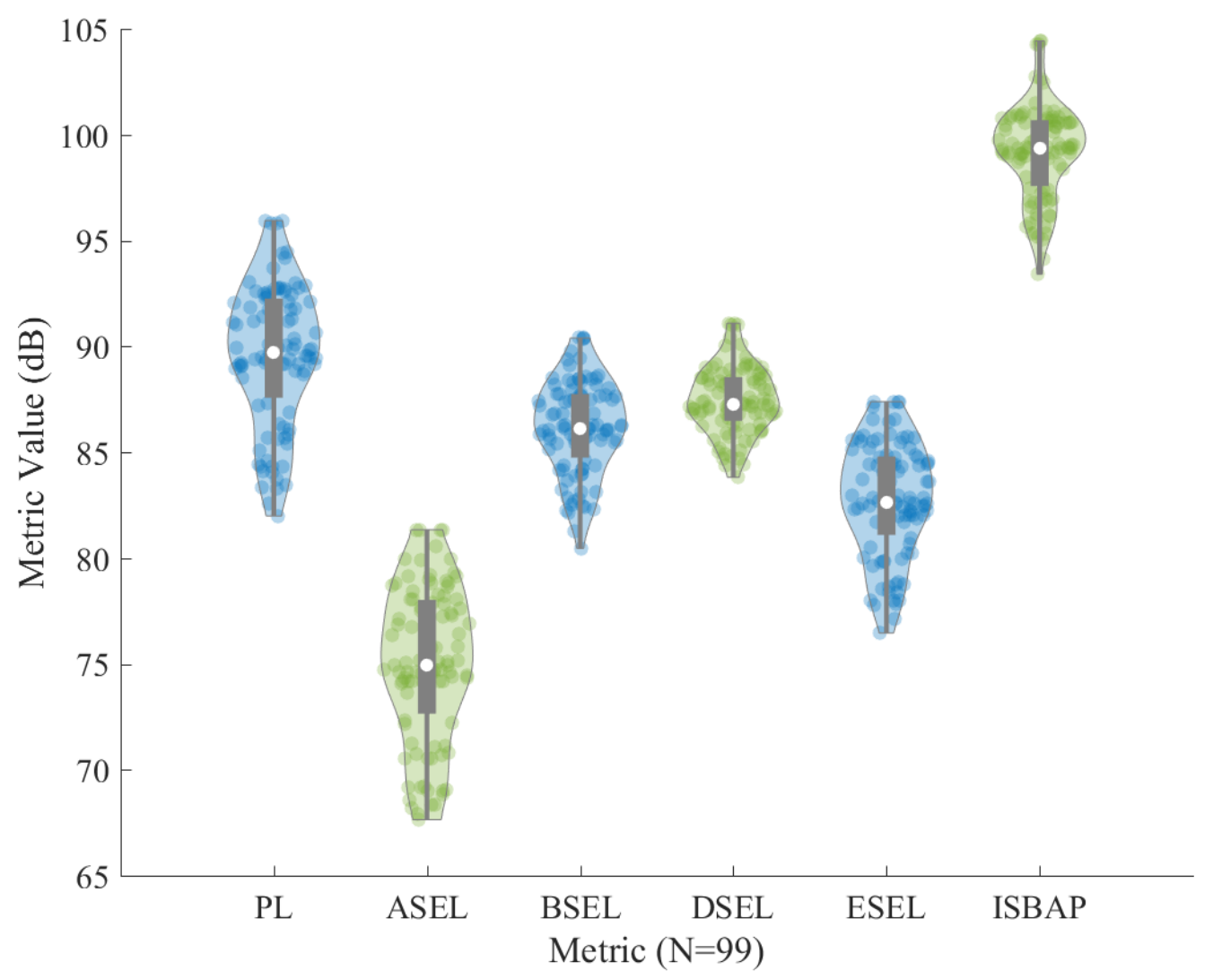

Figure 25. Violin plots of noise metrics for all LM1021 cases grouped together. 


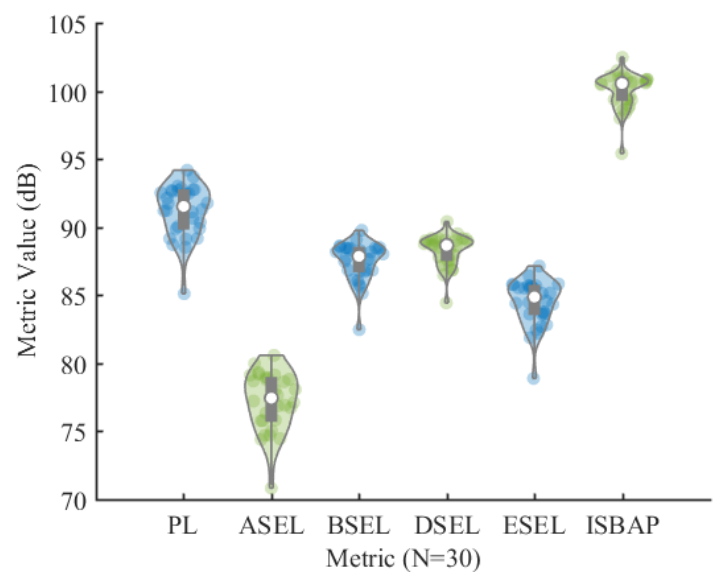

(a) Profile1 (Required).

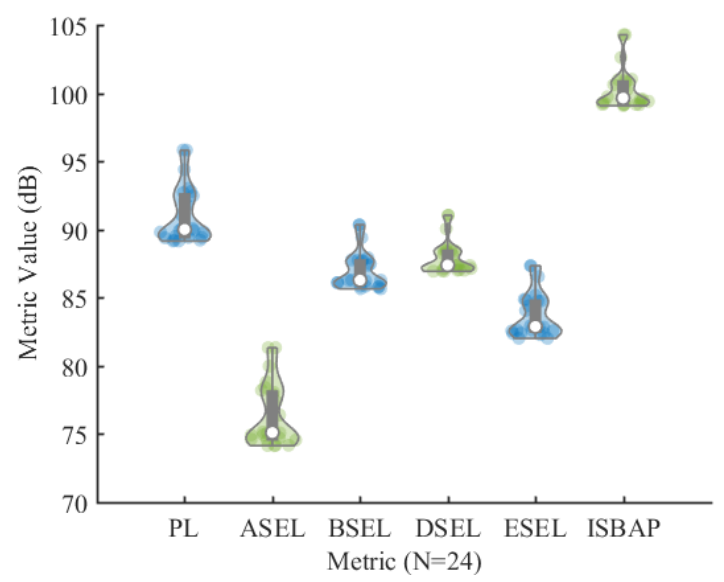

(c) Standard Profile (Optional).

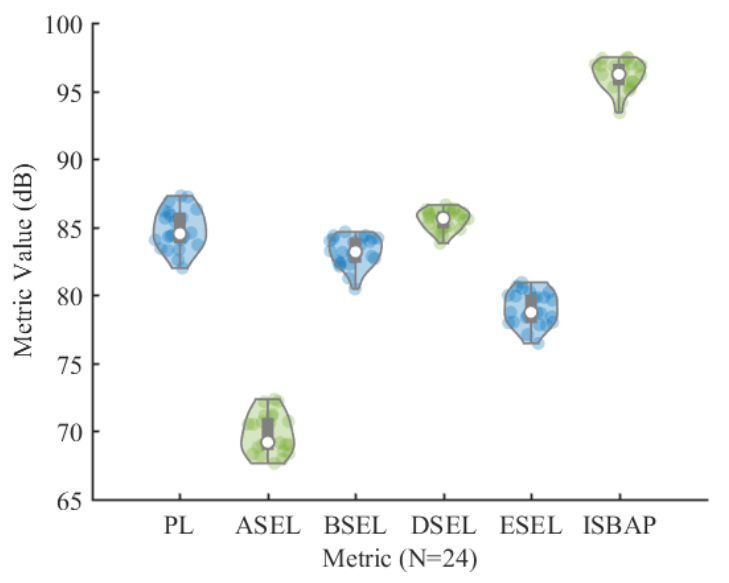

(b) Profile2 (Optional).

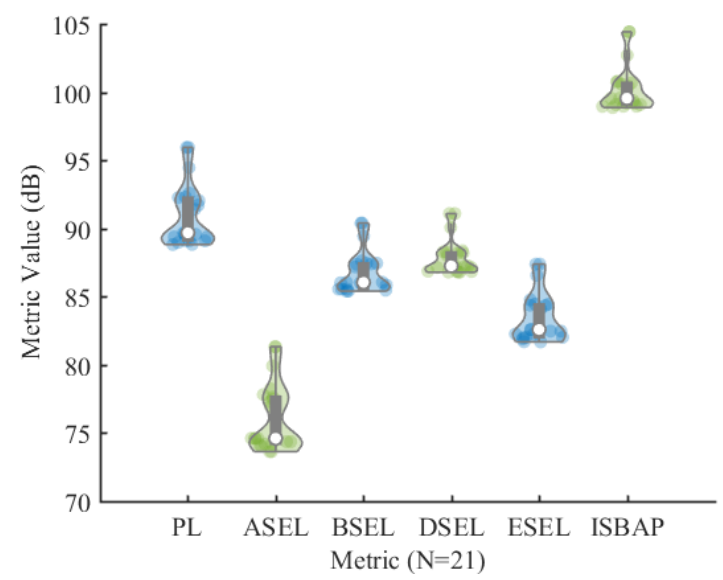

(d) Standard Profile w/ RH=70\% (Optional).

Figure 26. Violin plots of noise metrics for LM1021 separated by atmospheric conditions (all angles grouped together). 


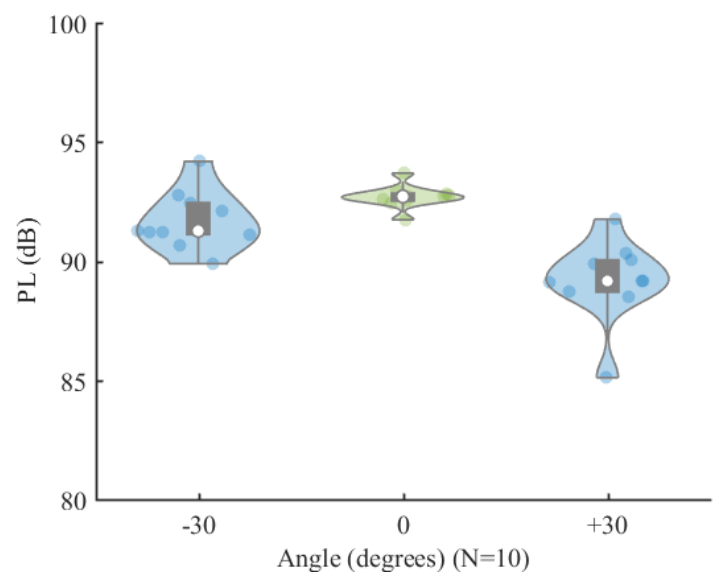

(a) Profile1 (Required).

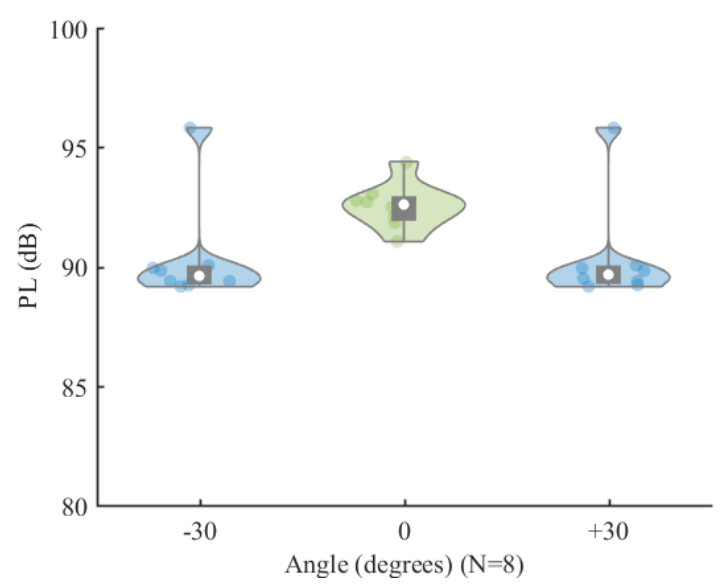

(c) Standard Profile (Optional).

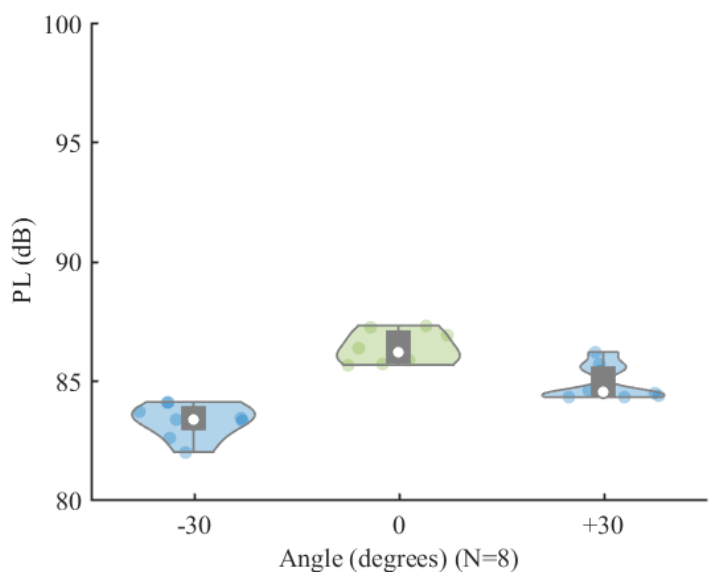

(b) Profile2 (Optional).

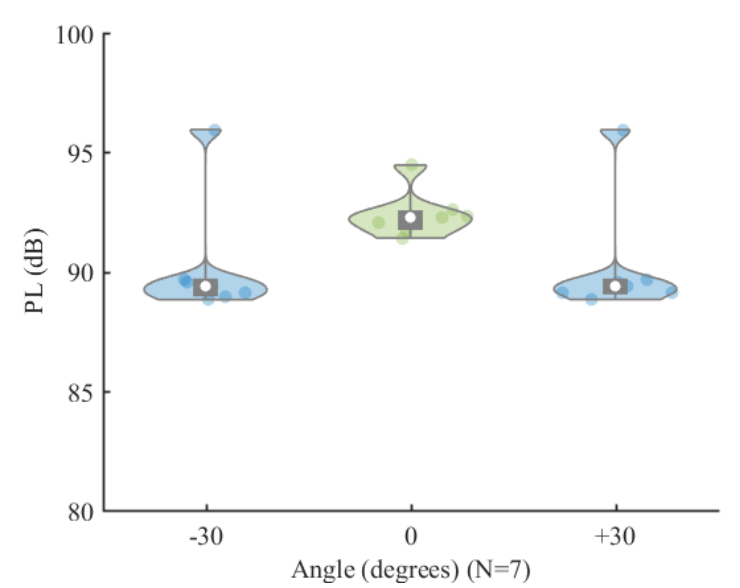

(d) Standard Profile w/ $\mathbf{R H}=\mathbf{7 0 \%}$ (Optional).

Figure 27. Violin plots of Perceived Level (PL) for LM1021 separated by atmospheric conditions and by angles. 


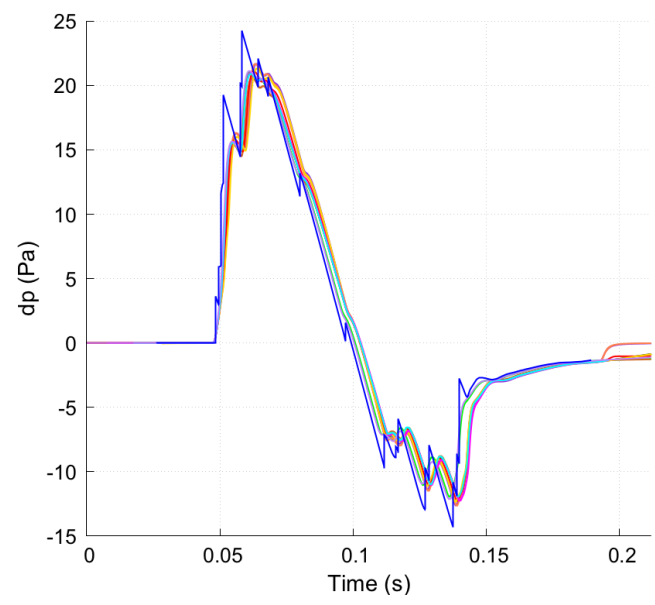

(a) Profile3 (Required).

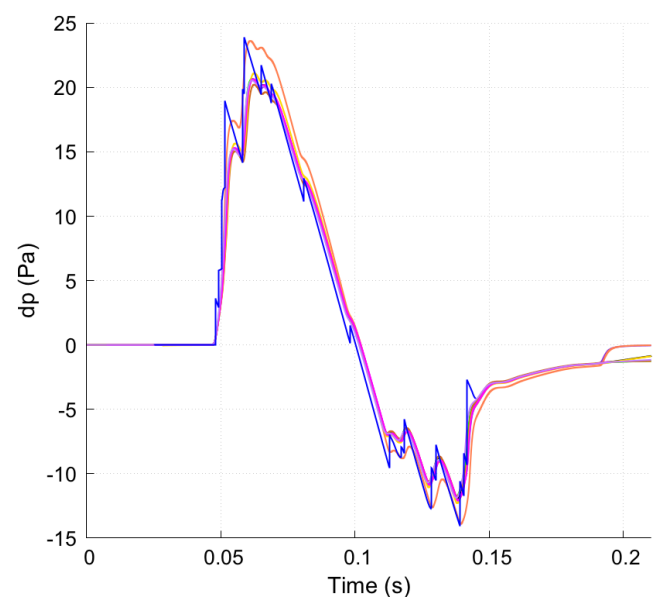

(c) Standard Profile (Optional).
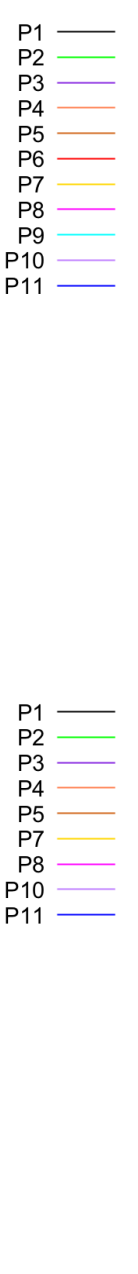

Figure 28. Ground Signatures for AXIBODY at Roll Angle $=0^{\circ}$

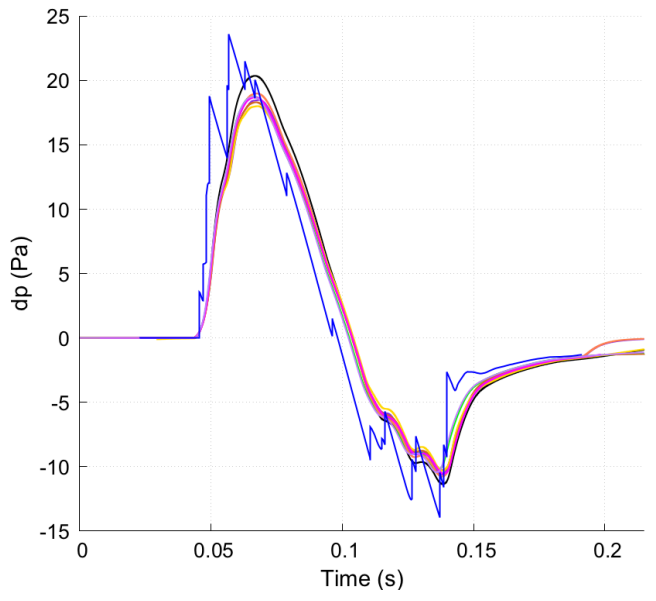

(b) Profile4 (Optional).

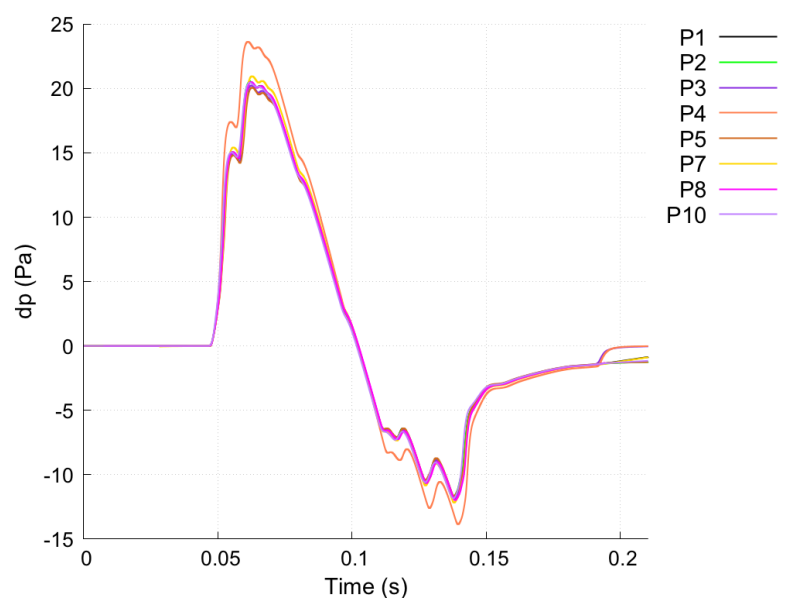

(d) Standard Profile w/ $\mathbf{R H}=\mathbf{7 0 \%}$ (Optional).

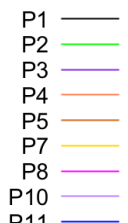

P11

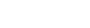




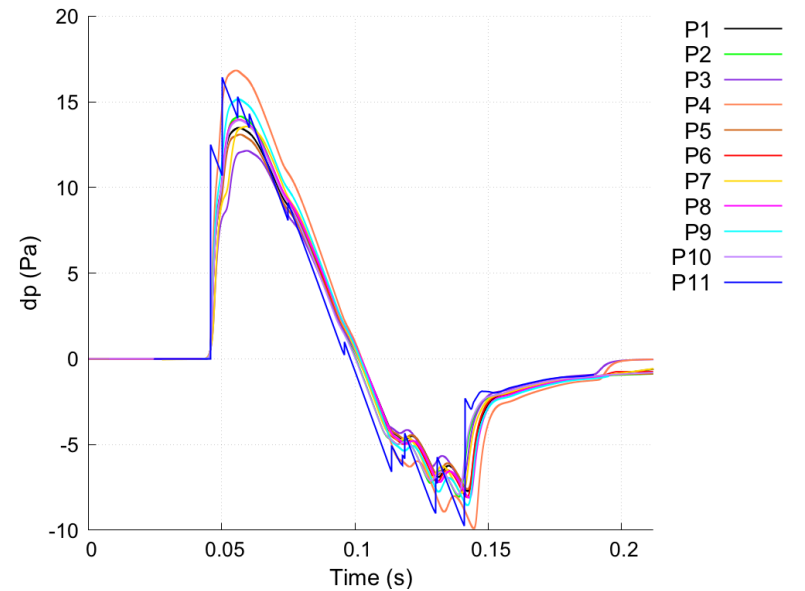

(a) Profile3 (Required).

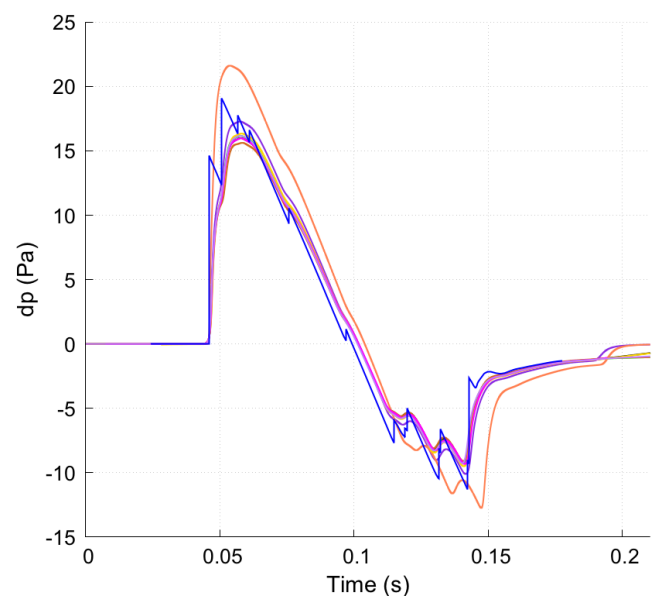

(c) Standard Profile (Optional).

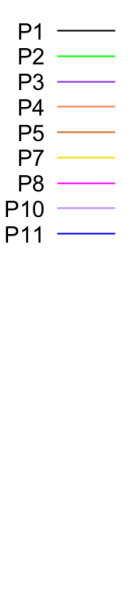

Figure 29. Ground Signatures for AXIBODY at Roll Angle $=-45^{\circ}$

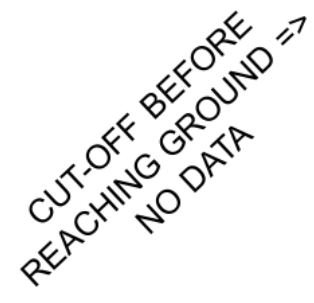

(b) Profile4 (Optional).

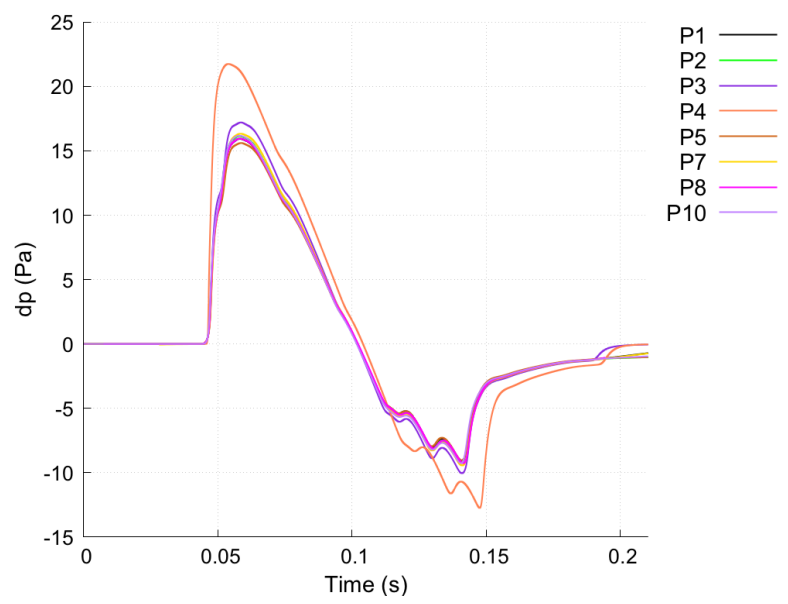

(d) Standard Profile w/ RH=70\% (Optional).

\section{9 of 48}




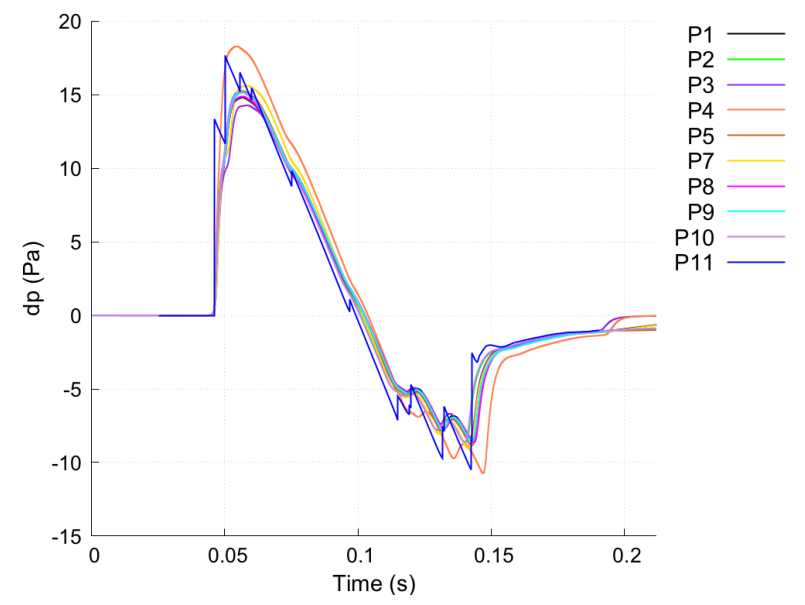

(a) Profile3 (Required).

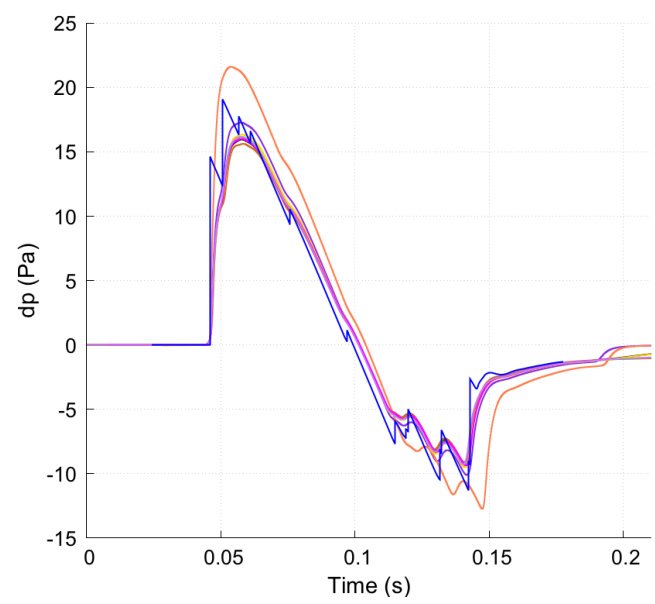

(c) Standard Profile (Optional).

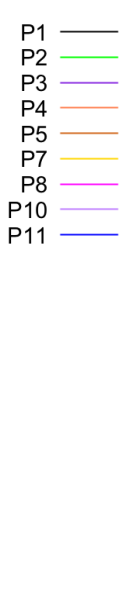

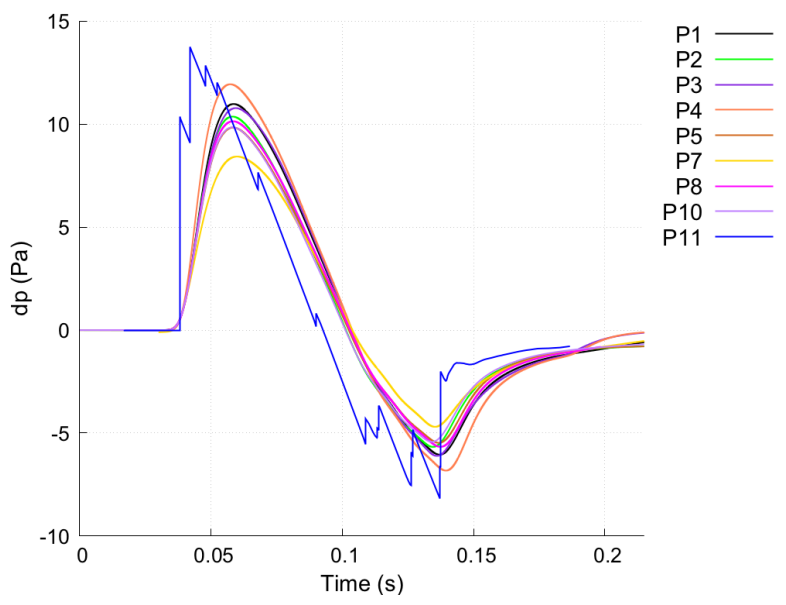

(b) Profile4 (Optional).

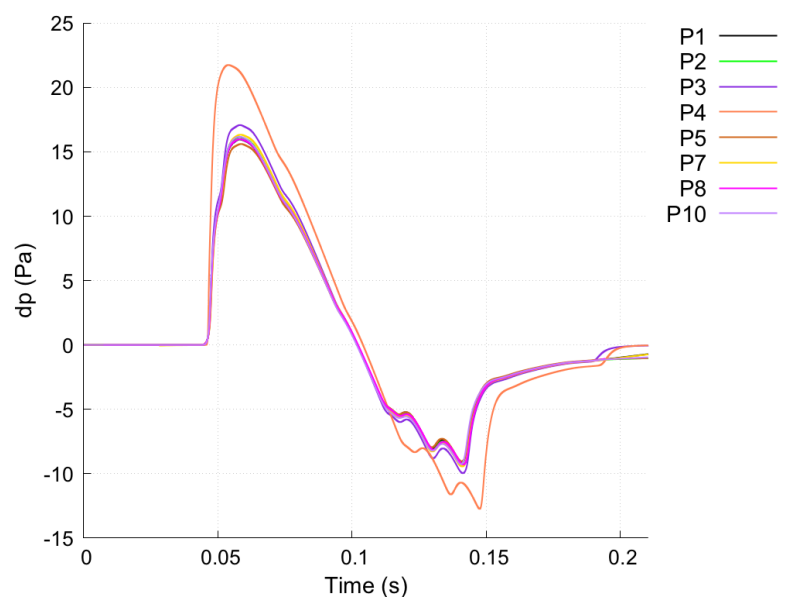

(d) Standard Profile w/ $\mathbf{R H}=\mathbf{7 0 \%}$ (Optional).

Figure 30. Ground Signatures for AXIBODY at Roll Angle $=45^{\circ}$ 


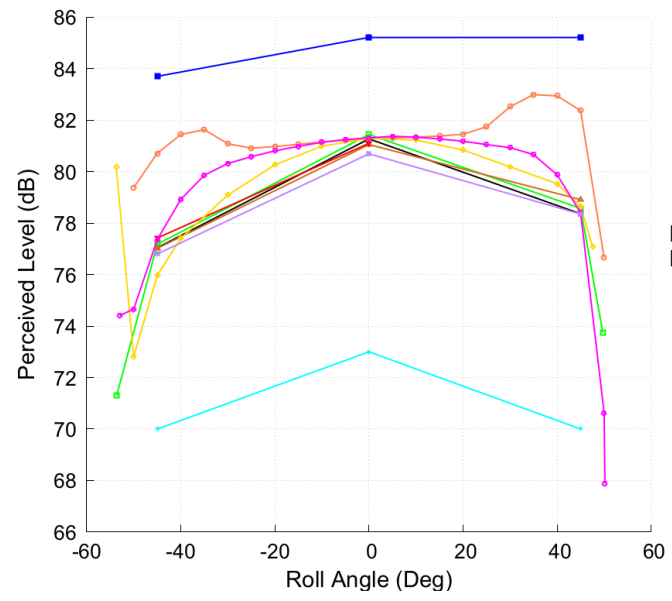

(a) Profile3 (Required).

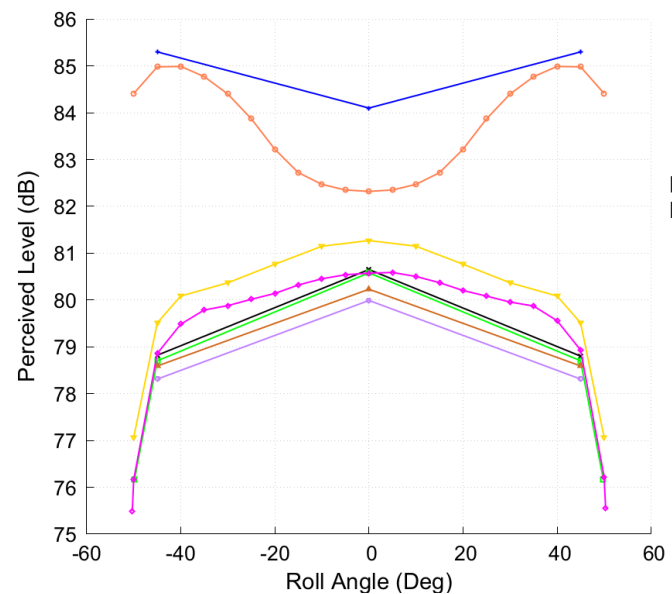

(c) Standard Profile (Optional).
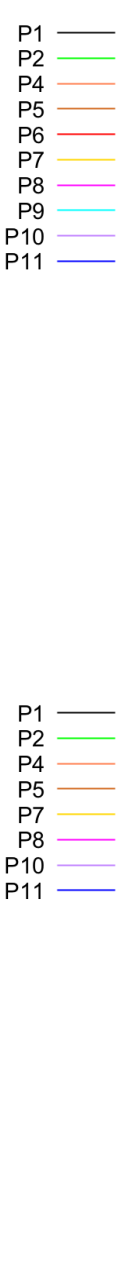

Figure 31. Submitted carpet loudness values for AXIBODY

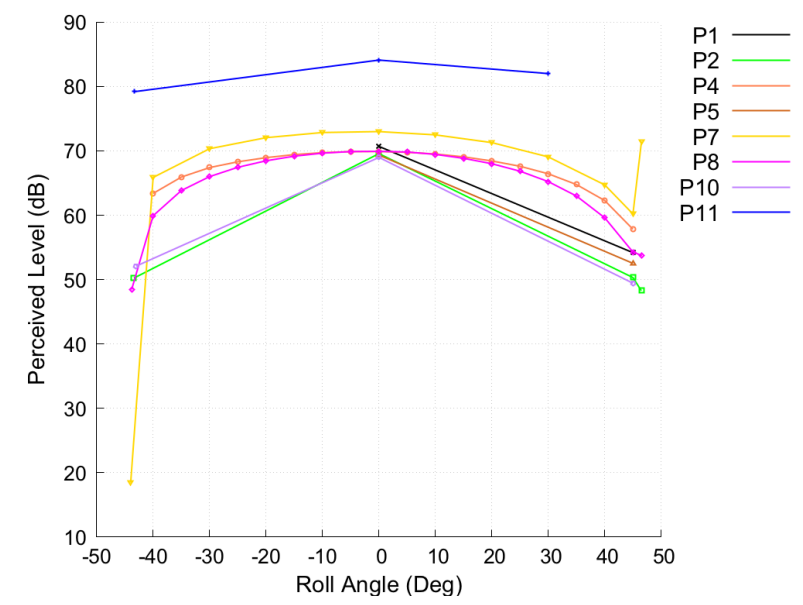

(b) Profile4 (Optional).

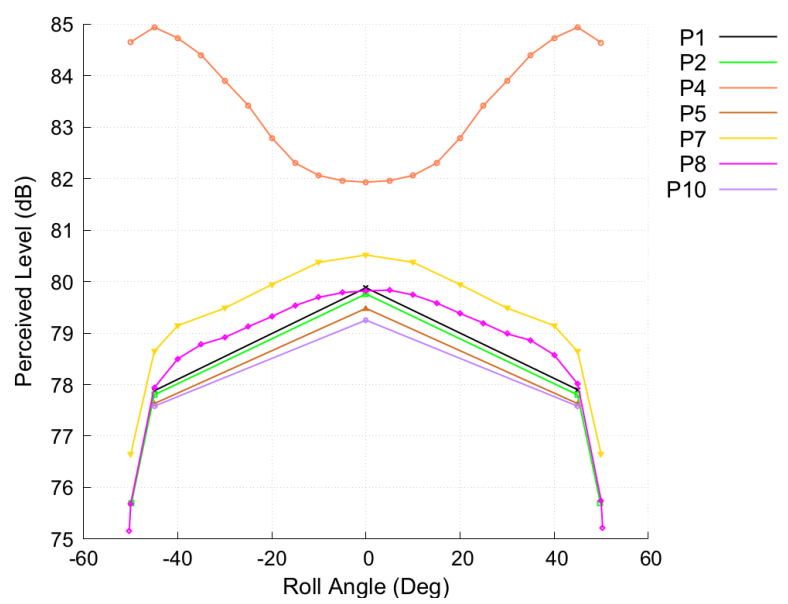

(d) Standard Profile w/ RH=70\% (Optional). 


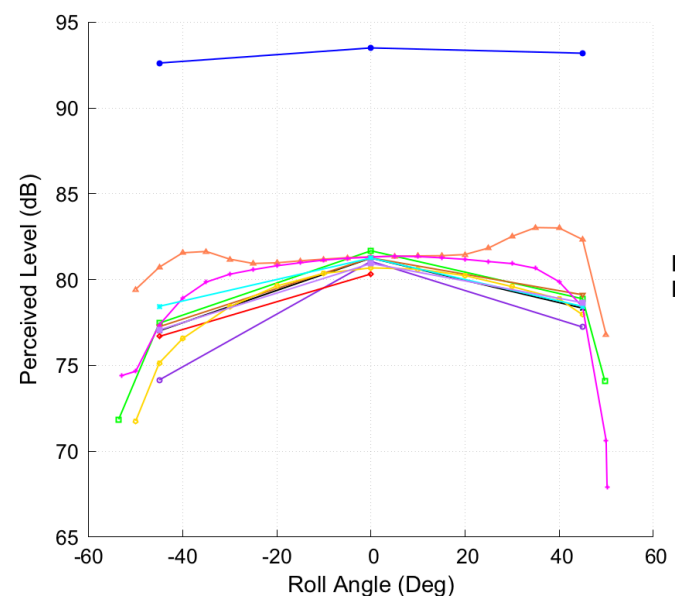

(a) Profile3 (Required).

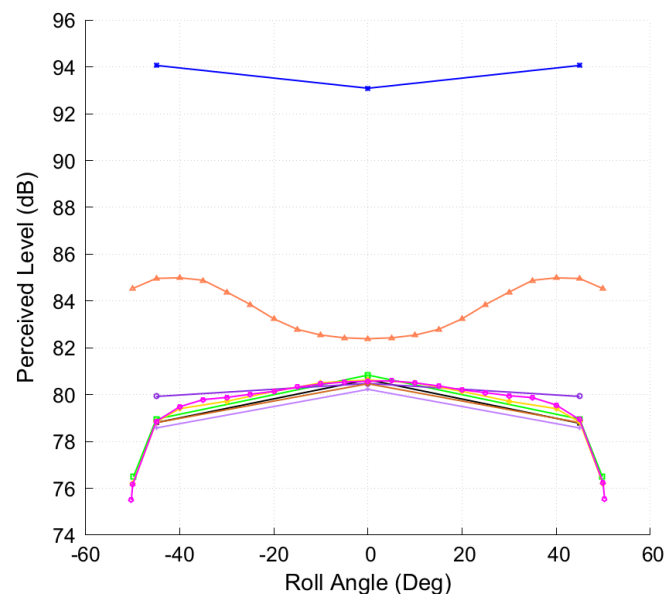

(c) Standard Profile (Optional).
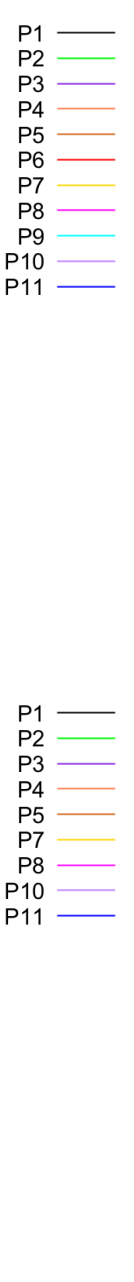

Figure 32. Calculated carpet loudness values for AXIBODY

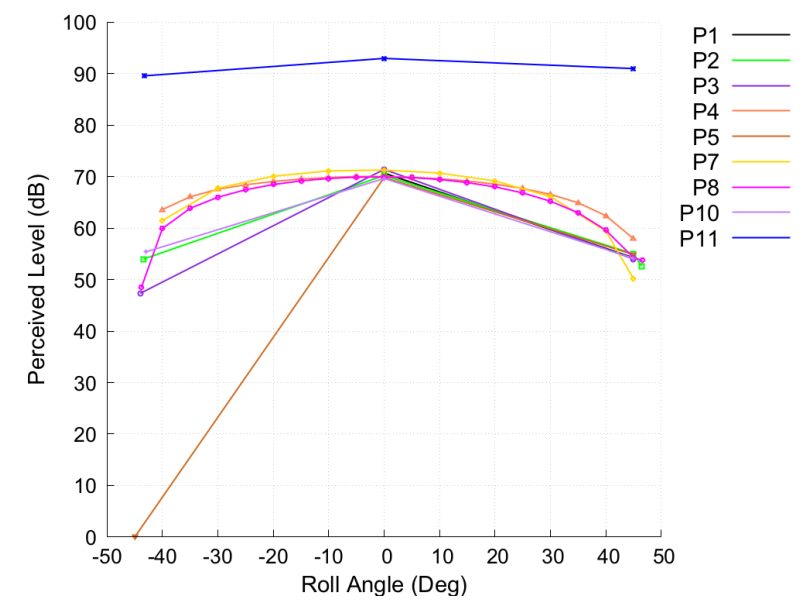

(b) Profile4 (Optional).

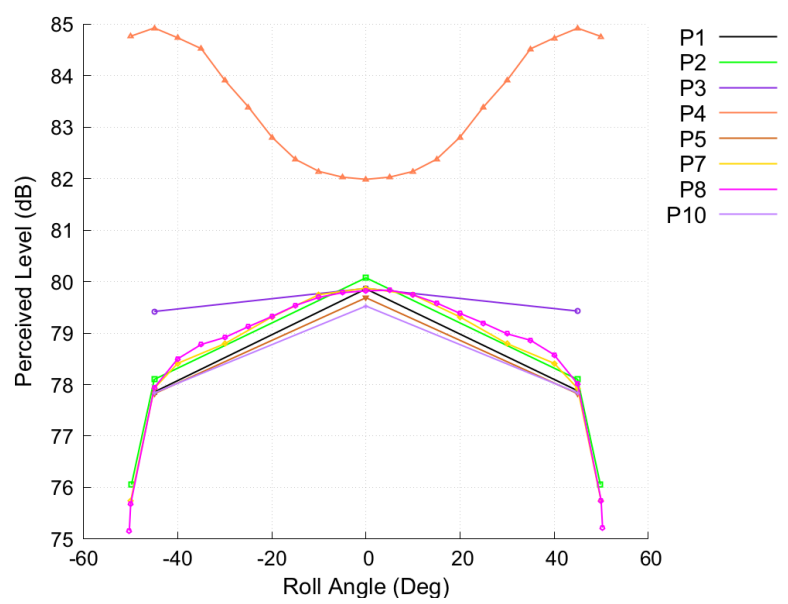

(d) Standard Profile w/ RH=70\% (Optional). 


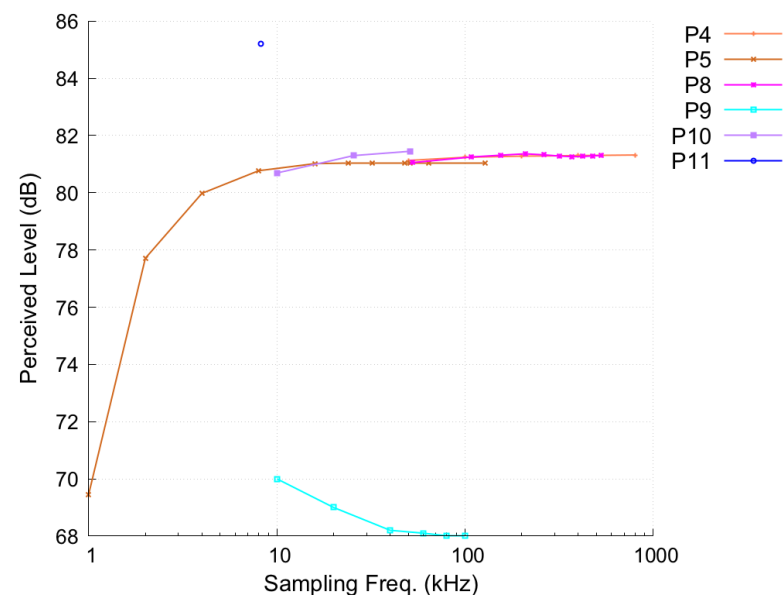

(a) Profile3 (Required).

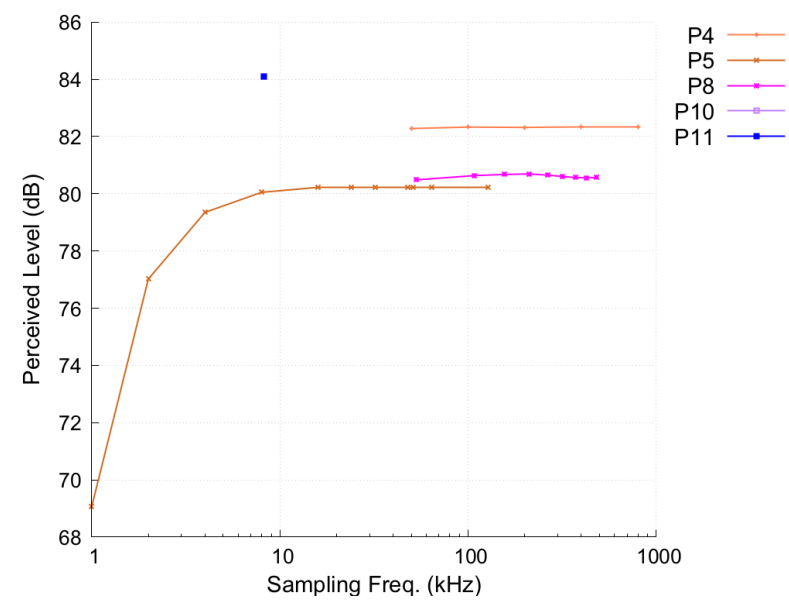

(c) Standard Profile (Optional).

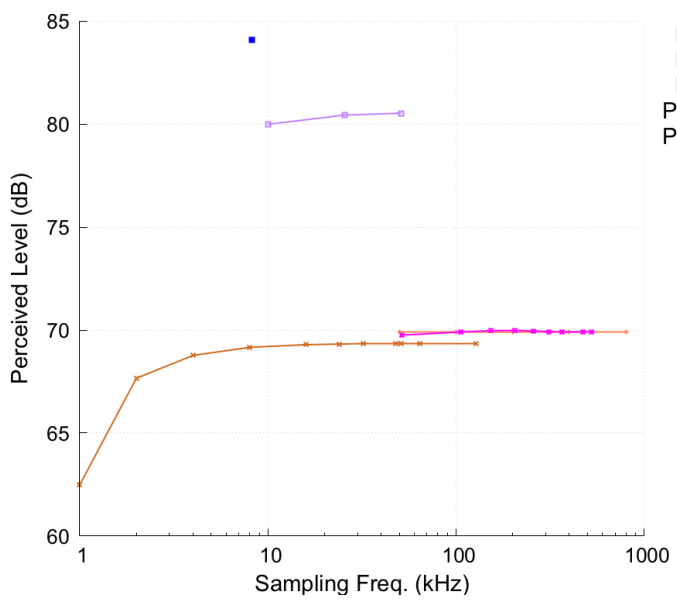

(b) Profile4 (Optional).

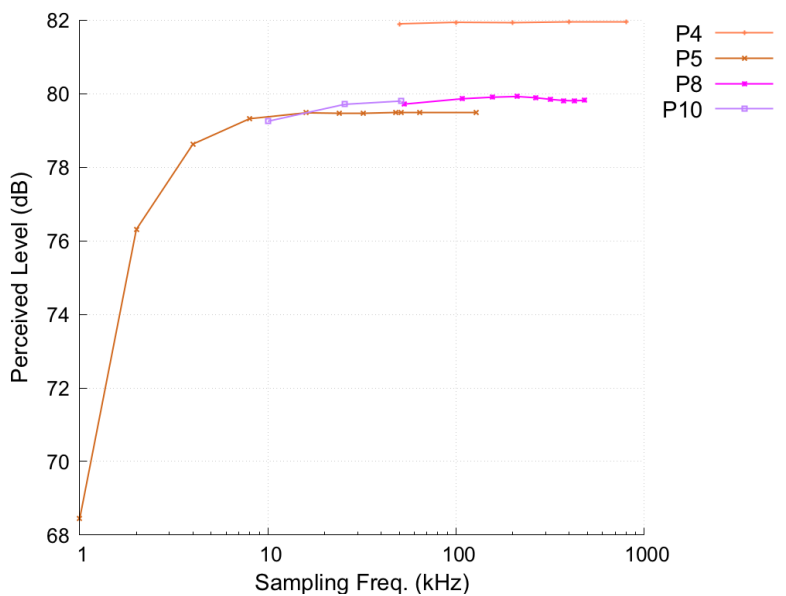

(d) Standard Profile w/ RH=70\% (Optional).

Figure 33. Loudness convergence for AXIBODY at Roll Angle $=0^{\circ}$ 


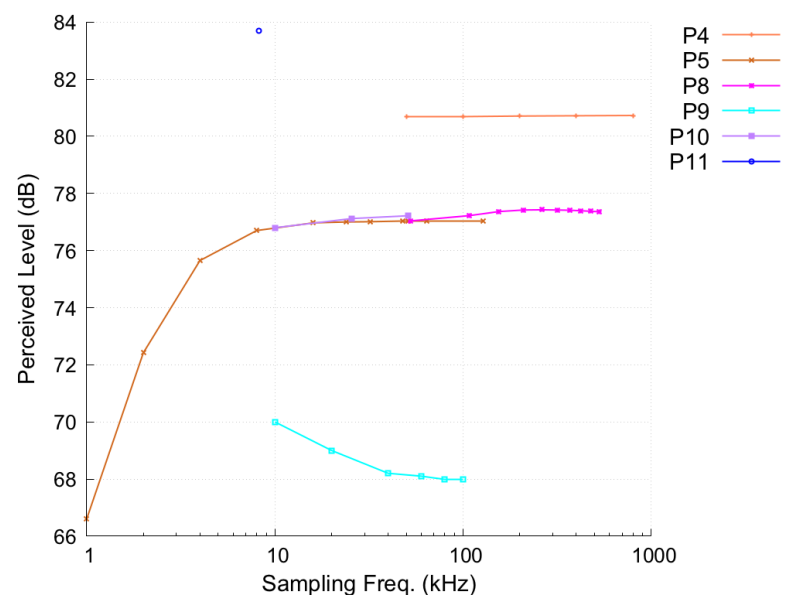

(a) Profile3 (Required).

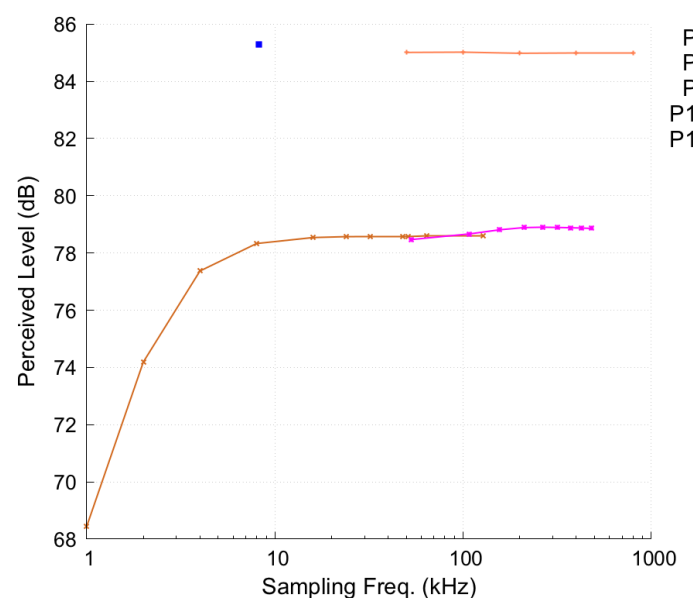

(c) Standard Profile (Optional).

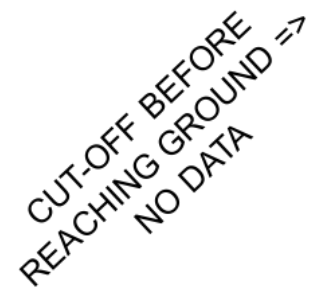

(b) Profile4 (Optional).
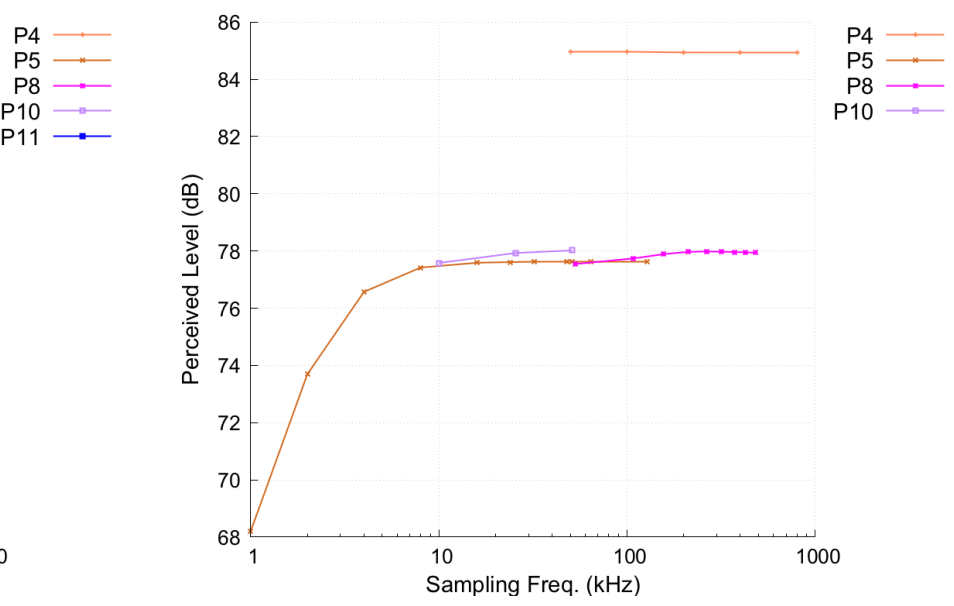

(d) Standard Profile w/ RH=70\% (Optional).

Figure 34. Loudness convergence for AXIBODY at Roll Angle $=-45^{\circ}$ 


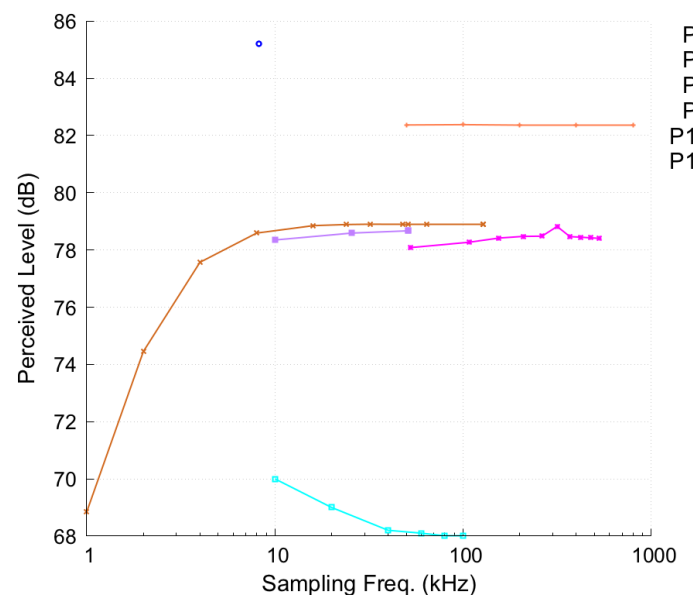

(a) Profile3 (Required).

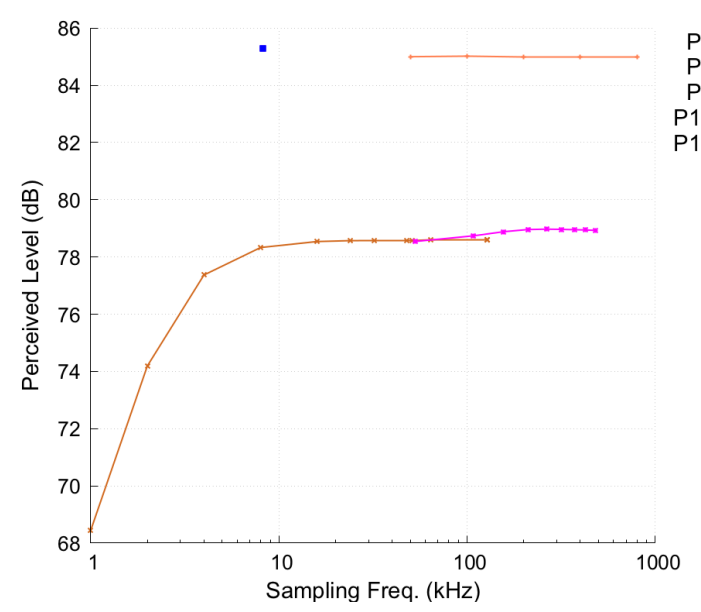

(c) Standard Profile (Optional).

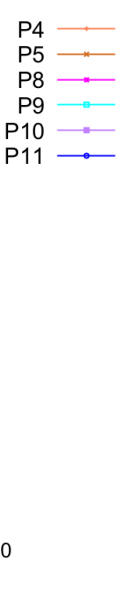

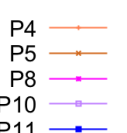

Figure 35. Loudness convergence for AXIBODY at Roll Angle $=45^{\circ}$

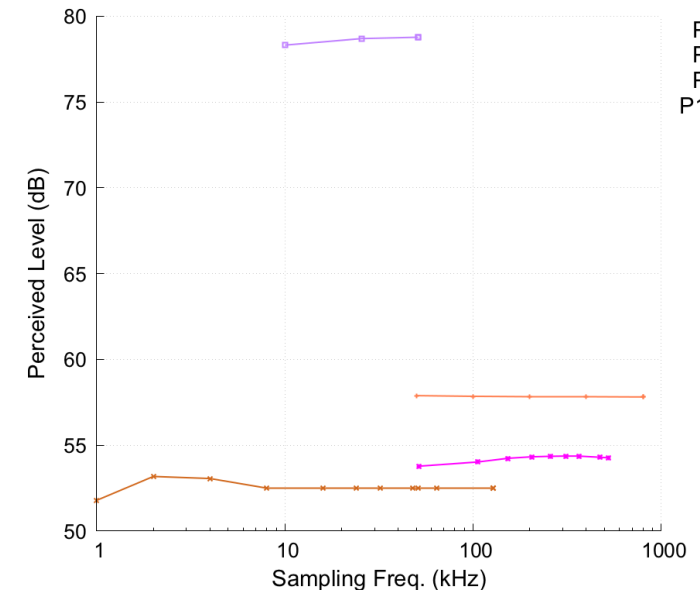

(b) Profile4 (Optional).

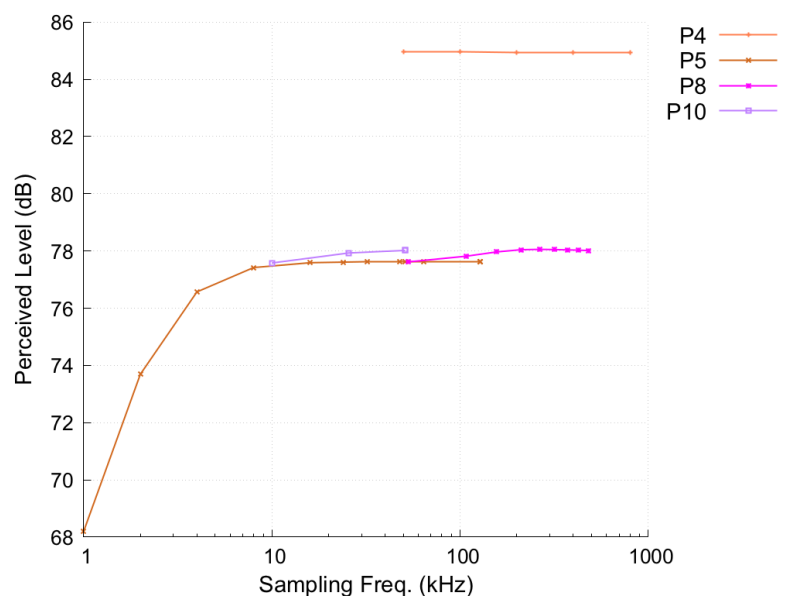

(d) Standard Profile w/ RH=70\% (Optional).

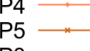

$\mathrm{P} 10 \longrightarrow$

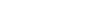




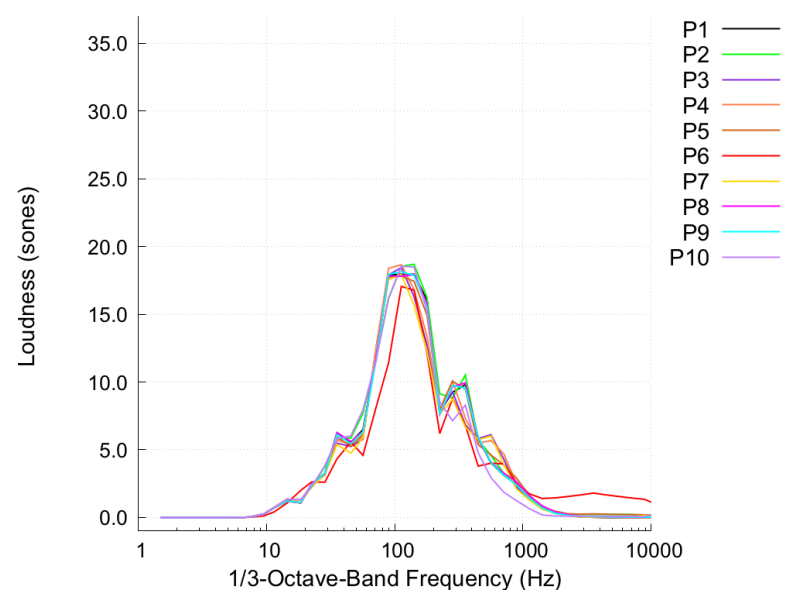

(a) Profile3 (Required).

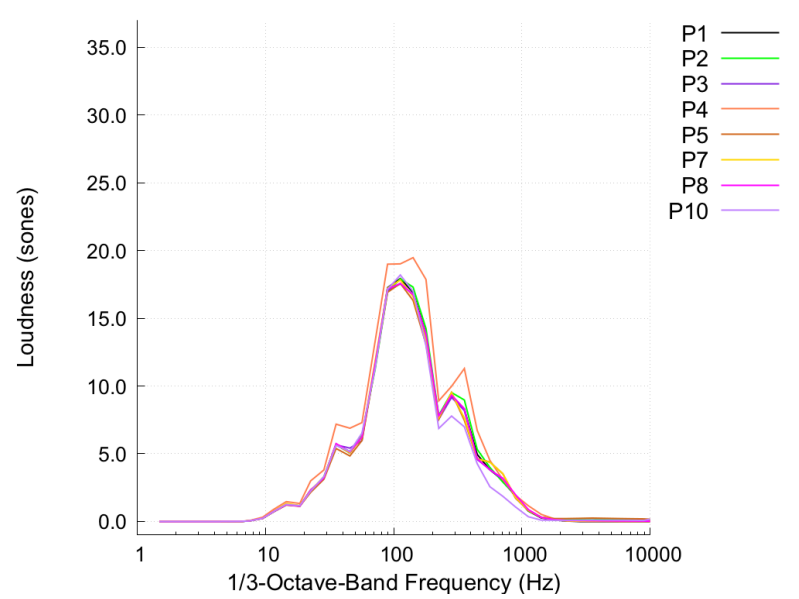

(c) Standard Profile (Optional).

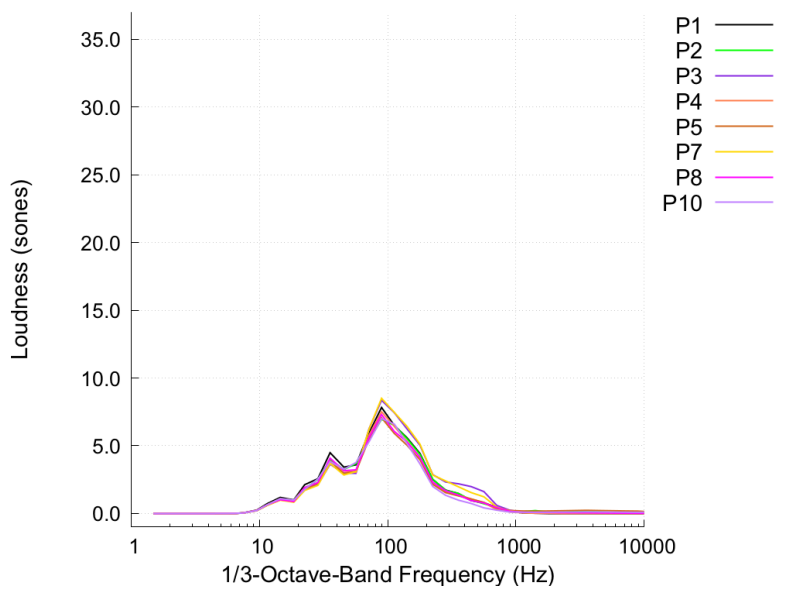

(b) Profile4 (Optional).

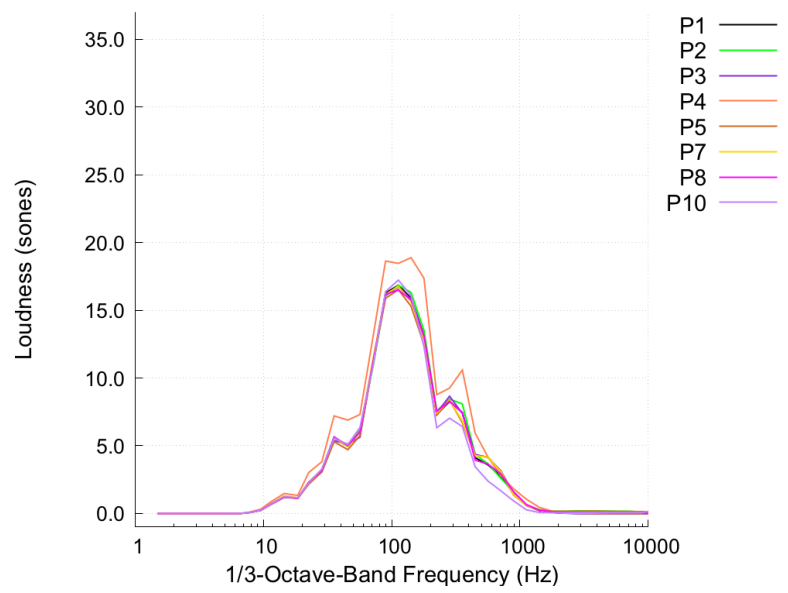

(d) Standard Profile w/ $\mathbf{R H}=\mathbf{7 0 \%}$ (Optional).

Figure 36. Frequency spectra of Ground Signatures for AXIBODY at Roll Angle $=0^{\circ}$ 


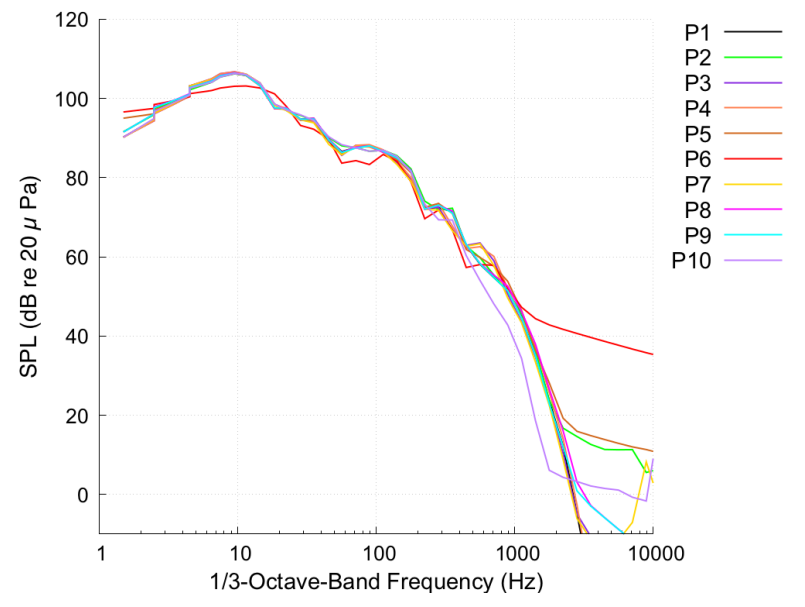

(a) Profile3 (Required).

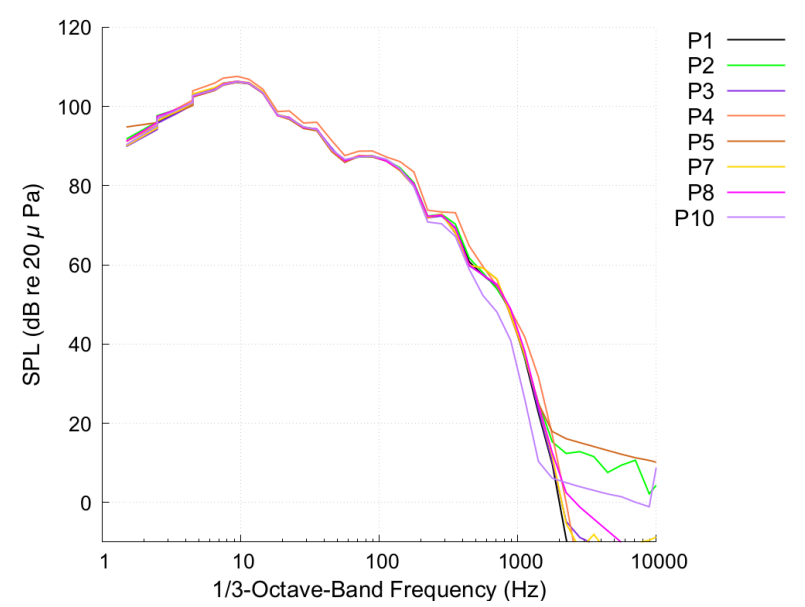

(c) Standard Profile (Optional).

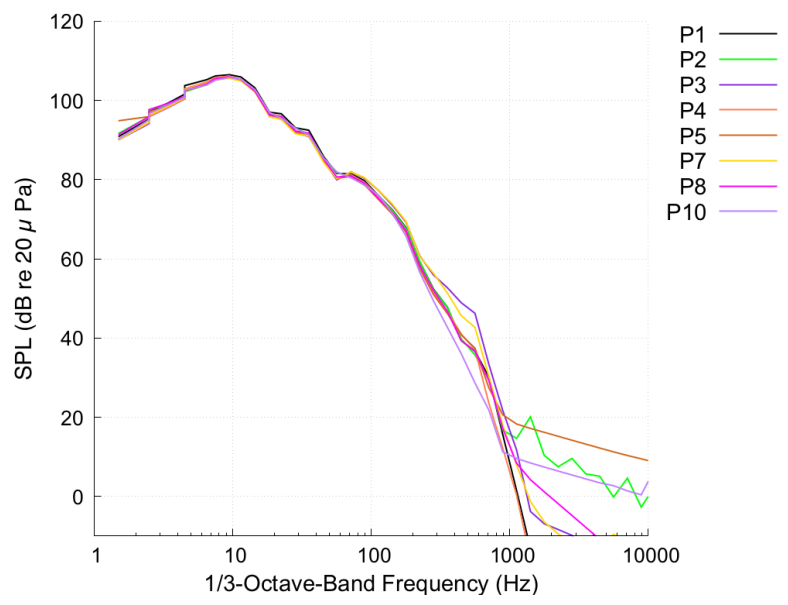

(b) Profile4 (Optional).

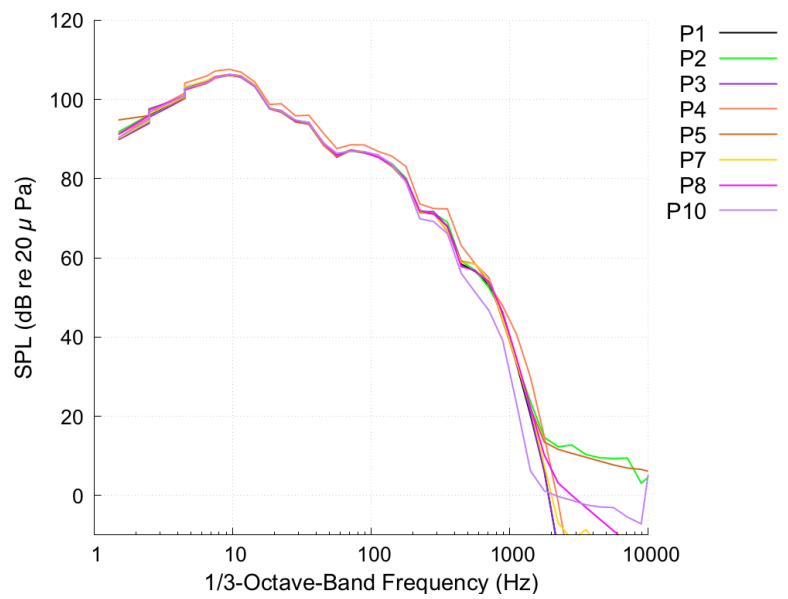

(d) Standard Profile w/ RH=70\% (Optional).

Figure 37. Frequency spectra of Ground Signatures for AXIBODY at Roll Angle $=0^{\circ}$ 


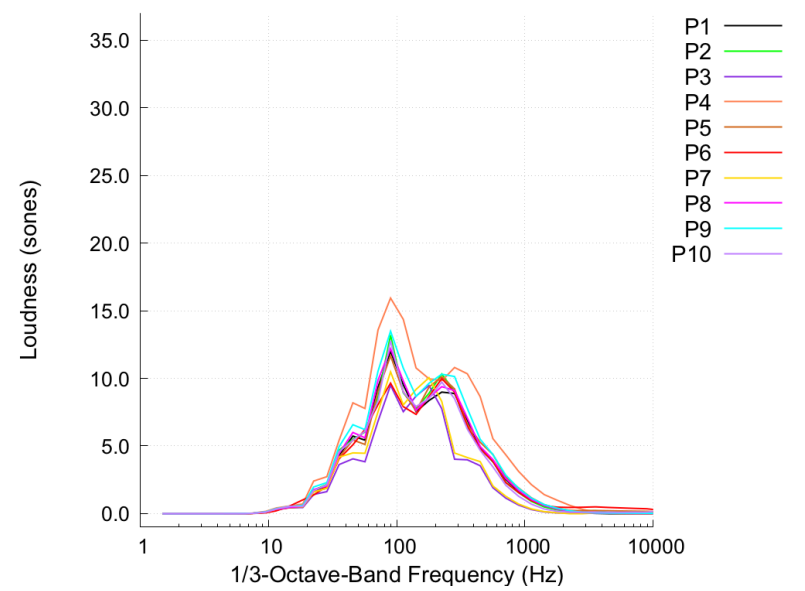

(a) Profile3 (Required).

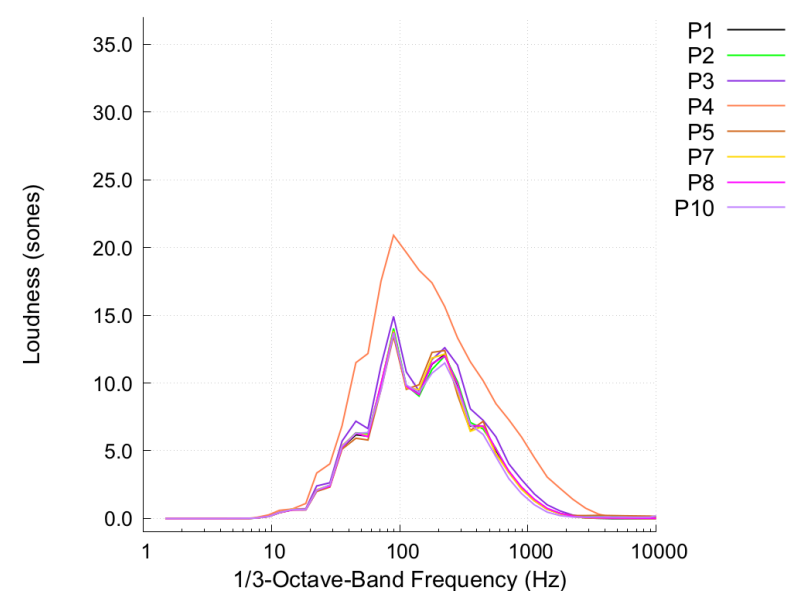

(c) Standard Profile (Optional).

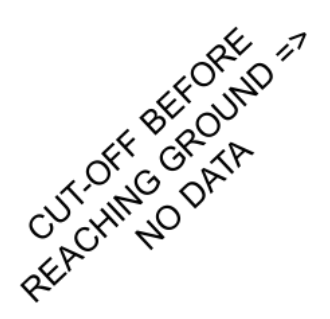

(b) Profile4 (Optional).

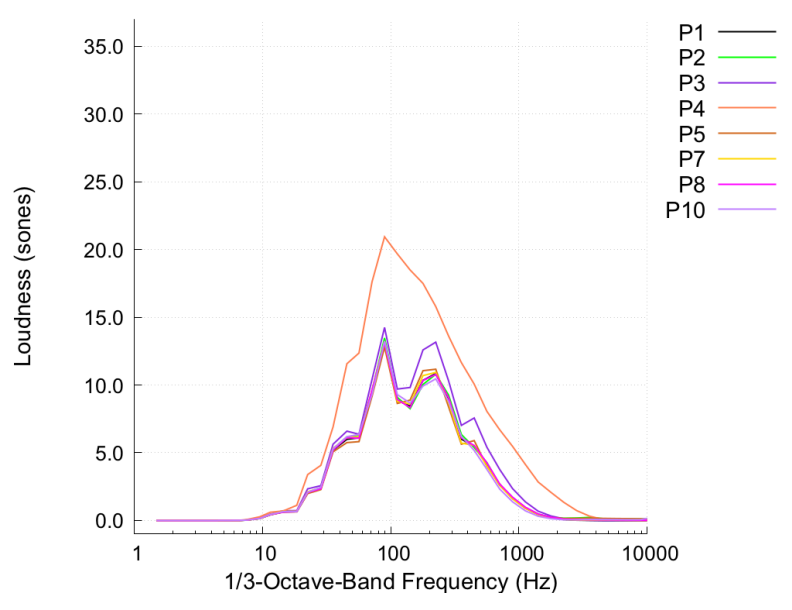

(d) Standard Profile w/ RH=70\% (Optional).

Figure 38. Frequency spectra of Ground Signatures for AXIBODY at Roll Angle $=-45^{\circ}$ 


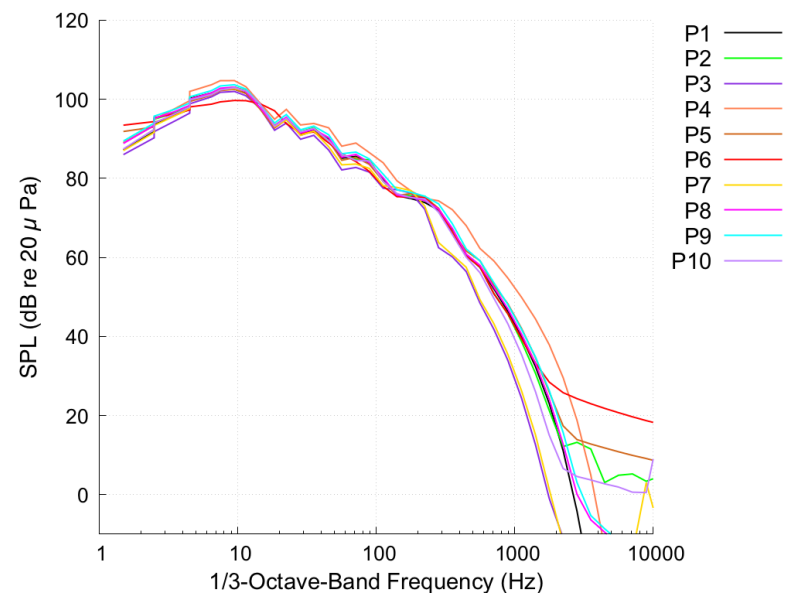

(a) Profile3 (Required).

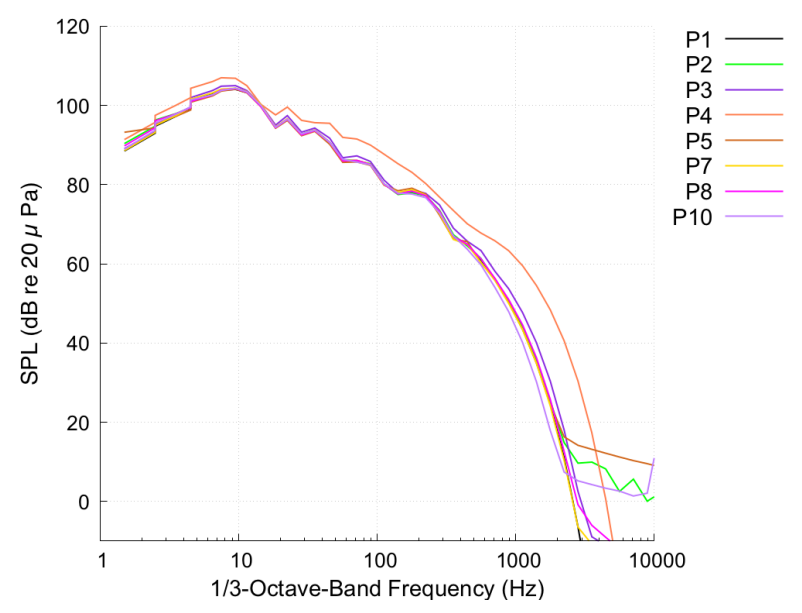

(c) Standard Profile (Optional).

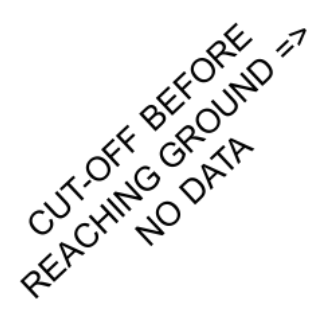

(b) Profile4 (Optional).

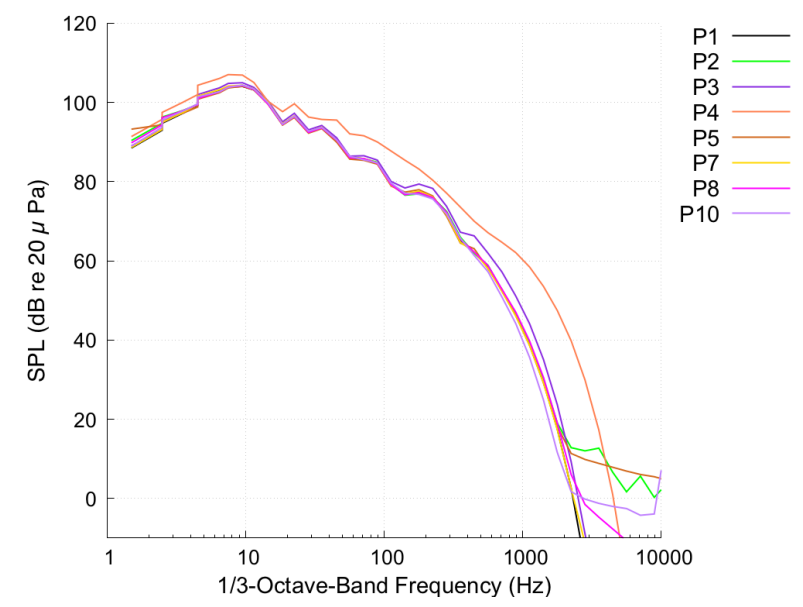

(d) Standard Profile w/ RH=70\% (Optional).

Figure 39. Frequency spectra of Ground Signatures for AXIBODY at Roll Angle $=-45^{\circ}$ 


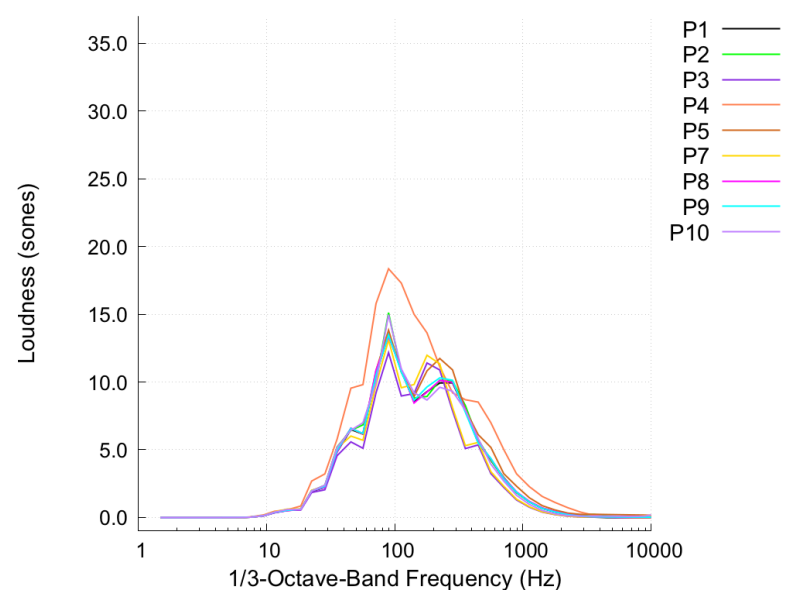

(a) Profile3 (Required).

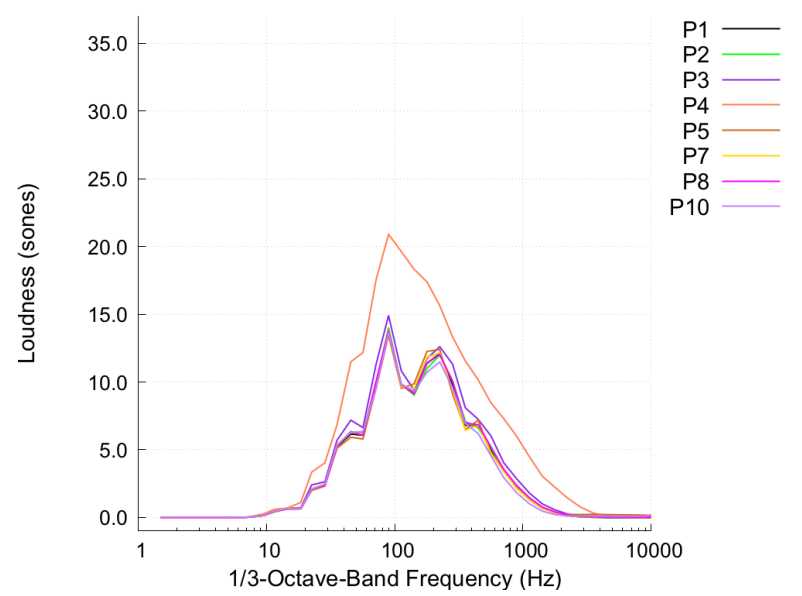

(c) Standard Profile (Optional).

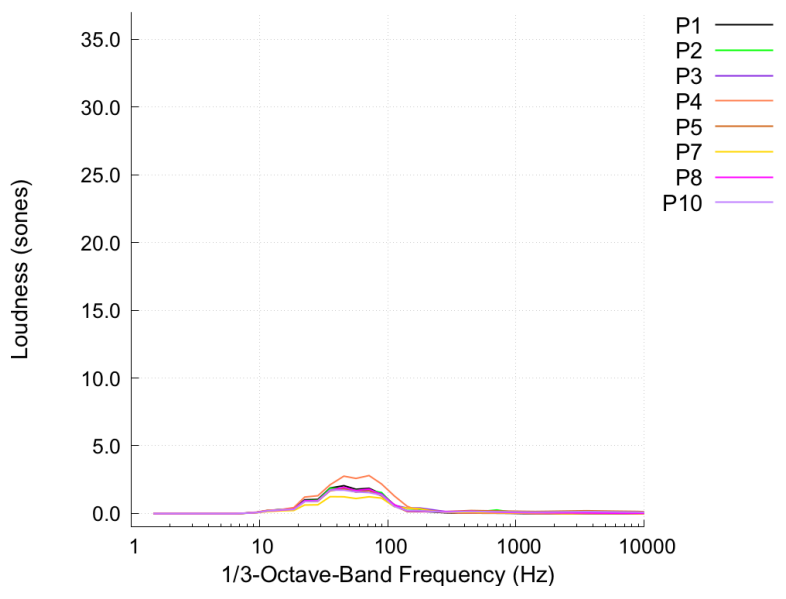

(b) Profile4 (Optional).

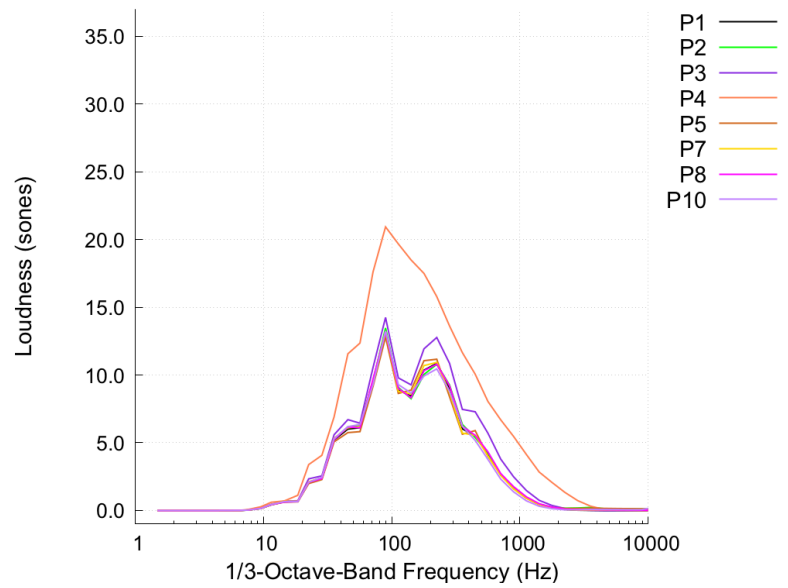

(d) Standard Profile w/ RH=70\% (Optional).

Figure 40. Frequency spectra of Ground Signatures for AXIBODY at Roll Angle $=45^{\circ}$ 


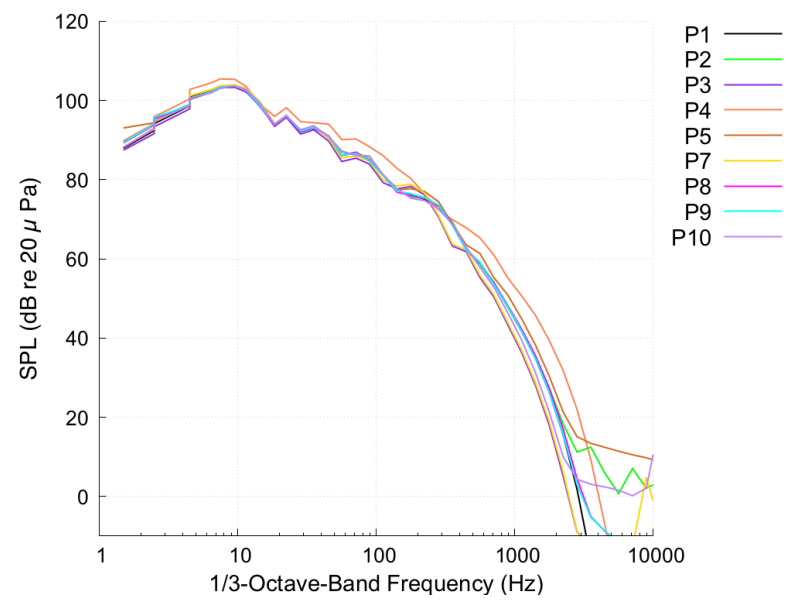

(a) Profile3 (Required).

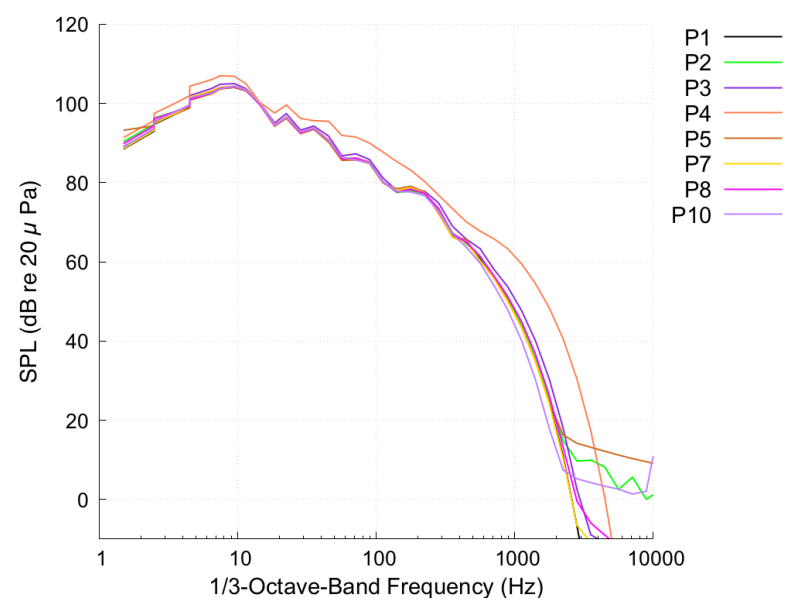

(c) Standard Profile (Optional).

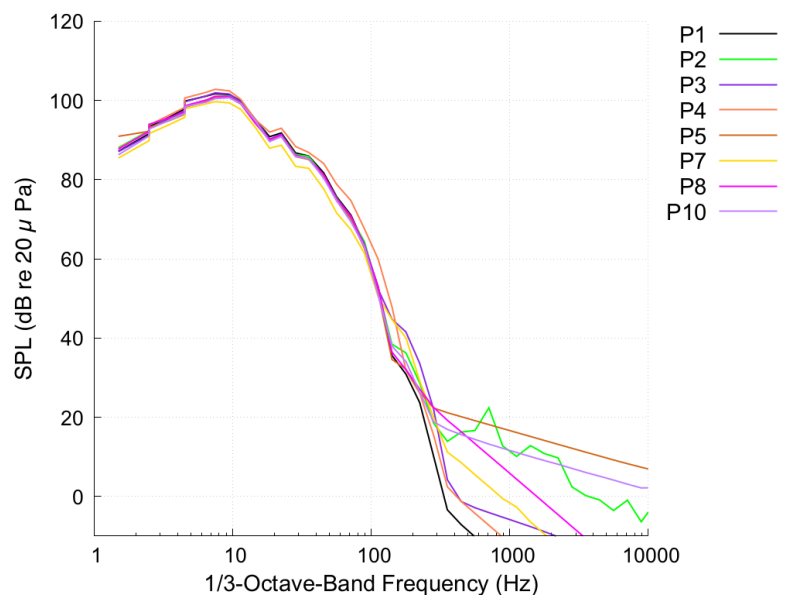

(b) Profile4 (Optional).

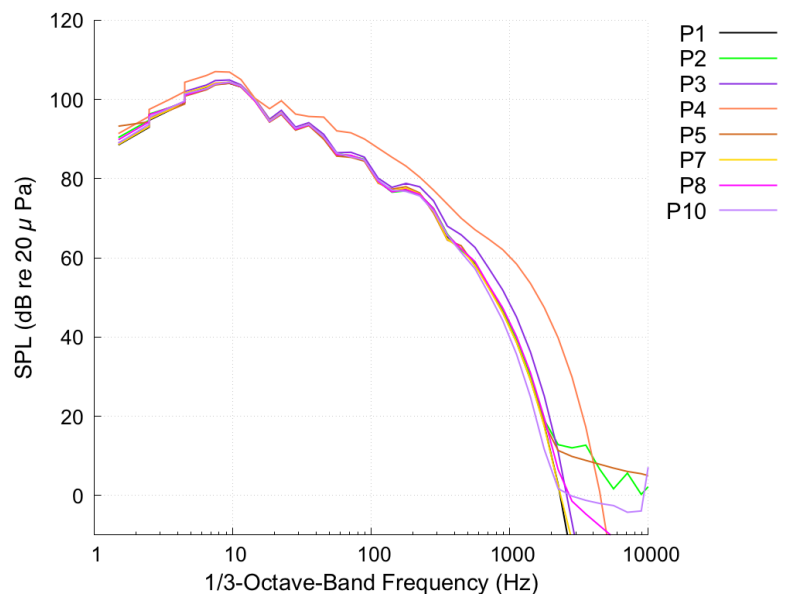

(d) Standard Profile w/ RH=70\% (Optional).

Figure 41. Frequency spectra of Ground Signatures for AXIBODY at Roll Angle $=45^{\circ}$ 


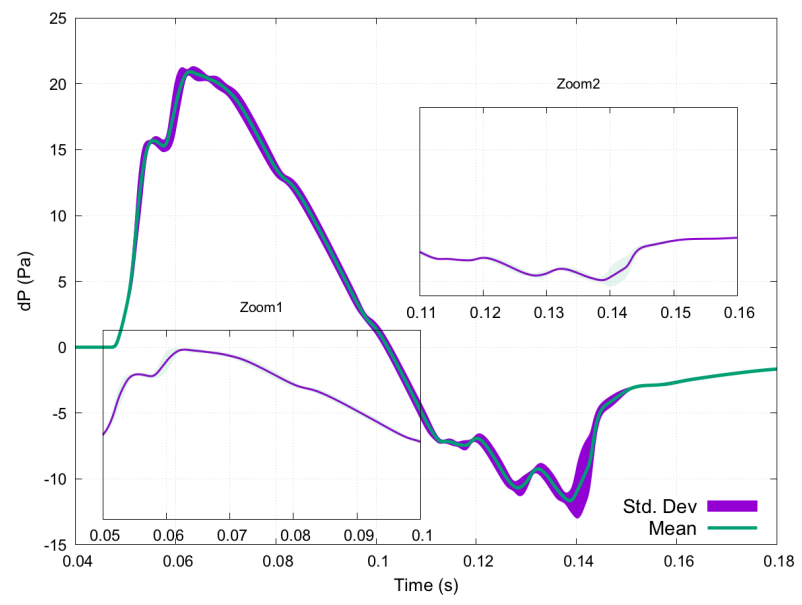

(a) Profile3 (Required).

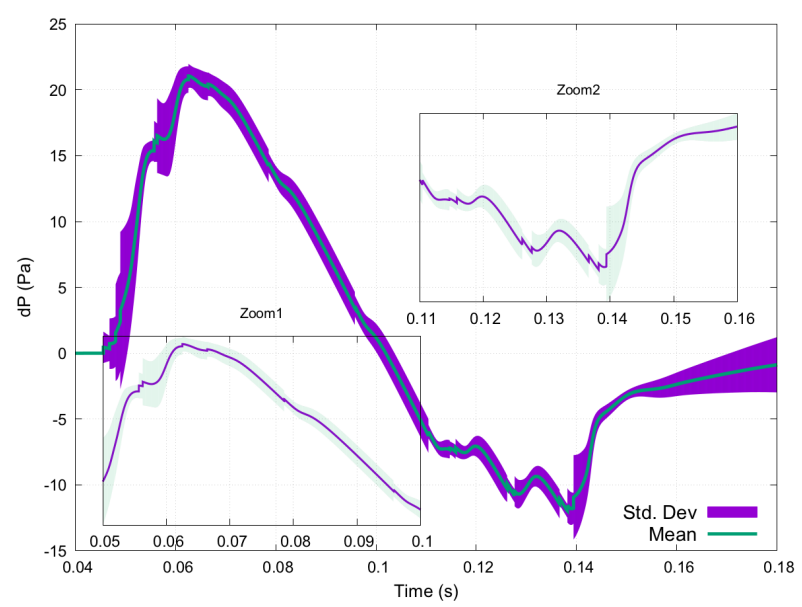

(c) Standard Profile (Optional).

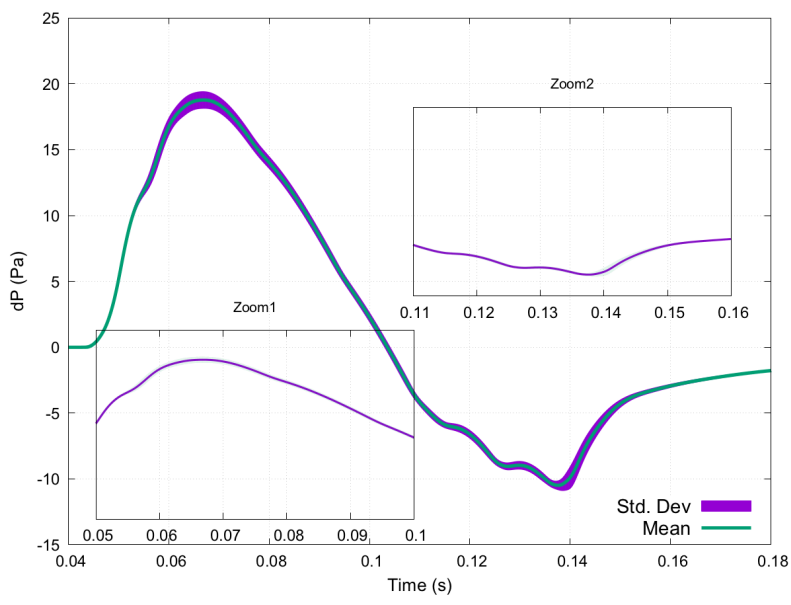

(b) Profile4 (Optional).

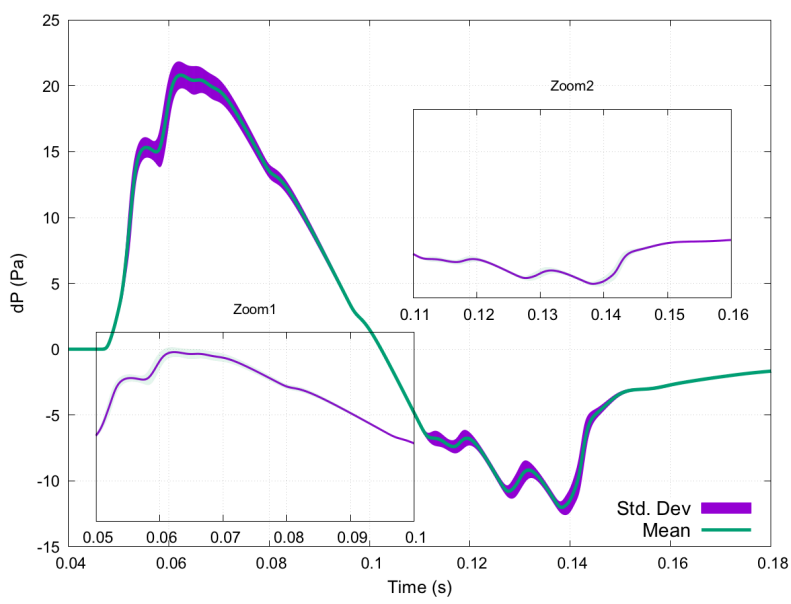

(d) Standard Profile w/ RH=70\% (Optional).

Figure 42. Mean and Standard deviation of Ground Signatures for AXIBODY at Roll Angle $=0^{\circ}$ 


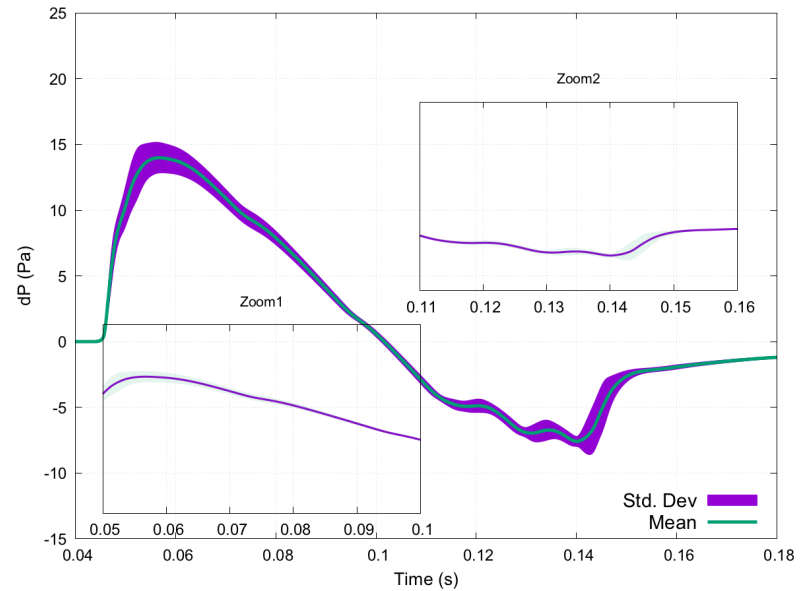

(a) Profile3 (Required).

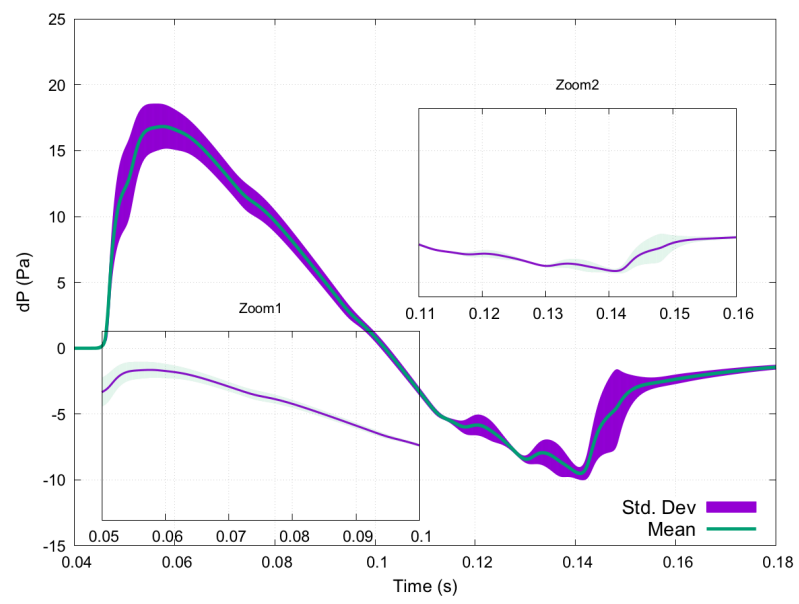

(c) Standard Profile (Optional).

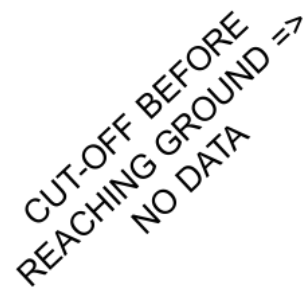

(b) Profile4 (Optional).

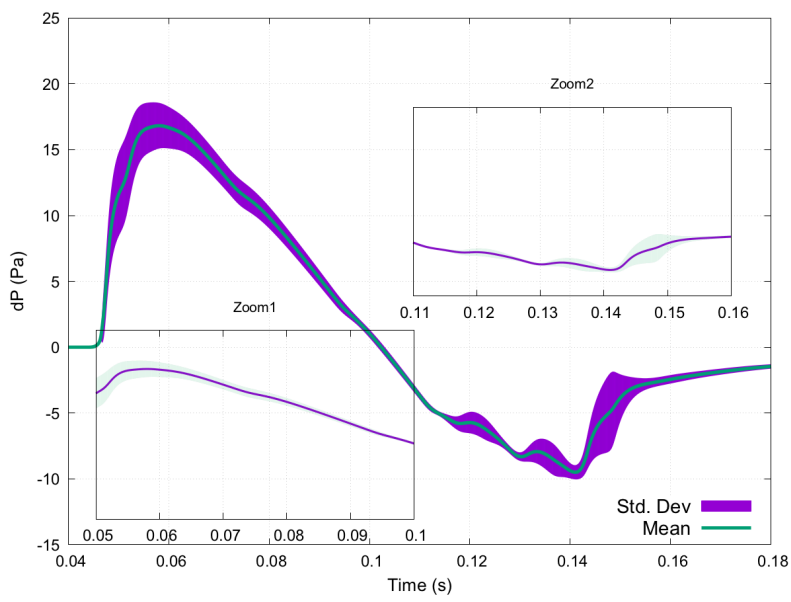

(d) Standard Profile w/ RH=70\% (Optional).

Figure 43. Mean and Standard deviation of Ground Signatures for AXIBODY at Roll Angle $=-45^{\circ}$ 


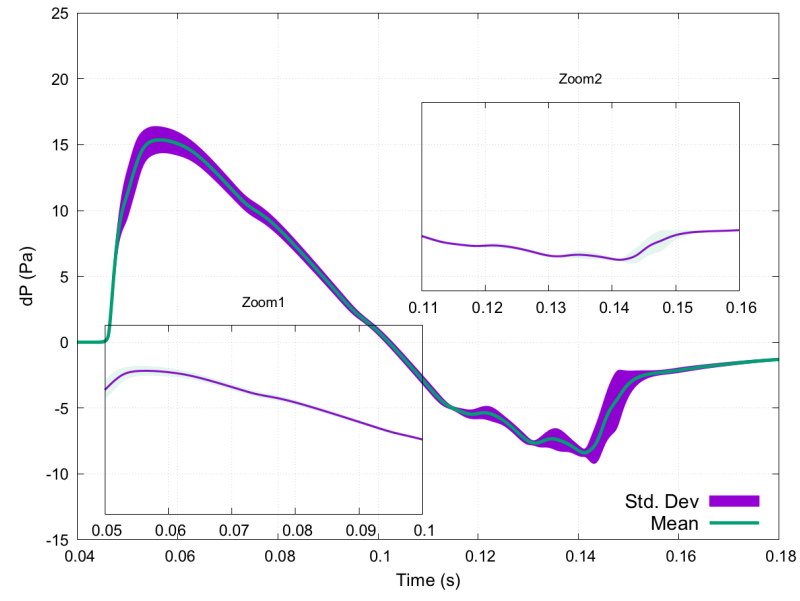

(a) Profile3 (Required).

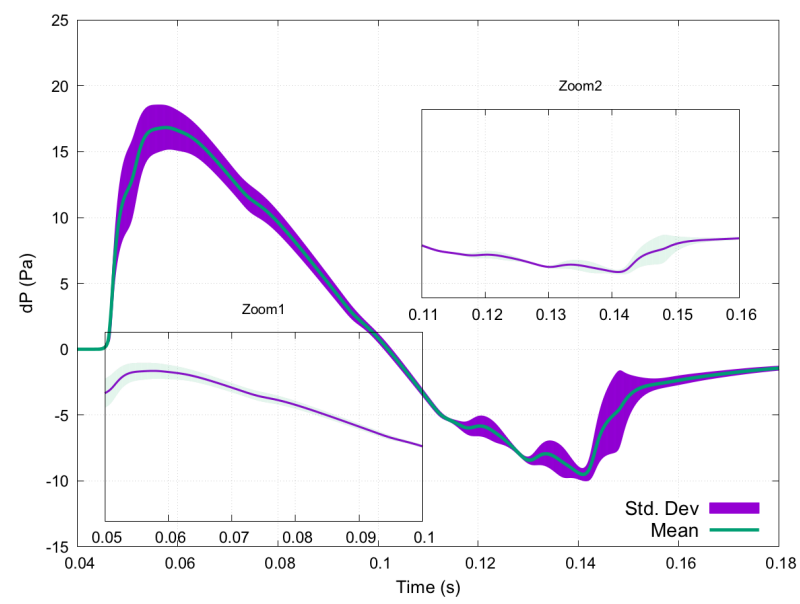

(c) Standard Profile (Optional).

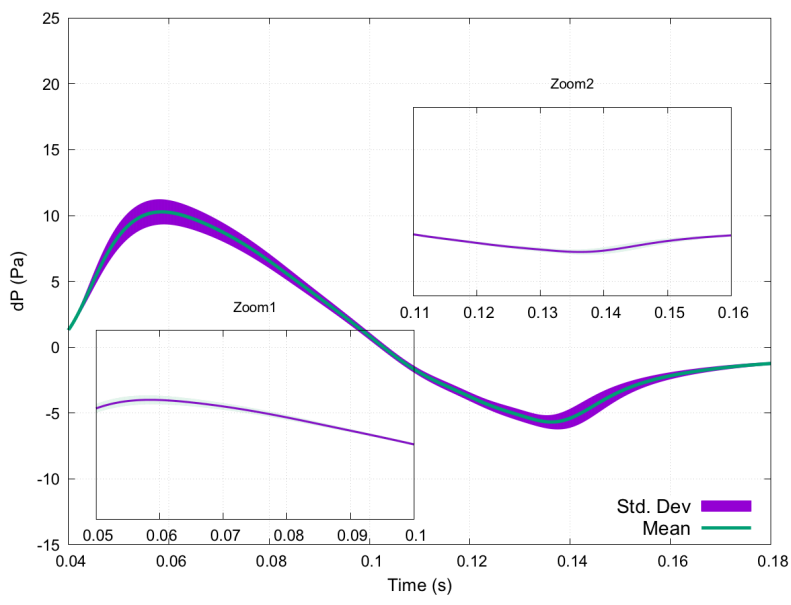

(b) Profile4 (Optional).

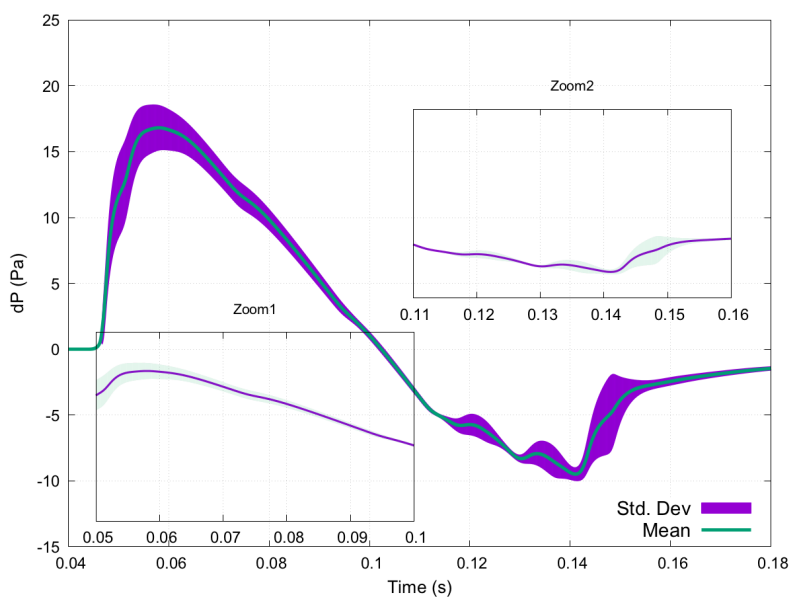

(d) Standard Profile w/ $\mathbf{R H}=\mathbf{7 0 \%}$ (Optional).

Figure 44. Mean and Standard deviation of Ground Signatures for AXIBODY at Roll Angle $=45^{\circ}$ 


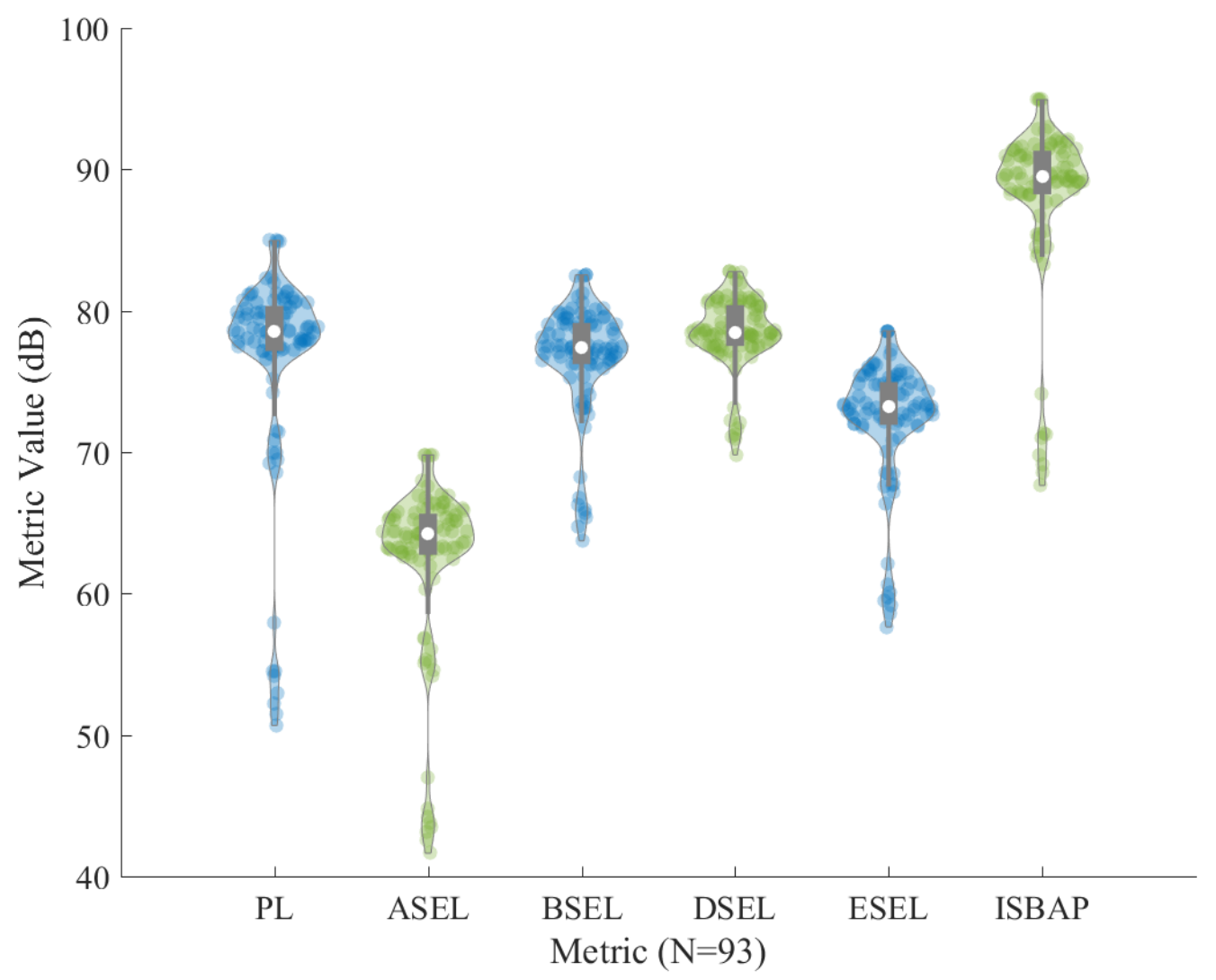

Figure 45. Violin plots of noise metrics for all AXIBODY cases grouped together. 


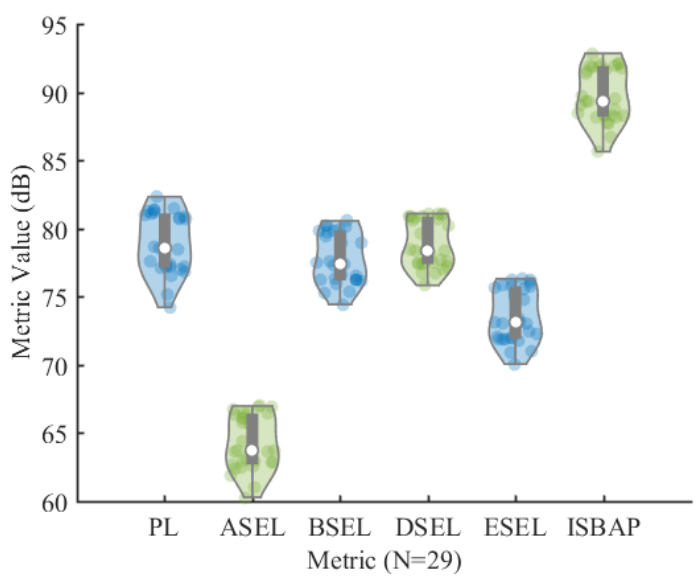

(a) Profile3 (Required).

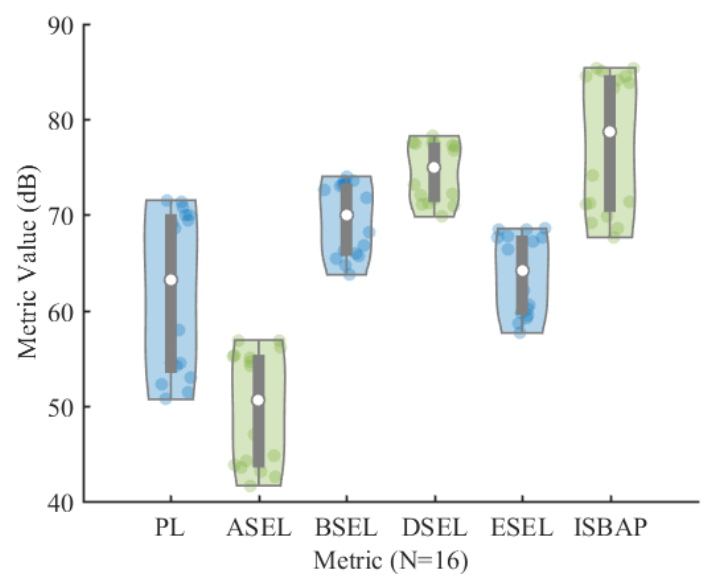

(b) Profile4 (Optional).

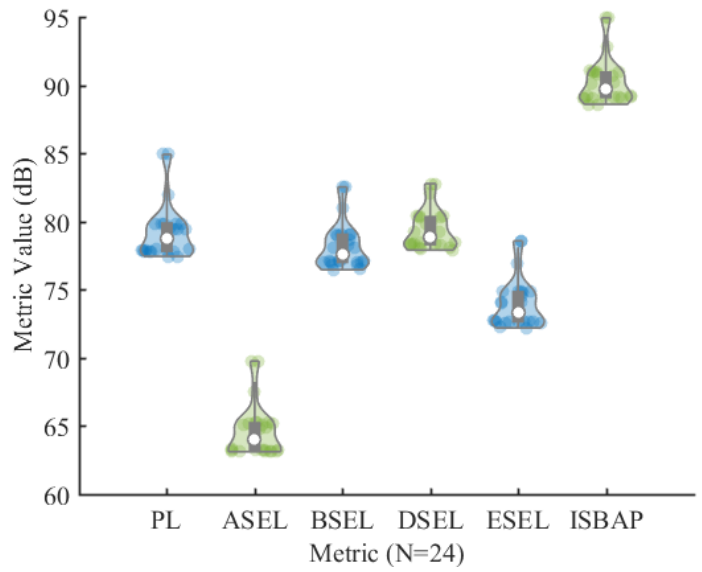

(d) Standard Profile w/ RH=70\% (Optional).

Figure 46. Violin plots of noise metrics for AXIBODY separated by atmospheric conditions (all angles grouped together). 


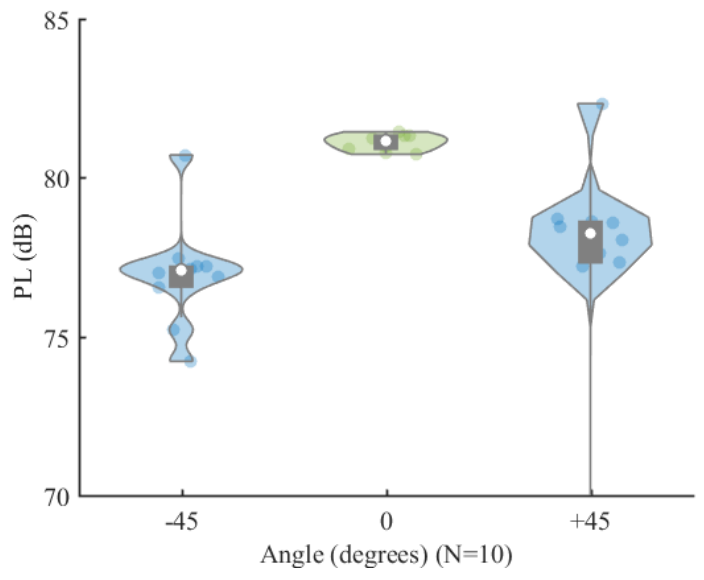

(a) Profile3 (Required).

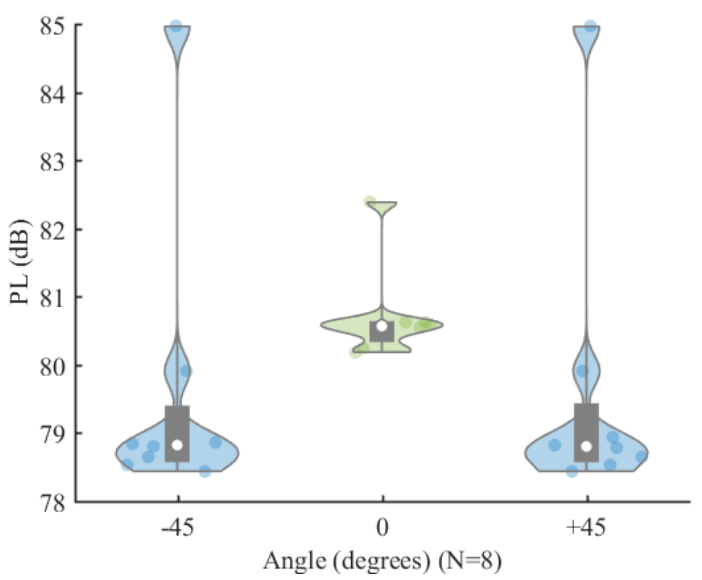

(c) Standard Profile (Optional).

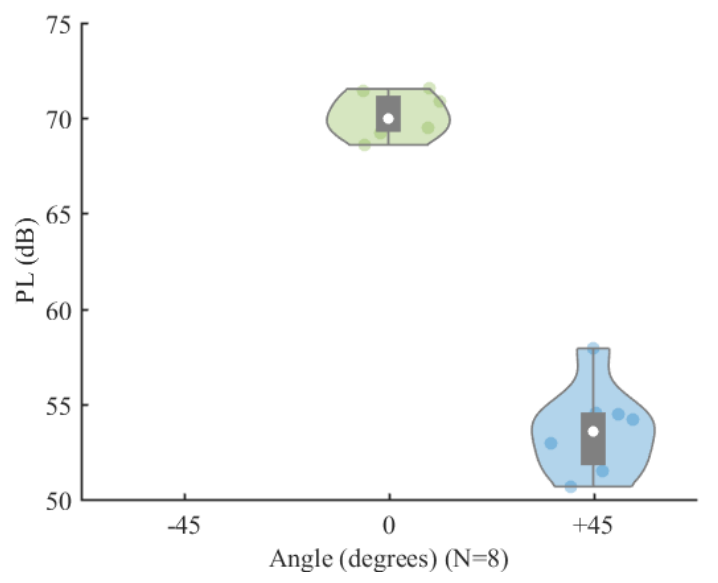

(b) Profile4 (Optional).

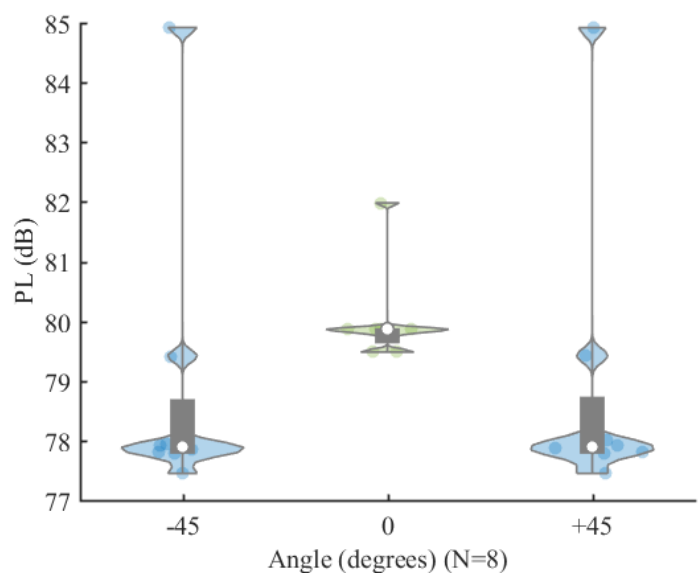

(d) Standard Profile w/ RH=70\% (Optional).

Figure 47. Violin plots of Perceived Level (PL) for AXIBODY separated by atmospheric conditions and by angles. 


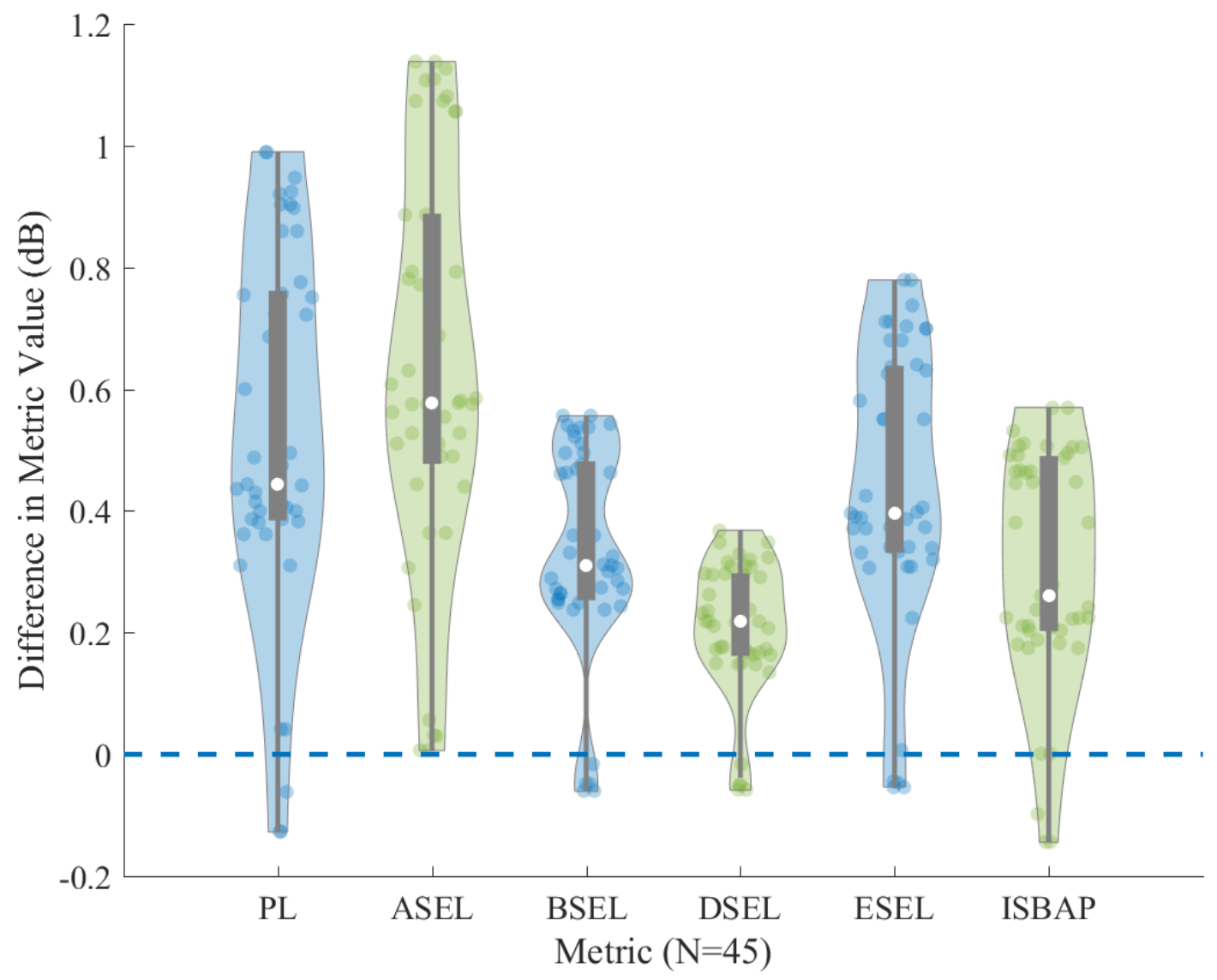

Figure 48. Violin plots of the difference in Perceived Level (PL) between standard atmosphere and standard atmosphere with a relative humidity of $70 \%$ for all AXIBODY and LM1021 angles. 